Florida International University FIU Digital Commons

\title{
Treatment Moderation and Secondary Outcomes: Results from a Randomized Clinical Trial
}

Luci M. Motoca

Florida International University, Imoto001@fiu.edu

DOI: $10.25148 /$ etd.FI12072705

Follow this and additional works at: https://digitalcommons.fiu.edu/etd

\section{Recommended Citation}

Motoca, Luci M., "Treatment Moderation and Secondary Outcomes: Results from a Randomized Clinical Trial" (2012). FIU

Electronic Theses and Dissertations. 669.

https://digitalcommons.fiu.edu/etd/669

This work is brought to you for free and open access by the University Graduate School at FIU Digital Commons. It has been accepted for inclusion in FIU Electronic Theses and Dissertations by an authorized administrator of FIU Digital Commons. For more information, please contact dcc@fiu.edu. 


\section{FLORIDA INTERNATIONAL UNIVERSITY}

Miami, Florida

TREATMENT MODERATION AND SECONDARY OUTCOMES: RESULTS FROM

A RANDOMIZED CLINICAL TRIAL

A dissertation submitted in partial fulfillment of the

requirements for the degree of

DOCTOR OF PHILOSOPHY

in

PSYCHOLOGY

by

Luci M. Motoca

2012 
To: $\quad$ Dean Kenneth G. Furton

College of Arts and Sciences

This dissertation, written by Luci M. Motoca, and entitled Treatment Moderation and Secondary Outcomes: Results from a Randomized Clinical Trial, having been approved in respect to style and intellectual content, is referred to you for judgment.

We have read this dissertation and recommend that it be approved.

William M. Kurtines

\begin{tabular}{rr}
\hline Jeremy Pettit \\
\hline Charles Bleiker
\end{tabular}

Date of Defense: June 28, 2012

Wendy K. Silverman, Major Professor

\begin{tabular}{r} 
Dean Kenneth G. Furton \\
College of Arts and Sciences \\
\hline Dean Lakshmi N. Reddi \\
University Graduate School
\end{tabular}

Florida International University, 2012 


\section{DEDICATION}

I dedicate this Dissertation to my parents, Neculai and Irina, and my sister,

Raluca. Thank you for being so close to me in spirit no matter how far in distance. 


\section{ACKNOWLEDGMENTS}

I would like to thank my committee members Drs. Wendy K. Silverman, William

M. Kurtines, Jeremy Pettit, and Charles Bleiker for helping me conceptualize, write, and analyze the data of my dissertation. I would especially like to thank my major professor and mentor, Dr. Wendy K. Silverman for her support and guidance throughout the entire process and her invaluable feedback. I sincerely respect and admire Dr. Wendy K. Silverman for the dedication that she brings to her work each and every day. I feel incredibly privileged to have had the opportunity to work with and learn from her. I would also like to thank all the graduate students and staff past and present at the Child Anxiety and Phobia Program. I also acknowledge the financial support from the Dissertation Year Fellowship at Florida International University. 
ABSTRACT OF THE DISSERTATION

TREATMENT MODERATION AND SECONDARY OUTCOMES: RESULTS FROM

A RANDOMIZED CLINICAL TRIAL

by

Luci M. Motoca

Florida International University, 2012

Miami, Florida

Professor Wendy K. Silverman, Major Professor

The present study pursued two objectives in the context of a randomized clinical trial of cognitive-behavioral therapy with parent (CBT/P) and group (GCBT) involvement. The first objective was to examine the variability in treatment outcome. There were three specific aims within the first objective, to evaluate: (1) youth characteristics (age, depressive, and externalizing disorders) as moderators of treatment outcome; (2) the differential outcome of the treatment approaches as a function of youth characteristics; and (3) the relative efficacy of the treatment approaches at each level of the moderators.

The second objective was to evaluate the efficacy of anxiety treatments along secondary depressive symptoms and externalizing behaviors. There were five specific aims within the second objective, to evaluate: (1) whether anxiety treatment yields reductions in secondary problems, (2) the efficacy of anxiety treatments in reducing secondary problems as a function of approach and youth characteristics, (3) whether reductions in anxiety symptoms significantly mediate changes in secondary problems, (4) the directionality of change in the hypothesized mediated relations, and (5) whether the 
hypothesized mediated relations are moderated by treatment approach and youth characteristics. The specific aims were pursued using data collected from 183 youth and their mothers. Research questions were tested using multiple regressions and structural equation modeling.

Age, depressive, and externalizing disorders were significant moderators. CBT/P relative to GCBT lowered anxiety more for younger than older youth. GCBT relative to $\mathrm{CBT} / \mathrm{P}$ lowered anxiety more for older than younger youth. GCBT relative to $\mathrm{CBT} / \mathrm{P}$ lowered anxiety more for depressed youth than non-depressed youth. GCBT relative to $\mathrm{CBT} / \mathrm{P}$ lowered anxiety less for externalizing youth than non-externalizing youth. Treatment reduced depressive symptoms and externalizing problem behaviors. Reductions in anxiety mediated changes in depressive symptoms and externalizing problem behaviors. Reversed directionality was found in the relation between social anxiety and depressive symptoms. In CBT/P the direction of change was from depressive to social anxiety. The opposite was true in GCBT. Reductions in social anxiety mediated posttreatment changes in depressive symptoms in GCBT but not CBT/P. The reverse was true at follow-up. Reductions in social anxiety mediated changes in depressive symptoms for girls but not boys. 


\section{TABLE OF CONTENTS}

CHAPTER

PAGE

I. INTRODUCTION

Cognitive Behavioral Treatment for Reducing Anxiety Disorders

Moderators of Treatment Outcome

Secondary Treatment Outcomes

Objectives and Specific Aims of the Present Study

Organization of the Present Study

II. LITERATURE REVIEW

Overview of Treatment Outcome Studies

Treatment Efficacy Studies

Treatment Variability Studies

Examining Moderators: The Exception Rather than the Rule 13

$\begin{array}{ll}\text { Youth Variables as Moderators of Treatment Outcome } & 14\end{array}$

$\begin{array}{ll}\text { Youth Age } & 14\end{array}$

Youth Social Anxiety 16

Parent Variables as Moderators of Treatment Outcome 18

$\begin{array}{ll}\text { Parental Anxiety } & 18\end{array}$

Summary of Studies on Treatment Moderators $\quad 20$

Methodological Issues in Examining for Treatment Moderators 23

Treatment Moderators in the Present Study 25

$\begin{array}{ll}\text { Studies on Secondary Treatment Outcomes } & 30\end{array}$

$\begin{array}{ll}\text { Depressive Symptoms } & 30\end{array}$

Reductions in Depressive Symptoms: ICBT 30

Reductions in Depressive Symptoms: ICBT vs. CBT/P 31

Reductions in Depressive Symptoms: ICBT vs. GCBT 32

Externalizing Problem Behaviors $\quad 32$

Reductions in Externalizing Problem Behaviors: ICBT 33

Reductions in Externalizing Problem Behaviors: ICBT vs. CBT/P 33

Reductions in Externalizing Problem Behaviors: GCBT 35

Summary of Studies on Secondary Treatment Outcomes 35

Secondary Outcomes in the Present Study 35

$\begin{array}{ll}\text { The Present Study } & 44\end{array}$

$\begin{array}{ll}\text { III. METHODOLOGY } & 49\end{array}$

Participants $\quad 49$

$\begin{array}{lr}\text { Measures } & 50\end{array}$

$\begin{array}{ll}\text { Procedures } & 53\end{array}$

Treatment Approaches and Therapists $\quad 53$

IV. RESULTS 56 
V. DISCUSSION 86

Summary of Dissertation Findings $\quad 86$

Contributions and Implications $\quad 90$

Limitations and Directions for Future Research 107

$\begin{array}{ll}\text { LIST OF REFERENCES } & 110\end{array}$

$\begin{array}{ll}\text { APPENDICES } & 121\end{array}$

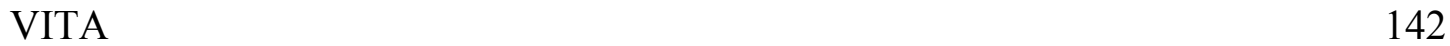




\section{LIST OF TABLES}

TABLES

PAGE

1. Single Degree of Freedom and Interaction Contrasts: RCMAS

122

2. Single Degree of Freedom and Interaction Contrasts: RCMAS/P

123

3. Cell mean anxiety residual change scores: Pre to Post

124

4. Cell mean anxiety residual change scores: Post to Follow-up

125

5. Single Degree of Freedom Contrasts: Secondary Outcomes

126

6. Means for Variables at Pre, Post and Follow-Up

127

7. Fit Indices for Structural Equation Modeling

128 


\section{LIST OF FIGURES}

FIGURES

PAGE

1. Conceptual Model

2. Mediation: Anxiety and Depressive Symptoms

3. Mediation: Anxiety and Depressive Symptoms with Reversed Paths

4. Moderated Mediation: Anxiety and Depressive Symptoms

5. Mediation: Social Anxiety and Depressive Symptoms

6. Mediation: Social Anxiety and Depressive Symptoms with Reversed Paths

7. Moderated Mediation by Treatment: Social Anxiety and Depressive Symptoms

8. Moderated Mediation by Sex and Age: Social Anxiety and Depressive Symptoms

9. Mediation: Anxiety and Externalizing Behaviors

10. Mediation: Anxiety and Externalizing Behaviors with Reversed Paths

11. Moderated Mediation: Anxiety Symptoms and Externalizing Behaviors

12. Treatment Moderation by Depressive Disorders

13. Treatment Moderation by Externalizing Disorders 


\section{CHAPTER I}

\section{INTRODUCTION}

Anxiety disorders in children and adolescents (hereon referred to as youth) are serious, multifaceted problems, which impact not only youth, but also their families and the broader society. Epidemiological studies have shown that anxiety disorders are the earliest and most common psychiatric problems in young people, with prevalence rates ranging from $7 \%$ to $28 \%$ (see Costello, Egger, Copeland, Erkanli, \& Angold, 2011, for

review). Anxiety disorders are chronic conditions that last into adulthood and have low recovery rates (e.g., Last, Perrin, Hersen, \& Kazdin, 1996; Woodward \& Fergusson, 2001). Left untreated, anxiety disorders develop into other psychological disorders, including other anxiety and depressive disorders (e.g., Goodwin, Fergusson, \& Horwood, 2004; Pine, Cohen, Gurley, Brook, \& Ma, 1998). Anxiety disorders in youth are also associated with difficulties in family functioning, peer relationships, and school adjustment (e.g., Ginsburg, La Greca, \& Silverman, 1998; Motoca, Williams, \& Silverman, 2012; Strauss, Frame, \& Forehand, 1987).

\section{Cognitive Behavioral Treatment for Reducing Anxiety Disorders}

Given the prevalence, chronicity, and deleterious outcomes of anxiety disorders in youth, a number of well-designed and well-executed randomized clinical trials (RCTs) have been conducted aimed at reducing these problems (e.g., see Silverman \& Motoca, 2011; Silverman, Pina, \& Viswesvaran, 2008; for reviews). These RCTs have provided strong and consistent empirical evidence for the efficacy of cognitive behavioral treatment (CBT) for youth anxiety and its disorders. Evidence of efficacy is available based on indices of primary outcome (i.e., reductions in anxiety symptoms and diagnostic 
recovery rates for the targeted anxiety disorder) and has been demonstrated in several approaches (e.g., individual, group, parent involvement). Despite research supporting the efficacy of CBT for reducing anxiety and its disorders in youth, two important unresolved issues remain.

\section{Moderators of Treatment Outcome}

The first important unresolved issue is that CBT for anxiety and its disorders is not effective across the board: upward to $50 \%$ of the treated youth in some studies continue to meet diagnostic criteria for their targeted anxiety disorder and remain symptomatic after CBT (e.g., Ginsburg et al., 2011; Silverman et al., 2008). After two decades of treatment research aimed at reducing youth anxiety and its disorders, why do researchers continue to observe such variability in treatment outcome? Perhaps one of the main reasons is because youth with anxiety disorders are heterogeneous in nature. Youth with anxiety disorders often differ in terms of sociodemographic (e.g., age) and clinical (e.g., comorbidity with depressive and externalizing problems) characteristics (e.g., Kendall et al., 2010). It is therefore possible that treatment efficacy varies among subgroups of youth who differ on these and other variables.

One approach used to examine the extent to which efficacy varies among subgroups of treated youth involves testing for moderators of treatment outcome. Moderators are variables that influence the strength of the relation between treatment and outcome to identify subgroups of youth with particularly strong or weak treatment outcome (e.g., Hinshaw, 2007; Kazdin, 2007; Kazdin \& Nock, 2003; Kraemer, Wilson, Fairburn, \& Agras, 2002; La Greca, Silverman, \& Lochman, 2009). Examples of moderators include characteristics of the youth, parents and therapists, which interact 
with treatment approach to either strengthen or weaken treatment outcome (e.g., La Greca et al., 2009).

Testing for moderators of treatment outcome is important for theoretical, empirical, and clinical reasons. Theoretically, knowledge of moderators can inform theories about the development and maintenance of psychopathology (Howe, Reiss, \& Yuh, 2002). As noted by Kraemer, "Moderators could identify subpopulations with possibly different course of illness and may also provide unique new and valuable information to guide future restructuring of diagnostic classification” (p. 4, Kraemer et al., 2002).

Empirically, knowledge of moderators could open avenues for future research aimed at unearthing the processes that work in specific subgroups to either strengthen or weaken treatment outcome (e.g., Kazdin, 2001). Testing for moderators therefore is not a be-all and end-all undertaking but one that serves as a springboard to examine why and how the moderators operate the way they do in future work (e.g., Kraemer et al., 2002). In addition, empirical research on moderators can be used to improve statistical power in future RCTs in that it will lead to the selection of the subgroups of youth for whom specific treatments work particularly well (Kraemer et al., 2002). Knowledge of moderators also can prompt searches for innovative treatments for the subgroups of youth for whom specific treatments do not work as well (Kraemer et al., 2002).

Clinically, testing for moderators answers calls in the literature to move beyond determining whether treatment works to examining for whom or under what conditions it works (e.g., Hinshaw, 2007; Kazdin, 2001; Kazdin, 2007; Kazdin \& Nock, 2003;

Kraemer, Wilson, Fairburn, \& Agras, 2002; La Greca et al., 2009). Testing for 
moderators of treatment outcome is also consistent with the third strategic objective put forth by the National Institute of Mental Health Strategic Plan -- "to develop new and better interventions for mental disorders that incorporate the diverse needs and circumstances of people with mental illness" (NIMH; U.S. Department of Health and Human Services, 2008). The NIMH, in other words, calls for personalized approaches that are tailored to the specific needs of each individual.

Although important for the above reasons, testing for moderators has been an exception not a rule. The reason for this is because traditional intervention research has focused on comparing how groups of individuals receiving an experimental intervention fare against a comparison group that does not receive the intervention (e.g., Kraemer et al., 2002; NIMH; U.S. Department of Health and Human Services, 2008). This approach has yielded information about which treatments are efficacious overall but not about how to choose the best treatment for the specific needs of individuals (Kraemer et al., 2002; NIMH; U.S. Department of Health and Human Services, 2008). Clinical trials that gather a multitude of data and use these data to test for moderators are posed to answer questions that can inform personalized decision making in treatment interventions (NIMH; U.S. Department of Health and Human Services, 2008). Only a small number of studies have tested for treatment moderators (see Chapter 2, Literature Review for further details). Although these initial treatment moderation studies represent valuable efforts, significant gaps and limitations in this research remain, as discussed in this dissertation.

\section{Secondary Treatment Outcomes}

The second important unresolved issue pertains to the assessment of treatment efficacy for anxiety and its disorders along secondary or nontargeted outcomes. The 
impetus to examine the efficacy of CBT for anxiety and its disorders in terms of reductions in depressive symptoms and externalizing problem behaviors, in particular, stems from knowledge that anxiety, depressive and externalizing problems co-occur (e.g., Angold, Costello, \& Erkanli, 1999; Brady \& Kendall, 1992; Costello, Mustillo, Erkanli, Keeler, \& Angold, 2003). The impetus also stems from knowledge that youth with comorbid anxiety and depressive symptoms, as well as youth with comorbid anxiety symptoms and externalizing problem behaviors, experience more severe psychosocial problems than youth with pure anxiety symptoms both concurrently and sequentially (e.g., Franco, Saavedra, \& Silverman, 2007; Strauss, Last, Hersen, \& Kazdin, 1988). To examine a broad range of anxiety treatment efficacy, it is therefore important to assess secondary outcomes in addition to primary anxiety outcomes.

Evaluation of treatment efficacy along secondary outcomes also is important for theoretical, empirical, and clinical reasons (e.g., LoCastro et al., 2009). Theoretically, evaluation of treatment efficacy along secondary outcomes may inform classification efforts (e.g., Lahey, Applegate, Waldman, Loft, Hankin, \& Rick, 2004; Masten et al., 2005). Classification efforts regarding anxiety, depressive, and externalizing symptoms revolve around lumping versus splitting (e.g., Lahey et al., 2004). Because anxiety, depressive, and externalizing symptoms often co-occur across development there is an ongoing debate about common and distinct features of these problems. Anxiety and depressive symptoms are viewed as manifestations of a broad internalizing dimension that is distinct from an externalizing dimension comprising of attention deficit hyperactivity disorder (ADHD), oppositional and conduct disorders, and substance abuse (e.g., Lahey et al., 2004). Classification efforts also revolve around whether anxiety and 
depressive symptoms are distinct enough in youth to warrant separate disorders (e.g., Brady \& Kendall, 1992).

Empirically, evaluation of treatment efficacy along secondary outcomes may inform researchers about the secondary outcome measures that demonstrate sensitivity to treatment and the utility of including secondary outcome measures in RCTs (e.g., LoCastro et al., 2009). Clinically, evaluation of treatment efficacy along secondary outcomes may broaden the focus of interventions to treating not only the anxiety in the person but also psychological difficulties that co-occur with it (NIMH; U.S. Department of Health and Human Services, 2008). Knowledge gathered from evaluation of treatment efficacy along secondary problems also may inform policy makers about the types of treatment programs that are needed and when they are needed (Hudson, Kendall, Coles, Robin \& Webb, 2002; LoCastro et al., 2009).

Although important for the above reasons, only a small number of RCTs for youth anxiety have assessed secondary outcomes in addition to primary anxiety outcomes (see Chapter 2, Literature Review for further details). These initial studies on secondary outcomes represent valuable initial efforts, yet important questions remain as further discussed in this dissertation.

\section{Objectives and Specific Aims of the Present Study}

The important unresolved issues discussed pertaining to treatment moderation and secondary outcomes map onto the two primary objectives of this dissertation study, which will be examined using data collected within the context of a RCT of CBT with parent (CBT/P) and group (GCBT) approaches for youth anxiety and its disorders. The first study objective is to examine variability in treatment outcome in 12 to 14 week 
CBTs aimed at reducing youth anxiety and its disorders. There are three specific aims for the present study's first objective, to evaluate: (1) youth characteristics as moderators of treatment outcome; (2) the differential outcome of each of the treatment approaches as a function of youth characteristics; and (3) the relative efficacy of the treatment approaches at each level of the hypothesized moderator variables.

The second objective is to examine the efficacy of CBTs aimed at reducing anxiety and its disorders along secondary outcomes (i.e., depressive symptoms and externalizing problem behaviors). There are five specific aims within the second objective, to evaluate: (1) whether anxiety treatment yields reductions in secondary problems, (2) the efficacy of anxiety treatments in reducing secondary problems as a function of approach and youth characteristics, (3) whether reductions in anxiety symptoms significantly mediate changes in secondary problems, (4) the directionality of change in the hypothesized mediated relations, and (5) whether the hypothesized mediated relations and the directionality of change between changes in primary anxiety symptoms and secondary outcome problems are moderated by treatment approach (CBT/P versus GCBT) and youth characteristics (sex and age). The present study pursues these specific aims at posttreatment and 12 month follow-up.

\section{Organization of the Present Dissertation}

This dissertation is organized as follows. The dissertation begins with an overview of CBT outcome studies on anxiety and its disorders in youth. This overview contextualizes the present study's objectives within the broader intervention research literature on youth anxiety and its disorders as it relates to two lines of research: (1) research that seeks to understand the variability in treatment outcome by testing for 
predictors and moderators along indices of primary (i.e., targeted) anxiety problems, and (2) research that examines treatment outcome along indices of secondary (i.e., nontargeted) problems.

The Literature Review section summarizes the studies that are related to these two lines of psychotherapy research. It also highlights gaps in each of these two lines of research and outlines the study's research questions. The next section discusses the dissertation's methodology to address the study's research questions. This is followed by a presentation of the dissertation findings. The final section summarizes the dissertation's findings, its contributions and limitations. 


\section{CHAPTER II}

\section{LITERATURE REVIEW}

\section{Overview of Treatment Outcome Studies}

In the past two decades, a growing body of literature has emerged on the treatment of anxiety and its disorders in youth, with over 30 RCTs (e.g., see Silverman et al., 2008; Silverman \& Motoca, 2011 for reviews). Embedded within this literature are several lines of intervention research that evaluate: (1) efficacious and effective approaches in reducing primary or targeted anxiety problems, (2) predictors and moderators in order to understand the variability in treatment outcome, and (3) efficacious and effective approaches in reducing secondary or non-targeted problems. The Literature Review now turns to an overview of the three lines of intervention research. Emphasis is placed on the lines of intervention research on treatment moderation and secondary outcomes, which are directly relevant to the present study. Treatment Efficacy Studies

The first line of research seeks to design and evaluate treatments for youth anxiety and its disorders. This line of research seeks to answer questions about potentially overall efficacious treatments. The key questions asked are the following: Does treatment work? Is treatment efficacious? The basic design of treatment outcome studies involves the random assignment of participants to the approaches of interest. The core statistical analyses used to examine whether treatment is efficacious focus on the main effect of treatment on primary outcome measures from pretreatment to posttreatment and from posttreatment to follow-up. When the relative efficacy of two or more treatment 
approaches is compared, the key questions become: What treatment approach is most efficacious? What treatment approach works best?

Silverman et al. (2008) summarized the literature on treatment outcome and found most of the treatment studies were methodologically robust based on criteria for efficacious treatments (Chambless \& Hollon, 1998; Nathan \& Gorman, 2002). Several of the CBTs reviewed were classified as "probably efficacious" and "possibly efficacious" according to criteria for efficacious treatments. Silverman et al.'s (2008) review begins with the first RCT by Kendall (1994) in which individual CBT (ICBT) was demonstrated to be superior to a waitlist. ICBT fosters change by teaching youth cognitive skills and behavioral strategies to manage their anxiety.

Next, Barrett, Dadds, and Rapee (1996) replicated Kendall's positive findings for the CBT program, and developed a parent program to investigate the possible added benefit of parent involvement. In addition to teaching youth cognitive skills and behavioral strategies to manage their anxiety, CBT/P (and in some studies CBT with family involvement or $\mathrm{CBT} / \mathrm{F}$ ) approaches foster change by teaching parents reinforcement skills (e.g., Barrett, 1998; Barrett et al., 1996; Kendall, Hudson, Gosch, Flannery-Schroeder, \& Suveg, 2008) and improving parents' behaviors and the parentyouth relationship (e.g., Bodden, Bögels, Nauta, De Hann, Ringrose, Appelboom, Brinkman, \& Appelboom-Gerts, 2008; Silverman, Kurtines, Jaccard, \& Pina, 2009; Wood, Piacentini, Southam-Gerow, Chu, \& Sigman, 2006).

Within this first line of research, several RCTs evaluated the efficacy of GCBT with and without parent involvement (e.g., Beidel, Turner, \& Morris, 2000; FlannerySchroeder \& Kendall, 2000; Hayward, Varardy, Albano, Thienemann, Henderson, \& 
Schatzberg, 2000; Silverman, Kurtines, Ginsburg, Weems, Lumpkin, \& Hicks-

Carmichael, 1999b). In addition to teaching youth cognitive skills and behavioral strategies to manage their anxiety, GCBT approaches foster change by teaching peers reinforcement skills and improving youths' social skills behaviors and the peer-youth relationship (e.g., Barrett, 1998; Beidel et al., 2000; Flannery-Schroeder \& Kendall, 2000; Hayward et al., 2000; Spence et al., 2000).

In terms of treatment efficacy, Silverman et al.'s (2008) review revealed that CBTs in general demonstrated positive outcomes. About $50 \%$ of the treated youth no longer meet diagnostic criteria for their targeted anxiety disorder and report reductions in anxiety symptoms that are robust across youth and parent ratings. Positive outcomes have been demonstrated at posttreatment and generally maintained at follow-up. Positive outcomes were demonstrated when CBTs were delivered to the individual child, to the child and his or her parents (usually mothers), and to the child and his or her peers, with generally no statistically significant differences between treatment approaches. However, upward to $50 \%$ of the treated youth in some studies continue to meet diagnostic criteria for their targeted anxiety disorder and remain symptomatic after CBT (e.g., Ginsburg et al., 2011; Silverman et al., 2008). This knowledge has prompted a second line of research that seeks to understand variability in treatment outcome.

\section{Treatment Variability Studies}

This second line of research seeks to understand variability in treatment outcome focusing on the evaluation of predictors and moderators (e.g., Kraemer et al., 2002). There are both similarities and differences when it comes to predictors and moderators of treatment outcome. Understanding the similarities and differences is important because 
key processes relating to treatment outcome tend to be confused in the field, conceptually and statistically. Such confusion can be consequential for how research findings are interpreted and applied in research and clinical practice (e.g., Jaccard \& Guilamo-Ramos, 2002; Kraemer et al., 2002).

In terms of similarities, both predictors and moderators are variables measured at pretreatment (e.g., Kraemer et al., 2002). In terms of differences, predictors are pretreatment variables that account for outcome for the overall treatment sample or irrespective of the type of approach received. The key question asked is the following: What are the characteristics of youth who (fail to) demonstrate positive treatment outcome? The core statistical analyses used to examine predictors of treatment outcome focus on the main effect of pretreatment variables on primary treatment outcome measures at posttreatment and follow-up (e.g., Jaccard \& Guilamo-Ramos, 2002). Predictors of treatment outcome can be evident, for instance, when high levels of youth depressive symptoms are related to high levels of youth anxiety symptoms after treatment irrespective of the type of approach received (La Greca et al., 2009).

Moderators of treatment outcome are pretreatment variables that influence the relation between the type of treatment and outcome (e.g., Kazdin, 2008; Kramer et al., 2002). The key question asked is: For whom and under what conditions does treatment demonstrate positive outcomes? The core statistical analyses to examine moderators of treatment outcome focus on the interaction effect of treatment approach by pretreatment variables in relation to treatment outcomes (e.g., Jaccard \& Guilamo-Ramos, 2002). A statistically significant interaction effect could be found with or without a main effect of treatment on primary outcome measures. A statistically significant interaction effect 
could therefore be found even in the absence of a statistically significant difference between the treatment approaches (e.g., CBT/P versus GCBT). A statistically significant interaction effect could also be found even in the absence of a statistically significant main effect of pretreatment variables on primary outcome measures. Put differently, not all predictors are moderators. Because predictors of treatment outcome using the present study's dataset have been examined elsewhere (Motoca, 2009), of interest in this dissertation are treatment moderators.

\section{Examining Moderators: The Exception Rather than the Rule}

Reviews of treatment outcome research suggest CBT is the most frequently investigated and most empirically supported treatment for youth anxiety and its disorders (e.g., Silverman et al., 2008). Efficacy studies investigating CBT with anxious youth have produced positive results generally with no statistically significant differences between treatment approaches. An issue that may be important to consider is not only whether a treatment is more efficacious than another, but under what circumstances an approach yields stronger outcomes than another. This question is of interest even when, on average, the treatment approaches do not differ in their relative efficacy.

Given the heterogeneity of the population of youth with anxiety disorders it is important to evaluate: (1) youth characteristics (age, depressive, and externalizing disorders) as moderators of treatment outcome; (2) the differential outcome of the treatment approaches as a function of youth characteristics; and (3) the relative efficacy of the treatment approaches at each level of the moderators. Answers to these questions may help clinicians tailor treatment approaches to the specific needs of individuals. The alternative is a trial and error approach whereby various treatments are attempted on the 
individual child with little theoretical or empirical data to guide whether the treatment would work.

Because youth anxiety RCTs have only been reported since 1994 with publication of Kendall (1994), studies on treatment moderators have been the exception rather than the rule. There is currently only one study that has focused specifically on treatment moderators (Alfano, Pina, Villalta, Beidel, Ammerman, \& Crosby, 2009). A small number of studies have reported on moderators when presenting the main treatment outcomes from the respective RCT (e.g., Bodden et al., 2008; Liber, Van Widenfelt, Utens, Ferdinand, Van der Leeden, \& Van Gastel, 2008; Manassis, Mendlowitz, Scapillato, Avery, Fiksenbaum, \& Freire, 2002). These studies have examined youth (i.e., age, depression, social anxiety) and parent (i.e., anxiety) variables as moderators of treatment outcome. By way of background and to highlight the little work that has been done on moderators of treatment outcome, all of these studies are summarized below. Directly relevant to the present study are studies in which youth sociodemographic (i.e., age) and clinical (i.e., comorbidity) characteristics were tested as potential moderators.

\section{Youth Variables as Moderators of Treatment Outcome}

Youth Age. Treatment studies involving CBT approaches for anxious youth have focused on youth from 7 to 16 years, but only two studies tested youth age as a moderator of treatment outcome (Alfano et al., 2009; Bodden et al., 2008). In a sample of 128 youth (8 to 17 years), Bodden et al. (2008) examined efficacy of CBT $(n=64)$ and CBT with family involvement $(\mathrm{CBT} / \mathrm{F} ; n=64)$. Both parents $(91 \%$ fathers; $98 \%$ mothers $)$ and siblings participated in the study. Bodden et al. (2008) examined whether treatment approach (i.e., CBT vs. CBT/F) would interact with youth age on diagnostic recovery 
rates and anxiety symptoms at posttreatment and 3 month follow-up. The rationale for examining youth age as a moderator was that family factors may be more influential for younger children, who tend to depend on their parents; family factors may be less influential for older children, who tend to seek autonomy from their parents. The hypotheses put forth therefore were that CBT/F would be particularly efficacious for younger children and that CBT would be particularly efficacious for older children. Results indicated that youth age was not a moderator of treatment outcome though perhaps low statistical power, a limitation that applies to other studies and that will be further discussed in this dissertation, may explain the null finding.

Alfano et al., (2009) pooled data from two previously published RCTs (i.e., Beidel, Turner, \& Morris, 2000; Beidel, Turner, Sallee, Ammerman, Crosby, \& Pachak, 2007) to examine moderators of treatment outcome in a sample of 88 youth (ages 7 to 17 years) with a primary diagnosis of social phobia (SOP). Youth were randomized to either Social Effectiveness Training for Children (SET-C; $n=57$, Beidel et al., 2007) or the control condition, Testbusters ( $n=31$; Beidel et al., 2000). SET-C (Beidel, Turner, \& Morris, 1998) is a behavioral approach adapted after a program used for adults with SOP (Turner, Beidel, \& Cooley-Quille, 1997). SET-C fosters change in youth social anxiety through targeting social skills, interpersonal functioning, and participation in social activities (Beidel et al., 2008).

Alfano et al. (2009) examined whether clinical severity ratings (CSRs), social anxiety symptoms, and global impairment interacted with youth age to moderate treatment outcome in SET-C. The CSRs were assessed using the Anxiety Disorders Interview Schedule for Children-Child and Parent Versions (ADIS-C/P; Silverman \& 
Albano, 1996); social anxiety symptoms were assessed using the Social Phobia and Anxiety Inventory for Children (SPAI-C; Beidel, Turner, \& Morris, 1995); global impairment was assessed using the Children's Global Assessment Scale (C-GAS; Shaffer et al., 1983). The hypothesis that older youth age would moderate treatment outcome in SET-C was not supported. Of further note, Alfano et al. (2009) also tested youth depressive symptoms as a moderator of treatment outcome in SET-C. Results indicated that youth depressive symptoms did not moderate treatment outcome in SET-C. Overall, in both Bodden et al., (2008) and Alfano et al. (2009), youth age was not a significant moderator of treatment outcome. Both Bodden et al., (2008) and Alfano et al. (2009) did not test for moderators at 12 month follow-up, a limitation that applies to other studies on treatment moderators and that will be further discussed in this dissertation.

Youth Social Anxiety. Two past studies tested for social anxiety as a moderator of treatment outcome in comparative trials of the relative efficacy of ICBT and GCBT (Liber et al., 2008; Manassis et al., 2002). The rationale for testing for social anxiety as a moderator was that the group approach would afford additional social exposures for socially anxious youth and therefore more opportunities for social desensitization than the individual approach. The hypothesis put forth therefore was that children with high levels of social anxiety (SA) and children with diagnosable SOP would improve more in GCBT relative to ICBT.

Manassis et al. (2002) examined the relative efficacy of ICBT $(n=41)$ and GCBT $(n=47)$ in reducing anxiety symptoms in children (ages 8 to 12 years). Manassis et al. (2002) dichotomized their sample into children with low and high levels of SA symptoms based on the Social Anxiety Scale for Children (SASC; La Greca \& Stone, 1993). 
Manassis et al. (2002) tested the interaction between treatment approach and low versus high levels of SA symptoms on treatment outcome. Results indicated that levels of SA symptoms moderated treatment outcome based on children's ratings of anxiety symptoms on the Multidimensional Anxiety Scale for Children (MASC; March, Parker, Sullivan, Stallings, \& Conners, 1997). The results were in the opposite direction than expected. There were greater child rated reductions in anxiety symptoms in ICBT relative to GCBT among children with high levels of SA symptoms. The levels of SA symptoms did not moderate treatment outcome based on mothers' and fathers' ratings of their child's anxiety symptoms on the MASC. Manassis et al. (2002) suggested the group approach may have been overwhelming for socially anxious children making them less likely to fully benefit from treatment.

Liber et al. (2008) examined the relative efficacy of ICBT $(n=41)$ and GCBT $(n$ $=47$ ) in reducing anxiety symptoms in children (ages 8 to 12 years). Liber et al. (2008) tested the interaction between type of treatment and diagnosable SOP on treatment outcome. Results indicated that SOP moderated treatment outcome based on fathers' ratings of their child's anxiety symptoms on the Internalizing broadband scale of the Child Behavior Checklist (CBCL-I; Achenbach \& Rescorla, 2001). GCBT relative to ICBT lowered anxiety symptoms significantly more for children with SOP than children without SOP. This interaction effect was not found based on children's self-ratings of anxiety symptoms on the MASC, nor based on mothers' ratings of their child's anxiety symptoms on the CBCL-I.

Overall, Manassis et al. (2002) and Liber et al. (2008) produced inconsistent findings. ICBT relative to GCBT lowered anxiety symptoms significantly more for 
children with high levels of SA symptoms in Manassis et al. (2002). GCBT relative to ICBT lowered anxiety symptoms significantly more for children with SOP than for children without SOP in Liber et al. (2008). Both sets of findings were not robust across informants. Both Manassis et al. (2002) and Liber et al. (2008) did not test for moderators at 12 month follow-up.

\section{Parent Variables as Moderators of Treatment Outcome}

Parental Anxiety. Two past studies tested for parental anxiety as a moderator of treatment outcome in studies comparing the relative efficacy of ICBT and CBT/F or CBT/P (Bodden et al., 2008; Kendall et al., 2008). In both studies parental anxiety was assessed using the Anxiety Disorder Interview Schedule for DSM-IV, Lifetime Version (ADIS-IV-L; DiNardo, Brown, \& Barlow, 1994). Of interest was the interaction between treatment approach and parental diagnostic status (i.e., mothers with anxiety disorders versus mothers without anxiety disorders; fathers with anxiety disorders versus fathers without anxiety disorders; both parents with anxiety disorders versus both parents without anxiety disorders). Youth whose parents suffered from anxiety disorders were expected to improve more in $\mathrm{CBT} / \mathrm{F}$ or $\mathrm{CBT} / \mathrm{P}$ than ICBT.

Bodden et al., (2008) examined the relative efficacy of ICBT $(n=64)$ and CBT/F $(n=64)$. Both parents ( $91 \%$ fathers; $98 \%$ mothers) and siblings participated in the study. Bodden et al. (2008) examined whether the treatment approach (ICBT vs. CBT/F) would interact with parental anxiety on diagnostic recovery rates and anxiety symptoms at posttreatment and 3 month follow-up. Measures included the Screen for Child Anxiety Related Emotional Disorders (SCARED; Birmaher, Khetarpal, Brent, Cully, Balach, Kaufman et al., 1997) and the State Trait Anxiety Inventory (STAI; Spielberger, 1973) 
completed by the youth (SCARED-C; STAI-C) and by the parents (SCARED-P; STAIC); the CBCL completed by the parents, and the Children's Automatic Thoughts Scale (CATS; Schniering \& Rapee, 2002) completed by the youth.

Results indicated that parental anxiety was a significant moderator of treatment outcome, however, in the opposite direction than expected and only on one measure. ICBT was particularly efficacious for youth whose parents had anxiety disorders themselves; $\mathrm{CBT} / \mathrm{F}$ was particularly efficacious for youth whose parents did not have anxiety disorders themselves. For youth whose parents had anxiety disorders significantly more fell within the normal range of the SCARED-C after CBT ( $46 \%$ at posttreatment; $75 \%$ at 3 month follow-up) than after CBT/F (19\% at posttreatment; $46 \%$, at 3 month follow-up). For youth whose parents did not have anxiety disorders, significantly more fell within the normal range of the SCARED-C after CBT/F (62\% at post-treatment; $73 \%$ at 3 month follow-up) than after ICBT (55\% at post-treatment; $63 \%$ at 3 month followup). This interaction effect was not found based on the SCARED-P, nor the STAI-C and STAI-P. Bodden et al. (2008) suggested parental anxiety may have blocked the transfer of control of CBT skills from parents to youth in $\mathrm{CBT} / \mathrm{F}$ which may explain why ICBT was particularly efficacious for youth whose parents had anxiety disorders themselves; CBT/F was particularly efficacious for youth whose parents did not have anxiety disorders themselves.(e.g., Ginsburg, Silverman, \& Kurtines, 1995).

Kendall et al. (2008) examined the relative efficacy of ICBT ( $n=55), \mathrm{CBT} / \mathrm{P}(n=$ 56), and education/support/attention with parents $(\mathrm{ESA} / \mathrm{P} ; n=50)$ in a sample of 161 youth (ages 7 to 14 years) diagnosed with an anxiety disorder. Kendall et al. (2008) examined whether the treatment approach (i.e., ICBT, CBT/P, ESA/P) would interact 
with parental anxiety on anxiety symptoms at posttreatment and 12 month follow-up. Measures included the MASC completed by the youth and CBCL-I completed by the parents.

Results indicated that parental anxiety was a significant moderator of treatment outcome from posttreatment to 12 month follow-up. Youth with an anxious father who received ICBT showed significantly less reductions in anxiety symptoms on the MASC from posttreatment to 12 month follow-up than children with an anxious father who received CBT/P. This interaction effect from posttreatment to 12 month follow-up was not found based on parents' ratings of their children's anxiety symptoms on the CBCL-I. Parental anxiety was not a significant moderator of treatment outcome from pretreatment to posttreatment. Analyses also examined the interaction between parent anxiety and treatment approach in relation to child diagnostic outcomes. The results of these analyses, however, were not reported due to small cell sizes although the authors' conclusion was that parental anxiety was not a significant moderator of treatment outcome.

\section{Summary of Studies on Treatment Moderators}

Moderators of treatment outcome were tested in a small number of studies. Two reasons have been put forth as to why moderators of treatment outcome have been infrequently tested (e.g., Silverman \& Motoca, 2011). The first reason is that most treatment studies were conducted primarily to investigate whether CBT is efficacious.

The second reason is that most treatment studies were insufficiently powered to systematically test for moderators (e.g., Kendall et al., 2008). The studies summarized above represent initial valuable efforts toward advancing the knowledge base on 
moderators of treatment outcome; yet three gaps in treatment moderation research remain.

First, prior research on treatment moderation examined youth age and comorbidity, variables directly relevant to the present study, in the context of RCTs comparing the relative efficacy of ICBT versus CBT/P (i.e., Bodden et al., 2008) or SETC versus Testbusters (i.e., Alfano et al., 2009). Moderators of treatment outcome are yet to be examined in the context of RCTs comparing the relative efficacy of $\mathrm{CBT} / \mathrm{P}$ versus GCBT. This is because although there now exists considerable empirical evidence that youth anxiety and its disorders can be reduced in CBT programs that incorporate parents and group contexts and target specific content areas relevant to these contexts, no published RCTs have directly evaluated the relative efficacy of CBT/P and GCBT in the same investigation. The present study was in the unique position to test for moderators in the context of a RCT that directly evaluated the relative efficacy of CBT/P and GCBT in the same investigation (Marin, 2010). Testing for moderators in this way is timely because statistically significant reductions in anxiety symptoms have been demonstrated with both treatment approaches (Marin, 2010). It is now important to examine individual differences in the effects of $\mathrm{CBT} / \mathrm{P}$ and GCBT or for whom these treatment approaches yield the strongest and weakest outcomes (e.g., Kraemer et al., 2002).

Second, prior research on treatment moderation has not paid attention to youth age and comorbidity as moderators at 12 month follow-up. Testing for youth age and comorbidity as moderators at 12 follow-up is important because such analyses would reveal whom and under what conditions maintains their therapeutic change, makes additional gains or deteriorates over a relatively long period of time. Knowledge gathered 
from testing for moderators both at posttreatment and 12 follow-up can illuminate the importance of time and timing in therapeutic change (e.g., Granic, 2006; Silverman et al., 2009).

In pursuing questions about time and timing, for example, some investigators have described lagged effects in the process of therapeutic change (e.g., Jaccard \& Jacoby, 2010; Silverman et al., 2009). Silverman et al. (2009) examined whether youth anxiety between pretreatment and posttreatment, as reported by parent and youth, served as a mediator between parent variables (i.e., parental positive-negative behaviors towards the child, conflict in the parent-youth dyadic relationship, and parental anxiety) measured at posttreatment and parent variables measured at 12 month follow-up. Findings indicated changes in youth anxiety from pretreatment to posttreatment mediated changes in parent anxiety during the same time period. Findings also indicated a lagged effect such that it took time for changes in youth anxiety from pretreatment to posttreatment to yield changes in positive parenting behaviors, measured at the 12 month follow-up. Such lagged effects make theoretical sense because time may need to pass for changes in one variable to work their way through and produce changes in another variable.

Testing for treatment moderators at both posttreatment and 12 month follow-up constitutes another approach to understanding the importance of time and timing in therapeutic change. If an individual characteristic (e.g., presence of depression) interacts with treatment to moderate posttreatment but not 12 month follow-up outcomes, this would suggest that youth who share that characteristic may need additional time to "catch up" in anxiety symptom reduction with youth who do not share that characteristic and this may vary by treatment approach. If an individual characteristic (e.g., presence of 
depression) interacts with treatment to moderate 12 month follow-up but not posttreatment outcomes, this would suggest that youth who share that characteristic may deteriorate over time relative to youth who do share that characteristic and this may vary by treatment approach. Testing for treatment moderators at both posttreatment and 12 month follow-up could therefore identify subgroups of youth with different course of therapeutic change (e.g., Kraemer et al., 2002).

Third, prior research on treatment moderation is limited because most studies relied on traditional data analytic techniques to test for moderators. Traditional data analytic techniques have several caveats when it comes to testing for moderators of treatment outcome (e.g., Jaccard \& Guilamo-Ramos). These caveats are discussed next as part of methodological issues in testing for treatment moderators.

Methodological Issues in Testing for Treatment Moderators

Jaccard and Guilamo-Ramos (2002) discussed that the traditional approach to interpreting a statistically significant interaction effect in a factorial design is to use simple main effects analysis. Jaccard and Guilamo-Ramos (2002) further discussed that simple main effects address the question of whether an independent variable has an effect on a dependent variable at each level of a moderator variable. For example, when testing for youth age as a moderator, researchers might ask if the treatment approaches (e.g., $\mathrm{CBT} / \mathrm{P}, \mathrm{GCBT}$ ) differ in their means when only the younger children in the approach study are considered. The researchers might also ask whether the treatment approaches (e.g., CBT/P, GCBT) differ in their means when only the older children in the approach are considered. These questions may themselves be of interest, but do not effectively address interaction effects. They address simple main effects. 
To effectively address interaction effects, one must conduct single degree of freedom interaction contrasts (Jaccard, 1998; Jaccard \& Guilamo-Ramos, 2002). Interaction effects can be conceptualized in different ways. One common way involves a dependent variable, an independent variable, and a moderator variable (Holmbeck, 1997; Jaccard, 1998; Jaccard \& Guilamo-Ramos, 2002). The independent variable is the assumed cause of the dependent variable. In the case of an interaction, the effect of the independent variable on the dependent variable changes depending on the value of a third variable, the moderator variable.

An interaction contrast compares the effect of an independent variable on a dependent variable in one group with the comparable effect of an independent variable on a dependent variable in another group. For example, an interaction effect compares whether the effect of GCBT relative to $\mathrm{CBT} / \mathrm{P}$ is stronger for older children than it is for younger children. This differential effect as a function of age is at the core of an interaction effect. Single degree of freedom interaction contrasts are conducted by contrasting the main effect of GCBT relative to $\mathrm{CBT} / \mathrm{P}$ for younger children to the main effect of GCBT relative to $\mathrm{CBT} / \mathrm{P}$ for older children. If the single degree of freedom interaction contrast is significant, then one concludes that the interaction is significant (e.g., Jaccard \& Guilamo-Ramos, 2002).

None of the studies reviewed above that have tested for moderators reported single degree of freedom interaction parameters as recommended by Jaccard and Guilamo-Ramos (2002). Instead, interaction analyses were undertaken in some studies using multivariate analyses of variance, which were followed by univariate analyses of variance on each outcome or simple main effects. Jaccard and Guilamo-Ramos (2002) 
cautioned against using simple main effects as tests for moderators because this practice "fails to formally compare effects in the two groups" (p. 139). This dissertation study follows the recommendations by Jaccard and Guilamo-Ramos (2002) to test for treatment moderators.

\section{Treatment Moderators in the Present Study}

The first objective of the present study therefore was to evaluate youth characteristics as moderators of treatment outcome. The youth characteristics of interest were age, comorbidity with depressive disorders and comorbidity with externalizing disorders.

Youth Age. Although youth age has not been found as a moderator of treatment outcome, there are theoretical reasons to believe this would be the case when the comparison treatment approaches are CBT/P and GCBT. Two complementary hypotheses are feasible to describe the direction of the interaction. First, CBT/P relative to GCBT may lower anxiety symptoms more for younger than older children. This hypothesis is reasonable because a treatment approaches that aims to improve parenting skills (i.e., CBT/P) may have greater therapeutic significance for younger than older children relative to a treatment approach that aims to improve social skills (e.g., Daleiden, Vasey, \& Brown, 1999; Hudson et al., 2002). CBT/P may have a stronger impact on treatment outcomes for young children because parents play an influential role in the lives of these children. Daleiden et al. (1999), for example, suggested parents play a greater role in arranging exposures for younger than older children. Improving parenting skills may be important for younger children; other processes may bring about anxiety symptom reduction for older children. Older children undergo a process of 
gaining autonomy from their parents. The need for increased autonomy in older children may make parental involvement in treatment a challenging task (e.g., Hudson et al., 2002). Thus, during the developmental period when parents play an influential role in their children's lives, CBT/P relative to GCBT may lower anxiety symptoms more for younger than older children.

Second, GCBT relative to CBT/P may lower anxiety symptoms more for older than younger children. This hypothesis is reasonable because a treatment approach that aims to improve social skills and the peer-youth relationship (i.e., GCBT) may have greater therapeutic significance for older relative to younger children. Difficulties with social skills and peer-youth relationships are most likely to be recognized in adolescence, when children spend many hours with peers and developing peer relationships becomes a central developmental task (e.g., Parker, Rubin, Erath, Wojslawowicz, \& Buskirk, 2006). It is also in adolescence that the social skills involved in effective peer-youth relationships become increasingly complex (e.g., Parker et al., 2006). Thus, during the developmental period when anxious youth are most likely to be identified as having difficulties with social skills and peer-youth difficulties, GCBT relative to CBT/P may lower anxiety symptoms more for older than younger children.

Comorbidity. In terms of comorbidity, a recurrent theme throughout RCTs for youth anxiety and its disorders are the high rates of comorbidity in the study samples, with the most common co-occurring disorders being other anxiety, depressive and externalizing disorders (see Ollendick, Jarrett, Grills-Taquechel, Hovey, \& Wolff, 2008, for a review). Given this high rate of comorbidity, one question that has received attention in recent years is whether the presence of comorbidity hinges on treatment 
outcome. Most of the past research that sought to understand the variability in treatment outcome examined comorbidity as a predictor of treatment outcome (e.g., Berman, Weems, Silverman, \& Kurtines, 2000). Scant research has been conducted on comorbidity, in general, and comorbidity with depressive and externalizing disorders, in particular, as treatment moderators; the present study will begin to fill this gap in knowledge.

Comorbidity with Depressive Disorders. Consideration of comorbidity with depressive disorders as a moderator of treatment outcome is important because these problems affect the internal, psychological environment of the child and may hinder outcome in some CBTs but not others. GCBT relative to CBT/P may lower anxiety symptoms more for youth with comorbid depressive disorders than youth without comorbid depressive disorders. This hypothesis is feasible because a treatment approach that aims to improve social skills and the peer-youth relationship (i.e., GCBT) may have greater therapeutic significance for youth with comorbid depressive disorders than youth without comorbid depressive problems. Depressed youth are often described as having social skills deficits, which are hypothesized to maintain depressive symptoms (Lewinsohn, 1974).

Depression also disrupts peer-youth relationships by evoking negative responses from others and generating interpersonal stress and conflict (Joiner, Coyner, \& Blalock, 1999). Depressed youth rate themselves as less able to resolve conflict and less able to provide social support to peers relative to non-depressed youth (e.g., Hammen, Shih, \& Brennan, 2003). When compared to CBT/P, a treatment approach that targets difficulties with social skills and peer-youth relationships (i.e., GCBT) may lower anxiety symptoms 
more for youth with comorbid depressive disorders than youth without comorbid depressive disorders. For the purposes of the moderation analyses, depressive disorders were operationally defined in this study by the presence of an affective disorder in youth's diagnostic profile as assessed by the ADIS: C/P (Silverman \& Albano, 1996).

Comorbidity with Externalizing Disorders. Consideration of comorbidity with externalizing disorders as a moderator of treatment outcome is also important because these problems present themselves outwardly and involve youth acting negatively towards others (Campbell, Shaw, \& Gilliom, 2000; Hinshaw, 1987). Comorbid externalizing disorders may hinder treatment from being effective due to implementation difficulties in some CBTs but not others (Campbell et al., 2000; Hinshaw, 1987). Most CBTs designed for anxiety and its disorders have as a core component exposure to the feared object, people, or events (Silverman, et al., 1999a). However, the CBTs differ in the extent to which the child's parents or the child himself or herself control the out of session exposure (Silverman, et al., 1999a, b).

CBT/P for example equips parents with skills to set appropriate contingencies for successful child non-avoidance. CBT/P therefore places parents in control of the child's out of session exposure and approach behavior. In the absence of parent training, GCBT relies on the child's own abilities to regulate emotions when it comes to out of session exposure and approach behavior. GCBT therefore places the child in relatively greater control of his or her out of session exposure and approach behavior than CBT/P. Given that GCBT is relatively more reliant upon the child's own abilities to regulate emotions that youth with comorbid externalizing disorders may not necessarily have, it is reasonable that GCBT relative to $\mathrm{CBT} / \mathrm{P}$ may lower anxiety symptoms less for youth 
with comorbid externalizing disorders than youth without comorbid externalizing disorders.

The transfer of control model (Silverman \& Kurtines, 1996) guided this hypothesis. Transfer of control refers to the treatment goal of transferring the skills for improving target behaviors from the therapist to the child through a mediator. According to the transfer of control model, in approaches that involve the parents (e.g., CBT/P), the parents represent the specific mediator of treatment outcome in that a transfer of control occurs from the therapist to the child through the parents. In approaches that involve the peers (i.e., GCBT), the peers represent the specific mediator of treatment outcome in that a transfer of control occurs from the therapist to the child through the peers in the therapy group.

There may be a block in the transfer of control from peers to youth in GCBT due to children's inability to regulate or control their own externalizing problem behaviors. In addition, GCBT does not equip parents with skills to set appropriate contingencies to successfully manage their child's non-avoidance and other behaviors such as externalizing problems. Comorbidity with externalizing disorders may therefore pose a problem for treatment outcome in GCBT. A parallel block in the transfer of control may not occur in CBT/P because parents may use skills to set appropriate contingencies to successfully manage not only their child's non-avoidance but also other behaviors such as externalizing problem behaviors. Comorbidity with externalizing disorders may therefore not pose a problem for treatment outcome in CBT/P. For the purposes of the moderation analyses, the present study relied on the presence of an externalizing disorder in youth's diagnostic profile as assessed by the ADIS: C/P (Silverman \& Albano, 1996). 


\section{Studies on Secondary Treatment Outcomes}

As noted at the beginning of this chapter, the third line of approach research seeks to evaluate the efficacy of anxiety treatments along secondary or non-targeted problems, including depressive symptoms and externalizing problem behaviors. Historically, treatment outcome studies have focused on the efficacy of anxiety treatments along secondary or non-targeted problems, including anxiety symptoms and disorders. There is currently only one study that has focused specifically on secondary outcomes (Suveg, Hudson, Brewer, Flannery-Schroeder, Gosch, \& Kendall, 2009). A small number of studies have reported on secondary outcomes when presenting the main treatment outcomes from the respective RCT (e.g., Barrett et al., 1996; Kendall, 1994; Kendall, Flannery-Schroeder, Panichelli-Mindel, Southam-Gerow, Henin, \& Warman, 1997; Manassis et al., 2002; Nauta, Schooling, Emmelkamp, \& Minderaa, 2003; Silverman, Kurtines, Ginsburg, Weems, Lumpkin, \& Hicks-Carmichael, 1999a).

Depressive Symptoms. Several studies have examined treatment outcome along depressive symptoms in ICBT, CBT/P, and GCBT (Barrett et al., 1996; Kendall, 1994; Kendall et al., 1997; Manassis et al., 2002; Nauta et al., 2003; Silverman et al., 1999a; Suveg et al., 2009). In all of these studies depressive symptoms were assessed using the Children's Depression Inventory (CDI; Kovacs, 1981). These studies are summarized next in the following order: ICBT, CBT/P and GCBT.

Reductions in Depressive Symptoms: ICBT. Silverman et al. (1999a) examined the effects of exposure plus contingency management $(n=40)$, exposure plus cognitive selfcontrol $(n=41)$, and an education-support control approach $(n=23)$ on depressive symptoms in youth (6 to 16 years) who were treated for simple phobias. Similar 
significant reductions in depressive symptoms from pretreatment to posttreatment were been found by Kendall in two studies (Kendall, 1994; $N=47$ youth; 9 to 13 years; Kendall et al., 1997) in which CBT was compared to a waitlist control approach. In both of these studies, significant reductions in depressive symptoms among youth who received CBT were maintained at 12-month follow-up.

Reductions in Depressive Symptoms: ICBT vs. CBT/P. Barrett et al. (1996) examined the effects of ICBT $(n=28)$ and CBT/P $(n=25)$ on depressive symptoms in youth (6 to 16 years) treated for anxiety disorders. Significant reductions in depressive symptoms were found from pretreatment to post-treatment. Further reductions in depressive symptoms were found at 6- and 12-month follow-up. Nauta et al. (2003) similarly examined the effects of CBT $(n=29)$ and CBT/P $(n=30)$ on depressive symptoms in youth (7 to 18 years) treated for anxiety disorders. Results revealed significant reductions in depressive symptoms from pretreatment to posttreatment with no statistically significant differences between treatment approaches. The effects of treatment on depressive symptoms were maintained at 3-month follow-up.

Suveg et al. (2009) used data from the RCT by Kendall et al. (2008) to examine the generalizability of positive treatment effects from the targeted, anxiety symptoms to secondary, nontargeted depressive symptoms. Participants were 161 anxious youth (7 to 14 years) who were randomly assigned to $\mathrm{CBT}(n=55), \mathrm{CBT} / \mathrm{P}(n=56)$, and ESA/P $(n=$ 50). Results revealed significant reductions in depressive symptoms from pretreatment to posttreatment with no statistically significant differences between treatment approaches. Reductions in depressive symptoms were maintained at 12 month follow-up. There were no statistically significant differences in reductions in depressive symptoms based on 
youth sex and age. There was not a statistically significant interaction between treatment approach and youth sex and age, respectively on reductions in depressive symptoms.

Reductions in Depressive Symptoms: ICBT vs. GCBT. Manassis et al. (2002) examined the effects of ICBT $(n=41)$ and GCBT $(n=47)$ on depressive symptoms in children (ages 8 to 12 years) who were treated for anxiety disorders. Results revealed significant reductions in depressive symptoms from pretreatment to posttreatment. There were statistically significant greater reductions in depressive symptoms in ICBT relative to GCBT. The reason for the superior performance of CBT on depressive symptoms is unclear. Manassis et al. (2002) suggested the group approach may have been overwhelming for children with social evaluative concerns. The individual treatment approach may have offered children more direct reassurance and social approval by the therapist, which may work particularly well with anxious children. These possibilities may be the reasons why greater reductions in depressive symptoms were unexpectedly found in ICBT relative to GCBT. These possibilities highlight the need for further research on determining whether specific symptoms are more amenable to one treatment approach versus another. Follow-up data were not reported.

Liber et al. (2008) examined the effects of ICBT $(n=65)$ and GCBT $(n=62)$ on depressive symptoms in youth (ages 8 to 12 years) who were treated for anxiety disorders. Results revealed significant reductions in depressive symptoms from pretreatment to posttreatment with no statistically significant differences between treatment approaches. Follow-up data were not reported.

Externalizing Problem Behaviors. Several studies have examined treatment outcome along externalizing problem behaviors in ICBT, CBT/P, and GCBT with and 
without parent involvement (Barrett, 1998; Kendall, 1994; Nauta et al., 2003; Pina, Silverman, Fuentes, Kurtines, \& Weems, 2003; Suveg et al., 2009). In all of these studies externalizing problem behaviors were assessed using the Externalizing broadband scale of the Child Behavior Checklist (CBCL-E; Achenbach \& Rescorla, 2001). These studies are summarized next in the following order: ICBT, CBT/P and GCBT. Reductions in Externalizing Symptoms: ICBT

Kendall (1994) reported significant reductions in externalizing problem behaviors at posttreatment and 12 month follow-up among youth $(\mathrm{N}=47$; ages 9 to 13 years $)$ who received ICBT. Similarly, significant reductions in externalizing symptoms were reported by Pina et al. (2003) who examined CBT for European American and Hispanic Latino youth. Both European American and Hispanic Latino youth demonstrated significant reductions in externalizing problem behaviors at posttreatment. Significant reductions in externalizing problem behaviors were maintained at 3, 6, and 12 month follow-up with no statistically significant differences between American and Hispanic Latino youth. Reductions in Externalizing Symptoms: ICBT vs. CBT/P

In the study reported above by Suveg et al. (2009), which used data from the RCT by Kendall et al. (2008) to examine the relative efficacy of ICBT and CBT/P along externalizing problem behaviors. Results revealed significant reductions in externalizing problem behaviors from pretreatment to posttreatment on mothers' and fathers' CBCL-E ratings with no statistically significant differences between treatment approaches. Further reductions in externalizing problem behaviors were found at 12 month follow-up again with no statistically significant differences between treatments. 
There were no statistically significant differences based on youth sex at posttreatment and 12 month follow-up on parents' CBCL-E ratings. There was a statistically significant difference based on youth age at posttreatment on fathers' but not mothers' CBCL-E ratings. Younger compared to older children showed significant reductions in father-rated externalizing from pretreatment to posttreatment. There was no statistically significant interaction between treatment and youth sex and age, respectively on reductions in externalizing problem behaviors.

Nauta et al. (2003) also examined the relative efficacy of ICBT and CBT/P along externalizing problem behaviors. Unlike Suveg et al. (2009), who found significant reductions in externalizing problem behaviors when the treatment approaches of interest were ICBT and CBT/P, Nauta et al. found externalizing problem behaviors remained unchanged in both treatments. Perhaps one reason for this finding is because youth in that sample were older and had a broader age range distribution than what is typically found in RCT for youth anxiety and its disorders (i.e., 7 to 16 years old; e.g., Silverman \& Motoca, 2011; Silverman et al., 2008). It is possible that at least a subgroup of youth in each treatment experienced reductions in externalizing problem behaviors, though this remains unknown because possible differences in secondary outcomes as a function of youth age were not pursued. This possibility highlights the importance of examining secondary outcomes as a function of youth characteristics. Examining secondary outcomes as a function of youth characteristics (i.e., sex and age) is a research aim in the present study. 


\section{Reductions in Externalizing Symptoms in GCBT}

Barrett (1998) examined the relative efficacy of GCBT $(n=23)$, GCBT plus parent management (GCBT-PAM; $n=17)$ and a WLC $(n=20)$ along externalizing problem behaviors in a sample of youth (ages 7 to 14 years). Significantly greater pretreatment to posttreatment reductions in externalizing problem behaviors were found in the two GCBT approaches relative to the WLC. There were no significant differences between GCBT and GCBT-PAM on reductions in externalizing problem behaviors. Follow-up data were not reported.

\section{Summary of Studies on Secondary Outcomes}

In summary, the efficacy of anxiety treatments along secondary or non-targeted problems has been demonstrated in six out of seven studies. The only exception was Nauta et al. (2003). The studies reviewed represent initial valuable efforts toward advancing the knowledge base on the efficacy of anxiety treatments along secondary or non-targeted problems. Although extant studies provide initial support for the positive effects of treatment on secondary or non-targeted problems, this research leaves several gaps in knowledge that are described and addressed through the five aims below.

Secondary Outcomes in the Present Study

Treatment Outcome. With rare exceptions (i.e., Silverman et al., 1999a in terms of depressive symptoms; Pina et al., 2003 in terms of externalizing problem behaviors), the effects of anxiety CBTs on secondary or non-targeted problems have been examined with predominately European American participants because they were the most available to researchers. Treatment studies have rarely included ethnic minority groups, particularly Hispanic Latino participants (e.g., Silverman et al., 1999a, Silverman et al., 1999b). The 
low representation of Hispanic Latino participants in treatment studies mirrors, in part, the era of efficacy trials during which they were conducted. Prior to Kendall (1994) there was no evidence from RCTs that anxiety let alone secondary problems could be reduced. RCTs during the 1990s therefore focused on designing treatments and showing that they are efficacious or work to reduce anxiety problems.

That the majority of RCTs were conducted with European American youth also means studies on secondary outcomes drew on these samples. The higher representation of Hispanic Latino youth in Silverman et al. (1999) and subsequent examinations of secondary outcomes (Pina et al., 2003) came about because this team of investigators recruited from the population of Miami-Dade County, Florida, which is largely Hispanic Latino (1.3 million; $57.3 \%$ of the county population; $12.5 \%$ of the population in the Unites States; U.S. Department of Commerce, 2002). Because Pina et al. (2003) drew on Silverman et al.'s RCTs, both of which were conducted during the era of efficacy trials, the specific aim of that study were similar to those of other studies on secondary outcomes: to produce empirical evidence for treatment efficacy.

The first specific aim within the second objective was to evaluate whether anxiety treatment yields reductions in secondary problems in a relatively larger sample of Hispanic Latino youth. Given that past research on secondary outcomes with Hispanic Latino youth, included mostly phobic youth. Only Pina et al. (2003) included youth with anxiety disorders more broadly but did not examine depressive symptoms. It is not yet known whether or not there are favorable secondary outcomes associated with different CBT approaches when applied to youth experiencing anxiety disorders more broadly. The present study will have another look in an independent sample of predominately 
Hispanic Latino youth who were treated for other forms of anxiety with different CBT approaches. Having another look at secondary treatment outcomes in an independent sample will add to the scant literature on secondary treatment outcomes. Evaluation of secondary treatment outcomes in an independent sample of Hispanic Latino youth may advance understanding about the generalizability (or lack thereof) of positive treatment effects from anxiety to related problems in this population when different CBTs are applied to youth experiencing a broad range of anxiety disorders. Knowledge that emerges from this evaluation could have theoretical and practical value for classification efforts and the application of treatment for anxiety and its disorders (e.g., Kraemer et al., 2002).

Treatment Approach and Youth Characteristics. There is limited knowledge on mean level differences in the effects of treatment on secondary or non-targeted problems as a function of approach (i.e., CBT/P versus GCBT) and youth characteristics (i.e., youth sex and age). In terms of treatment approach, although CBT reduces depressive symptoms and externalizing problem behaviors, the comparison approaches in past research are limited (Suveg et al., 2009). No study has compared the relative efficacy of CBT/P and GCBT along depressive symptoms and externalizing problem behaviors. This is because no existing RCTs compared the relative efficacy of CBT/P and GCBT for youth anxiety and its disorders in the same investigation. Studies on secondary outcomes would need to draw on such RCTs. It is therefore not yet known whether CBT/P and GCBT vary in their relative efficacy to reduce depressive symptoms and externalizing problem behaviors. 
Indeed, although reductions in secondary outcomes likely occur in both CBT/P and GCBT, differences as a function of treatment approach are reasonable and may vary by secondary problems. It is possible GCBT relative to $\mathrm{CBT} / \mathrm{P}$ may have a greater therapeutic relevance for reducing depressive symptoms. As described earlier, depressed youth have difficulties with social skills and peer-youth relationships (Hammen et al., 2003; Joiner et al., 1999; Lewinsohn, 1974). A treatment approach that targets difficulties with social skills and peer-youth relationships (i.e., GCBT) therefore may lower depressive symptoms more than a treatment program that does not (i.e., CBT/P).

It is also possible $\mathrm{CBT} / \mathrm{P}$ relative to GCBT may have a greater therapeutic relevance for reducing externalizing problem behaviors. Anxious youth have difficulties regulating not only anxiety, but also externalizing behaviors (Suveg \& Zeman, 2004). As described earlier, CBT/P and GCBT may differ in the extent to which the child's parents or the child himself or herself control the child's behavior. A treatment approach that equips parents with skills to set appropriate contingencies for successful child behavior (i.e., CBT/P) may lower externalizing problem behaviors than a treatment program that relies relatively more on the child's own abilities to regulate emotions (i.e., GCBT). Comorbidity may not only moderate the impact of treatment as hypothesized earlier but treatment may also have an impact on comorbidity and this may vary by treatment approach.

In terms of youth characteristics, with the exception of Kendall et al. (2008), none of the studies reviewed examined whether reductions in secondary outcomes vary as a function of youth sex and age. Such examinations are challenging because most RCTs are characterized by small sample sizes and wide age ranges (e.g., Silverman et al., 2008). 
Examinations of secondary outcomes as a function of youth characteristics are reasonable because CBTs are grounded on a "traditional model of expert-delivered education" and some investigators suggest "boys and older children are more difficult to engage in programs of this type" (p. 366, Lyneham \& Rapee, 2011). The second specific aim within the second objective was to evaluate the efficacy of anxiety treatments in reducing secondary problems as a function of approach (i.e., CBT/P versus GCBT) and youth characteristics (i.e., sex and age).

Mediation of Change. Although several studies reported positive effects of treatment on secondary problems, the mediators responsible for reductions in depressive symptoms and externalizing problem behaviors have not been examined. The present study extends past research because it is the first to examine whether changes in primary anxiety outcomes significantly mediate changes in secondary outcomes. The third specific aim within the second objective therefore was to evaluate whether reductions in anxiety symptoms significantly mediate changes in secondary problems.

Evaluations of mediators are important for theoretical, empirical, and clinical reasons. Theoretically, such examinations may advance understanding about the processes by which treatment produces positive changes in youths' depressive and externalizing problems. This understanding is important given that empirical knowledge about mechanisms and theories of change in child psychotherapy is presently scarce (e.g., Kazdin, 2001). This understanding is also important because it can inform classification efforts with respect to psychopathology. Empirically, evaluation of mediators could set the stage for future investigations into the reasons why anxiety is often comorbid with depressive symptoms and externalizing problem behaviors (e.g., Mathew, Pettit, 
Lewinsohn, Seeley, \& Roberts, 2011). Clinically, evaluation of mediators could yield knowledge that may inform the development of more efficient and cost effective treatments to reduce not only anxiety but also depressive and externalizing problems. Such treatments are presently in high demand (Norton, Hayes, \& Hope, 2004).

Directionality of Change. The temporal course of youth anxiety and comorbid problems has been identified as an important aspect of comorbidity in need of further study (Angold \& Costello, 1993). Of interest is whether youth anxiety increases depressive symptoms and externalizing problem behaviors and whether the reverse is also true. Although bidirectional influences have been incorporated in theoretical models of anxiety and comorbid problems in youth, studies have typically focused on unidirectional influences on the development, maintenance and change of youth anxiety and comorbid problems. Few studies have examined the relation between anxiety and depression over time (e.g., Gallerani, Garber, \& Martin, 2010; Pine et al., 1998). Pine et al. (1998) found major depressive disorders in adolescence significantly predicted a threefold increase in the risk for an anxiety disorder in adulthood.

Intervention designs are suited to clarify issues of directionality of change (Wood, McLeod, Sigman, Hwang, \& Chu, 2003). Only one study in the child anxiety literature examined the issue of directionality of change though an application of parent to child, child to parent and bidirectional influences to the study of youth anxiety and parenting behaviors (Silverman et al., 2009). The results provided preliminary evidence for the bidirectional influences between youth anxiety and parenting behaviors. In that study, the child to parent influence was stronger than the traditional parent to child influence. Given 
that bidirectional influences are theoretically and empirically plausible, it is important to consider these influences in the relation between youth anxiety and comorbid problems.

The fourth specific aim within the second objective therefore was to evaluate the directionality of change in the hypothesized mediated relations between reductions in anxiety symptoms and changes in secondary problems. Evaluation of directionality may point to developmental cascades by which changes in one emotional domain (i.e., anxiety) spread to influence changes in other emotional and behavioral domains (i.e., depressive symptoms and externalizing problem behaviors) (e.g., Masten et al., 2005). Some investigators, for example, believe successful development is the result of the acquisition of social, emotional, and cognitive competences (e.g., Cicchetti \& SchneiderRosen, 1986). Failure to acquire such competences is theorized to influence the development of internalizing and externalizing problems by constraining the individuals' abilities for dealing with daily challenges and increasing vulnerability to stress (Masten, 2006; Patterson \& Capaldi, 1990; Sameroff, 2000).

In dynamic systems theory, more specifically, changes in one area of emotional competency are said to set in motion a cascade of consequences that ultimately have large developmental effects (Sameroff, 2000; Thelen, 1989). According to Thelen (1989), "changes in any one domain therefore may become amplified and have system-wide reverberations" (p. 94). Inherent in all theories of development that focus on the transactions among individuals is the possibility of spillover effects that result from dynamic interactions over time. Anxiety and depressive symptoms, on the one hand, and anxiety symptoms and externalizing problem behaviors, on the other hand, may be linked through a developmental cascade from one type of difficulty to the other. Treatments that 
arrest such progressions of maladaptive developmental pathways could provide an understanding, even if indirect, of positive and negative cascades in development (e.g., Masten et al., 2005; Van Lier \& Koot, 2010).

Indeed, when it comes to the directionality of change, four scenarios are feasible: (1) changes in primary anxiety symptoms are associated with changes in secondary problems but not vice versa, (2) changes in secondary problems are associated with changes in primary anxiety symptoms but not vice versa, (3) changes in both primary anxiety symptoms and secondary problems are associated with changes in each other, and (4) changes in primary anxiety symptoms are not associated with changes in secondary problems. Examining whether changes flow not only from primary anxiety symptoms to secondary problems but also from secondary problems to anxiety symptoms will begin to illuminate the likely complex nature of the proposed mediated relations between primary anxiety and secondary outcome problems. Examining the directionality of change between anxiety and comorbid problems is also an important initial step toward advancing evidence based-explanations of treatment (Silverman \& Motoca, 2011).

Moderated Mediation. So long as questions of mediation and directionality of change have not been examined, evaluations of possible differences in the relations between primary anxiety and secondary outcomes as a function of treatment approach (i.e., $\mathrm{CBT} / \mathrm{P}$ versus $\mathrm{GCBT}$ ) and youth characteristics (i.e., sex and age) are also lacking (i.e., moderated mediation). The fifth specific aim within the second objective was to evaluate whether the hypothesized mediated relations between changes in primary anxiety symptoms and secondary outcome problems are moderated by treatment approach (CBT/P versus GCBT) and youth characteristics (sex and age). Evaluation of 
moderated mediation would provide a closer look into the likely complex relations between changes in primary anxiety and secondary outcome problems.

If significant moderators emerge this would suggest there are differences in the function, direction or strength of the hypothesized mediated relations for youth in CBT/P versus GCBT, boys versus girls, and younger versus older children. Understanding whether these differences exist would inform for whom and under what conditions reductions in primary anxiety symptoms result in changes in secondary outcome problems. Clarifying the complex temporal relations between primary anxiety and secondary outcomes as a function of youth characteristics could inform future investigations about the processes underlying these treatments and guide the adaptation of sex and age specific approaches (e.g., Gallerani et al., 2010).

Specificity of Effects. Some investigators suggest that at least two types of anxiety can be found in youth (i.e., separation anxiety, fears, obsessions and compulsions versus general anxiety and perhaps social anxiety) (e.g., Lahey et al., 2004). General and social anxiety symptoms could also be part of the same dimension as depressive symptoms (e.g., Lahey et al., 2004). Some exploratory factor analyses of youth self-ratings, for example, have yielded a single dimension of anxiety and depression (Achenbach, 1991b). Thus, when examining the relations between changes in anxiety and secondary depressive symptoms (i.e., third through fifth aim within the second objective above), the present study will distinguish between two types of anxiety symptoms (i.e., general and social anxiety). The distinction between general and social anxiety in the present study when examined in relation to depression may inform classification efforts, such as 
whether general and social anxiety symptoms are distinct from each other and from depressive symptoms.

\section{The Present Study}

Evident from the Literature Review, the two main issues of interest in the present study - treatment moderation and secondary outcomes - have been examined from the vantage points of two related, yet disparate lines of psychotherapy research. The present study will integrate these two lines of psychotherapy research into one investigation of treatment moderation and secondary outcomes. This integration is achieved through examining comorbidity, a modifiable youth characteristic, as a treatment moderator, as well as a secondary outcome. In addition, youth age, a non-modifiable youth characteristic, will be examined as a moderator of treatment outcome. This integration is achieved via an analysis of data from an existing RCT of CBT/P and GCBT for youth anxiety and its disorders. The following research questions and related hypotheses mapping onto the study's two objectives will be examined.

Objective I. The first objective of the present study was to tests for youth age, comorbidity with depressive disorders and comorbidity with externalizing disorders as moderators of treatment outcome at posttreatment and 12 month follow up.

Developmental psychopathology theory and research have guided the selection of these variables as potential moderators of treatment outcome.

Research Question 1.1. Is youth age a moderator of treatment outcome?

Hypothesis 1.1. Youth age will be a moderator of treatment outcome. CBT/P relative to GCBT may lower anxiety symptoms more for younger than older children. Put 
differently, GCBT relative to CBT/P may lower anxiety symptoms more for older than younger children.

Research Question 1.2. Is comorbidity with depressive disorders a moderator of treatment outcome?

Hypothesis 1.2. Comorbidity with depressive disorders will be a moderator of treatment outcome. Two competing hypotheses are feasible to describe the direction of the interaction.

Hypothesis 1.2.1. GCBT relative to CBT/P may lower anxiety symptoms more for youth with comorbid depressive disorders than youth without comorbid depressive problems. Put differently, CBT/P relative to GCBT may lower anxiety symptoms less for youth with comorbid depressive disorders than youth without comorbid depressive disorders.

Hypothesis 1.2.2. CBT/P relative to GCBT may lower anxiety symptoms more for youth with comorbid depressive disorders than youth without comorbid depressive disorders.

Research Question 1.3. Is comorbidity with externalizing disorders a moderator of treatment outcome?

Hypothesis 1.3. Comorbidity with externalizing disorders will be a moderator of treatment outcome? GCBT relative to CBT/P may lower anxiety symptoms less for youth with externalizing problem behaviors than youth without comorbid externalizing problem behaviors.

Simple Main Effects. The present study also pursued exploratory simple main effects to evaluate: (1) the differential outcome of the treatment approaches as a function 
of youth characteristics; and (2) the relative efficacy of the treatment approaches at each level of the moderators.

Objective II. The second objective of the present study was to evaluate the efficacy of psychosocial treatments aimed at reducing anxiety and its disorders along secondary outcomes (depressive symptoms and externalizing problem behaviors) at posttreatment and 12-month follow up.

Research Question 2.1. Are there statistically significant positive changes in secondary, non-targeted, problems following a treatment designed for anxiety and its disorders?

Hypothesis 2.1. There will be significant positive changes in secondary, nontargeted, problems following a treatment designed for anxiety and its disorders.

Research Question 2.2. Are the hypothesized positive changes in secondary, nontargeted, problems following treatment statistically significantly different in CBT/P relative to GCBT?

Hypothesis 2.2. In the absence of past research comparing the relative efficacy of CBT/P and GCBT along secondary, nontargeted, problems no hypothesis is made for this research question.

Research Question 2.3. Are the hypothesized positive changes in secondary, nontargeted, problems following treatment statistically significantly different as a function of youth sex and age?

Hypothesis 2.3. Because there is limited knowledge on mean-level differences in the effects of treatment on secondary, nontargeted, problems as a function of youth sex and age, no hypothesis is made for this research question. 
Research Question 2.4. Are reductions in youth anxiety symptoms significant mediators of change in secondary, non-targeted, problems (i.e., youth depressive symptoms, measured by youth rated CDI; youth externalizing problem behaviors, measured by parent rated CBCL-E)?

Hypothesis 2.4. Reductions in youth anxiety symptoms will be significant mediators of changes in secondary, non-targeted, problems (i.e., youth depressive symptoms, measured by youth rated CDI; youth externalizing problem behaviors, measured by parent rated CBCL-E)?

Research Question 2.5. What is the directionality of change in the relation between reductions in youth anxiety symptoms and changes in youth secondary, nontargeted, problems?

Hypothesis 2.5. The directionality of change in the relation between reductions in youth anxiety symptoms and changes in youth secondary, non-targeted, problems will be reciprocal. As anxiety symptoms decrease, secondary, non-targeted, problems will also decrease and vice versa.

Research Question 2.6. Are the hypothesized bidirectional relations between reductions in youth anxiety symptoms and changes in youth secondary, non-targeted, problems moderated by treatment approach (i.e., CBT/P; GCBT) and youth characteristics (i.e., sex and age)?

Hypothesis 2.6. Due to the lack of research with regard to the moderating effects of treatment approach and youth characteristics on the hypothesized mediated relations between reductions in youth anxiety symptoms and changes in youth secondary, nontargeted, problems, no hypothesis is made for this research question. 
In evaluating the efficacy of treatment along secondary outcomes, the present study used youth report for depressive symptoms and parent report for externalizing problem behaviors in separate analyses. This is because studies suggest that particular informants may be optimal for different types of problems (Lahey et al., 2004). Youth have consistently been shown to be useful informants for anxiety and depressive symptoms but not externalizing problem behaviors (e.g., Bird, Gould, \& Staghezza, 1992). According to Lahey et al. (2004), youth may be unable to contribute valid information on externalizing behaviors because these problems require judgments about broad aspects of behavior relative to other youth. Youth are able, however, to contribute valid information on anxiety and depressive because these symptoms characterize more specific emotions. 


\section{CHAPTER III}

\section{METHODOLOGY}

\section{Participants}

Participants in the present study were 183 youth who completed treatment (ages 6 to 16 years; $M=9.72$ years; $S D=2.21 ; 54 \%$ males) and their mothers who presented to the Child Anxiety and Phobia Program (CAPP) at Florida International University (FIU). The youth's age range of 6 to 16 years represents the modal age range of the age of onset of separation anxiety disorder (SAD), social phobia (SOP), specific phobia, (SP), and generalized anxiety disorders (GAD) in the population and is representative of CAPP's referral patterns. Participants were referred to CAPP by pediatricians, school counselors, and other mental health professionals. About 75 percent $(n=137)$ of the sample were Hispanic Latinos, 19.7 percent $(n=36)$ were European Americans, 2.7 percent $(n=5)$ were African Americans, and $2.7(n=5)$ were of other ethnic backgrounds.

The study's inclusion criteria included the following, all youth: (A) met criteria for a primary diagnosis for a DSM IV anxiety disorder of SAD, SOP, SP, and GAD. All diagnoses were reviewed and confirmed at a staff conference directed by Dr. Silverman following the administration of the ADIS C/P (Silverman \& Albano, 1996), (B) received a mean score of 4 or greater on the Clinician's Rating Scale of Severity (see Measures), (C) ceased all other psychosocial treatment upon review with the Center's clinic staff and the service provider, and (D) withdrew from certain psychopharmacological agents viewed as confounding the study, upon review with the Center's psychiatric consultant, (E) were between 6 and 16 years old, and $(\mathrm{F})$ had parents or guardians who agreed to participate in the youth's treatment. 
The study's exclusion criteria included the following, youth who (A) met as a primary diagnosis any Axis 1 DSM-IV disorder other than SAD, SOP, SP, and GAD; or (B) failed to withdraw from psychosocial treatment or psychopharmacological agents as per study protocol and as per medical supervision; or (C) youth and/or parents met diagnoses for any one of the following -- Pervasive Developmental Disorders, Mental Retardation, Organic Mental Disorders, Schizophrenia and Other Psychotic Disorders; or (D) youth and/or parents showed high likelihood and/or serious intent of hurting themselves or others.

Measures

Anxiety Disorders Interview Schedule for DSM-IV: Child and Parent Versions (ADIS for DSM-IV: C/P; Silverman \& Albano, 1996). The ADIS for DSM-IV: C/P was administered to the youth and mother to assess anxiety and related disorders. Interviewers assigned diagnoses that youth and mother agreed were most interfering. In cases of disagreement, the interviewer considered both informants' views to derive a final diagnosis. In cases of multiple diagnoses, the relative interference of each disorder was determined by obtaining interference ratings from each source and prioritizing each disorder from most to least interfering or disturbing. The disorder deemed most interfering/disturbing was viewed as primary and was targeted in treatment. In addition to a primary anxiety diagnosis serving as a study inclusion criterion, diagnostic status was an index of clinically significant improvement. The ADIS for DSM-IV: C/P has satisfactory to good reliability for specific diagnoses and symptom patterns as well as strong correspondence with youths' anxiety self-ratings (Silverman, Saavedra, \& Pina, 2001). 
Children's Manifest Anxiety Scale - Revised (RCMAS; Reynolds \& Richmond, 1978). Child anxiety treatment response was assessed on a specific symptom level from the child's perspective using the RCMAS. The RCMAS is a 37-item self-rating scale to which children respond either yes (scored 1) or no (scored 0 ) to anxious symptoms. Pela and Reynolds (1982) reported excellent test-retest reliability $(r=.98)$ using a three-week interval. Significant correlations have been found between the Total Anxiety scale, trait anxiety, and fear ( $r \mathrm{~s}=.63$ to .88$)$ (Ollendick, 1983). The RCMAS has been used as the primary outcome measure in almost all past childhood anxiety trials and has been found to be a sensitive measure of change (e.g., Silverman et al., 1999a, b). The alpha coefficient in the present sample was .63.

Multidimensional Anxiety Scale for Children (MASC; March, Parker, Sullivan, Stallings, \& Connors, 1997). Child anxiety treatment response was assessed on a specific symptom level from the child's perspective also using the MASC. The MASC is increasingly being used in childhood anxiety research because it provides for a more comprehensive assessment of childhood anxiety. The MASC consists of 39 items distributed across four factors: Physical Symptoms, Social Anxiety, Harm Avoidance, and Separation Anxiety. Children's ratings of anxiety symptoms are made on the MASC via a 4-point Likert scale. The MASC's test-retest reliability using a 3-week and 3-month interval is satisfactory to excellent (ICCs of .93 and .87 , respectively). Adequate convergent and divergent validity also has been demonstrated in that shared variance was highest for scales sampling symptom domains of anxiety, intermediate for depression, and lowest for externalizing symptoms (March et al., 1997). Only the MASC Social 
subscale was used in the present study. The alpha coefficient for MASC Social in the present sample was .83 .

Revised Children's Manifest Anxiety Scale (Parent Version; RCMAS/P). The wording of RCMAS items was changed from, "I..." to "My child..." as done in past research (e.g., Kendall, 1994; Silverman et al., 1999). Twenty-eight items are rated either Yes or No and scored 1 or 0 . Summed items yield a Total Anxiety score. The alpha coefficient for RCMAS Total in the present sample was .69.

Children's Depression Inventory (CDI; Kovacs, 1981). The CDI contains 27 items designed to assess cognitive, affective, and behavioral symptoms of youth depression. The CDI has a test-retest reliability of .72 (Smucker, Craighead, Craighead, \& Green, 1986). The CDI has been found to discriminate between psychiatric and nonclinic samples; the CDI also has been found to correlate with clinicians' independent global depression ratings ( $r=.55$; Kovacs, 1981). The alpha coefficient for CDI Total in the present sample was .86 .

Child Behavior Checklist (CBCL; Achenbach, 1991) is a 113-item measure that assesses children's behavior problems. Each item is scored on a 3-point scale $(0=n o t$ true, 1 = sometimes true, 2 = often true). The CBCL provides scores for the total scale, as well as for the Internalizing and Externalizing subscale. Parents' ratings on the CBCL's Externalizing subscale (CBCL-E) were used as indicator of secondary treatment outcome in the present study. CBCL scaled scores have been found to discriminate between clinicreferred and non-referred youth (Achenbach, 1991). 


\section{Procedures}

Assessment interviews and questionnaires were administered after parents provided informed consent and youth provided informed assent. Assessment interviews and questionnaires were generally completed in one session by one diagnostician (a doctoral level student). During the child interview, the parent was administered a questionnaire battery. Following the child interview, the parent was interviewed and the child was administered a questionnaire battery. Measures were administered to all youth and parents at pretreatment, at posttreatment and at 12 month follow-up.

\section{Treatment Approaches and Therapists}

Participants were randomly assigned to $\mathrm{CBT} / \mathrm{P}$ or GCBT in blocks of seven. The specific approach (CBT/P or GCBT) used to start the random assignment process was determined by the toss of a coin. Assignment to treatment in blocks of seven was used to avoid delay in the approachion of groups. Treatment manuals for CBT/P and GCBT were developed to standardize the content of each treatment session. Nevertheless, therapists were advised to consider the developmental needs of the youth and proceed accordingly with the treatment protocol. Given the high proportion of Hispanic families in the sample, $8 \%$ of the treatments were delivered in a bilingual approach (English and Spanish) by the request of the parent participating in $\mathrm{CBT} / \mathrm{P}$. All group treatments were delivered in English. An outline of the basic core program as presented to participants was presented in detail by Marin (2010). A brief review of the program is provided below.

In $\mathrm{CBT} / \mathrm{P}$, the youth and parents met with the therapist for a total of 60 minutes. CBT/P involved targeting of parenting skills and parent-youth relationships. The main therapeutic components involved training parents in managing their children's anxiety 
and avoidant behaviors, having parents set appropriate contingencies for successful child non-avoidance, and helping parents to enhance their acceptance/warmth toward their children with improved communication and problem-solving skills.

In GCBT, the youth met in the group with the therapist for a total of 60 minutes. GCBT involved targeting of social skills and peer-youth relationships. The main therapeutic components involved training children in being more helpful and positive toward other children in the group in the context of anxiety reduction, having children accept the help and support of other group members in role-plays, and training children in social skills (e.g., compliment giving, conversational skills). The parents of the youth who were assigned to GCBT also had three brief group meetings (about 30 minutes) with each group therapist (at the start of the treatment program, the middle, and the end) to be kept abreast about the program and the youth's tasks and activities similar to past RCT (e.g., Barrett, 1998; Flannery-Schroeder \& Kendall, 2001). Parents were not actively incorporated or involved in the youth's treatment in GCBT, thereby ensuring GCBT's distinctiveness from CBT/P in terms of their targeting distinct contextual variables. The total number of sessions in both $\mathrm{CBT} / \mathrm{P}$ and GCBT was 12 to 14 sessions. Because the two treatment approaches required similar therapeutic skill levels therapists were crossed between treatments as recommended by Kazdin (1994). All therapists received training in the proper administration of the treatments by Dr. Silverman. Emphasis was placed on highlighting the overlap between the treatment approaches (e.g., youth exposure) but also in ensuring therapists understood the distinctions between the two. Dr. Silverman conducted weekly supervision meetings with therapists to prepare for upcoming sessions and process sessions just completed. This 
included the review of the therapists' treatment notes, listening to a random selection of therapists' session tapes and providing ongoing feedback via instructions and role plays. Eight doctoral level graduate students in psychology delivered the treatments. There were no statistically significant differences between the therapists on any of the primary outcome variables (Marin, 2010). 


\section{CHAPTER IV}

\section{RESULTS}

\section{Preliminary Analyses}

Outliers. Outlier analyses were undertaken prior to all major analyses. The analyses were both nonmodel based and model based. For the former, multivariate outliers were identified by examining leverage indices for each individual and defining an outlier as a leverage score four times greater than the mean leverage. There were no outliers found in the data using this approach. An additional approach was to use model based outlier analysis. This involved randomly selecting an indicator for each variable and then regressing that indicator onto an indicator for variables that the endogenous variable is assumed to be a linear function of. This analysis uses ordinary least squares regression in a limited inapproachion estimation framework. Standardized dfbetas were examined for each individual and for each predictor, as well as the intercept. An outlier was defined as any case with an absolute standardized dfbeta larger than 1.0. There were no outliers found in the data using this approach.

Non-Normality. Univariate indices of skewness and kurtosis were examined to determine whether the absolute value of any of these indices was greater than 2.0. Nonnormality was evident in several of the variables. To account for the non-normality, all analyses were pursued in MPlus 6 (Muthén \& Muthén, 2007) by using a maximum likelihood estimator robust to violations of normality based on the Huber-White algorithm (Huber, 1967; White, 1980). 
Analysis of Missing Data. The first step in the analysis of missing data was to determine if there was systematic bias in the patterning of missing data. For a given measure, a dummy variable was constructed to indicate the presence or absence of missing data on that measure. Associations between these dummy variables and demographic as well as other study variables were examined. No significant associations were observed, indicating that there was no systematic bias in the patterning of missing data.

\section{Objective I: Treatment Moderation}

1.1. Interaction Contrasts. Multiple regression analyses were used to examine youth sex, comorbidity with depressive disorders and comorbidity with externalizing disorders as moderators of treatment outcome. The treatment approaches and the categorical moderators were dummy coded. Continuous moderators were mean centered to allow for easy interpretation of the regression coefficients and circumvent the potential for multicollinearity (Aiken \& West, 2003; Jaccard \& Turrisi, 2003). Product terms were created between the dummy coded treatment variable and each of the moderators. A multiple regression model was run for each of the moderators using pretreatment anxiety, the dummy-coded treatment, the moderator, and the product term to predict posttreatment and follow-up anxiety scores on the RCMAS and RCMAS/P, respectively. The statistical significance of the product term in the multiple regression models was used to declare moderation. To test if the efficacy of CBT/P relative to GCBT was statistically significantly different at one level of the moderator compared to another, single degree of freedom interaction contrasts were pursued. This corresponds to the last row within each set of comparisons in Table 1. For example, the single degree of freedom interaction 
contrast for youth age compared the mean difference for younger versus older children in $\mathrm{CBT} / \mathrm{P}$ with the mean difference for younger versus older children in GCBT.

\subsection{Simple Main Effects. To examine whether CBT/P and GCBT yielded}

differential outcomes at each level of the moderator variables, simple main effects were pursued. This corresponds to the first two rows within each set of comparisons in Table 1. To test whether the proposed moderator variables yield differential outcomes within each treatment approach, an additional set of simple main effects were pursued. This corresponds to the third and fourth rows within each set of comparisons in Table 1. Given that a limited inapproachion estimation approach was used, no model fit indices are reported. Each moderator will be discussed in turn below.

Youth Age. Age did not moderate treatment outcome based on youth report both at posttreatment $(B=0.42, S E=0.42,95 \% \mathrm{CI}-0.40$ to $1.24, p>.05)$ and at 12 month follow-up $(B=0.25, S E=0.43,95 \% \mathrm{CI}=-0.59$ to $1.09, p>.05)$. The simple main effects examining the outcome of each treatment approach as a function of age were not statistically significant; nor were the simple main effects examining the relative efficacy of CBT/P and GCBT separately for younger and older children (see Table 1).

Age moderated treatment outcome based on parent report at posttreatment $(S E=$ $.87, S E=.31,95 \% \mathrm{CI}=0.34$ to $1.37, p=.01)$ but not at 12 month follow-up $(B=.52, S E$ $=0.34,95 \% \mathrm{CI}=-0.15$ to 1.18$)$. The relative difference in the mean residual anxiety scores between $\mathrm{CBT} / \mathrm{P}$ and GCBT at posttreatment was $-3.67, p<.05$ at age $6 ;-2.80$ at age $7, p<.05 ;-1.93$ at age $8, p>.05 ; 2.40,-1.07$ at age $9, p>.05 ;-.20$ at age $10, p>.05$; .67 at age $11, p>.05 ; 1.53$ at age $12, p>.05 ; 2.4$ at age $13, p<.05 ; 3.27$ at age $14, p<$ $.05 ; 4.13$ at age $15, p<.05 ; 5.01$ at age $16, p<.05$. For every one year increase in age 
GCBT lowered anxiety scores by .87 units more than CBT/P, adjusting for pretreatment anxiety scores. For children 6 through 8,CBT/P yielded a statistically significantly lower mean residual anxiety score than GCBT. For children 9 through 12, CBT/P and GCBT did not differ in their mean residual anxiety scores. For children 13 through 16, GCBT yielded a statistically significantly lower mean residual anxiety score than $\mathrm{CBT} / \mathrm{P}$. CBT/P relative to GCBT therefore lowered anxiety scores more for younger children than older children. GCBT relative to CBT/P lowered anxiety scores more for older children than younger children.

Guided by the above analyses, the sample was divided into younger (6 to 11 years) and older children (12 to 16 years) in order to examine simple main effects and graph the interaction. The multiple regression model was re-ran using the dummy-coded treatment, dummy coded age (younger children $=0$; older children $=1$ ) and their product term predicting posttreatment scores on the RCMAS/P. The results of this analysis were consistent with the above findings with one exception discussed below. The path coefficient for the product term predicting posttreatment scores on the RCMAS/P was statistically significant $(B=5.18, S E=1.75,95 \% \mathrm{CI}=0.89$ to $6.46, p<.01)$.

The mean residual posttreatment anxiety for older youth in CBT/P was 8.80; the mean residual posttreatment anxiety for older youth in GCBT was 4.89; their mean difference was $3.91(p=.01)$. GCBT lowered anxiety symptoms more for older youth than CBT/P. The mean residual posttreatment anxiety for younger youth in CBT/P was 8.01; the mean residual posttreatment anxiety for younger youth in GCBT was 9.26; their mean difference was $-1.26(p>.05)$. Using the dummy coded approach to examining youth age $\mathrm{CBT} / \mathrm{P}$ and GCBT did not differ on anxiety symptom reduction when it came 
to younger children. The mean residual posttreatment anxiety for older youth in CBT/P was 8.80; the mean residual posttreatment anxiety for younger youth in $\mathrm{CBT} / \mathrm{P}$ was 8.01 ; their mean difference was $.80, p>.05$. The mean residual posttreatment anxiety for older youth in GCBT was 4.89; the mean residual posttreatment anxiety for younger youth in GCBT was 9.26; their mean difference was $-4.37, p<.05$. GCBT lowered anxiety symptoms more for older youth than younger youth. The interaction was $5.18, p<.01$. GCBT relative to CBT/P lowered anxiety scores more for older children than younger children (see Figure 12).

Comorbidity with Depressive Disorders. Comorbidity with depressive disorders did not moderate treatment outcome based on youth report both at posttreatment $(B=$ $0.20, S E=3.59,95 \% \mathrm{CI}=-6.85$ to $7.24, p>0.05)$ and 12 month follow-up $(B=-0.54, S E$ $=2.82,95 \% \mathrm{CI}=-6.06$ to $4.98, p>.05)$. Using youth report, the simple main effects examining the outcome of each treatment as a function of comorbidity with depressive disorders were not statistically significant both at posttreatment and 12 month follow-up. The simple main effects examining the relative efficacy of CBT/P and GCBT separately for youth with and without comorbid depressive disorders were also not statistically significant both at posttreatment and 12 month follow-up (see Table 1).

Comorbidity with depressive disorders moderated treatment outcome based on parent report at posttreatment but not at 12 month follow-up. The path coefficient for the product term between dummy-coded treatment and dummy-coded comorbid depressive disorders was statistically significant $(B=6.28, S E=2.51,95 \% \mathrm{CI}=0.67$ to $10.41, p=$ $.01)$. The mean residual posttreatment anxiety for youth with comorbid depressive disorders in $\mathrm{CBT} / \mathrm{P}$ was 10.12 ; the mean residual posttreatment anxiety for youth with 
comorbid depressive disorders in GCBT was 4.67; their mean difference was 5.45, $p=$ .02. GCBT relative to CBT/P lowered anxiety scores by 5.45 units more for youth with comorbid depressive disorders, adjusting for pretreatment anxiety scores. Put differently, $\mathrm{CBT} / \mathrm{P}$ relative to GCBT lowered anxiety scores by 5.45 units less for youth with comorbid depressive disorders. The mean residual posttreatment anxiety for youth without comorbid depressive disorders in $\mathrm{CBT} / \mathrm{P}$ was 8.01 ; the mean residual posttreatment anxiety for youth without comorbid depressive disorders in GCBT was 8.84; their mean difference was $-.80, p>.05$. CBT/P relative to GCBT did not differ in their mean residual posttreatment anxiety scores for youth without comorbid depressive disorders. The interaction was $6.28, p=.01$. GCBT relative to $\mathrm{CBT} / \mathrm{P}$ lowered anxiety scores by 6.28 units more for youth with comorbid depressive disorders than youth without comorbid depressive disorders, adjusting for pretreatment anxiety scores (see Figure 13).

Also using parent report, the mean residual posttreatment anxiety for youth with comorbid depressive disorders in $\mathrm{CBT} / \mathrm{P}$ was 10.12 ; the mean residual posttreatment anxiety for youth without comorbid depressive disorders in CBT/P was 8.84; their mean difference was $2.11(p>.05)$ (see Table 1$)$. The mean residual posttreatment anxiety for youth with comorbid depressive disorders in GCBT was 4.67; the mean residual posttreatment anxiety for youth without comorbid depressive disorders in GCBT was 8.84; their mean difference was $-4.17(p=.02)$ (see Table 1$)$. GCBT lowered anxiety scores by 4.17 units more for youth with comorbid depressive disorders than youth without comorbid depressive disorders, adjusting for pretreatment anxiety scores. 
Comorbidity with Externalizing Disorders. Comorbidity with externalizing disorders did not moderate treatment outcome based on youth report at both posttreatment $(B=-1.30, S E=1.95,95 \% \mathrm{CI}=-5.13$ to $2.52, p>.05)$ and 12 month follow-up $(B=0.58, S E=2.41,95 \% \mathrm{CI}=-4.14$ to $5.30, p>.05)$. Using youth report, the simple main effects examining the outcome of each treatment as a function of comorbidity with externalizing problem behaviors were not statistically significant both at posttreatment and 12 month follow-up. The simple main effects examining the relative efficacy of CBT/P and GCBT separately for youth with and without comorbid externalizing disorders were also not statistically significant both at posttreatment and 12 month follow-up (see Table 1).

Comorbidity with externalizing disorders moderated treatment outcome based on parent report at posttreatment but not at 12 month follow-up. The path coefficient for the product term between dummy-coded treatment and dummy-coded comorbid externalizing disorders was statistically significant $(B=-3.88, S E=1.76,95 \% \mathrm{CI}=-7.66$ to $-0.11, p=.04)$. The mean residual posttreatment anxiety for youth with comorbid externalizing disorders in $\mathrm{CBT} / \mathrm{P}$ was 7.87 ; the mean residual posttreatment anxiety for youth with comorbid externalizing disorders in GCBT was 10.82; their mean difference was $-2.94, p=.06$. The mean residual posttreatment anxiety for youth without externalizing disorders in $\mathrm{CBT} / \mathrm{P}$ was 8.26 ; the mean residual posttreatment anxiety for youth without comorbid externalizing disorders in GCBT was 7.35; their mean difference was $.91, p>.05$. The interaction was $-3.85, p=.04$. GCBT relative to CBT/P lowered anxiety scores by 3.85 units less for youth with comorbid externalizing disorders than 
youth without comorbid externalizing disorders, adjusting for pretreatment anxiety scores (see Figure 14).

Also using parent report, the mean residual posttreatment anxiety for youth with comorbid externalizing disorders in $\mathrm{CBT} / \mathrm{P}$ was 7.87 ; the mean residual posttreatment anxiety for youth without comorbid externalizing disorders in CBT/P was 8.26 ; their mean difference was $-0.39(p>.05)$ (see Table 1$)$. The mean residual posttreatment anxiety for youth with comorbid externalizing disorders in GCBT was 10.82; the mean residual posttreatment anxiety for youth without comorbid externalizing disorders in GCBT was 7.35; their mean difference was $3.46(p=.01)$ (see Table 1). GCBT lowered anxiety scores by 3.46 units less for youth with comorbid externalizing disorders than youth without comorbid externalizing disorders, adjusting for pretreatment anxiety scores.

\section{Objective II: Secondary Outcomes: Depressive Symptoms}

2.1.1.Treatment Outcome. To examine whether 12 to 14 weeks of anxiety treatment yields changes in depressive symptoms analyses of variance were pursued in a structural equation modeling (SEM) framework collapsing the outcome across treatments. The SEM equivalent of a $2 X 3$ between-within subjects analysis of variance was conducted on the CDI, with the type of treatment approach (CBT/P and GCBT) representing a between-subjects factor and time (pre and post; post and follow-up) representing a within-subjects factor. In terms of changes in depressive symptoms from pre to post, the main effect for time on the CDI was statistically significant $(B=25.08, p$ $<.001)$. 
Table 4 presents relevant single degree of freedom contrasts and their associated statistics for CDI. The contrasts used non-pooled error terms for the contrasts involving repeated measures but pooled terms for the contrasts across the between-subjects factor. The mean difference for the main effect of time from pre to post collapsing across treatments was 4.31 with post showing a decrease in youth self-ratings of depressive symptoms, on average, relative to pre. In terms of post to 12 month follow-up, the main effect for time on the CDI was statistically significant $(B=24.99, p<.001)$. Table 4 presents relevant single degree of freedom contrasts and their associated statistics for CDI. The contrasts used non-pooled error terms for the contrasts involving repeated measures but pooled terms for the contrasts across the between-subjects factor. The mean difference for the main effect of time collapsing across treatment approach was 0.58 with 12 month follow-up showing a decrease in youth self-ratings of depressive symptoms, on average, relative to post.

\subsubsection{The Relative Efficacy of $C B T / P$ vs. GCBT. Of interest was whether the} positive changes in secondary depressive symptoms following treatment were statistically significantly different in $\mathrm{CBT} / \mathrm{P}$ relative to GCBT at posttreatment and at 12 month follow-up. The interaction effect of treatment approach (CBT/P and GCBT) by time (pre and post) was not statistically significant $(B=-0.73, p>.05)$. The interaction effect of treatment approach (CBT/P and GCBT) by time (post and follow-up) was not statistically significant $(B=-0.28, p>.05)$.

To determine if the pretreatment to postttreatment time difference in depressive symptoms occurred at each treatment approach, simple main effects (SME) contrasts were performed. The difference was statistically significant for both treatments with post 
scores showing a decrease in youth depressive symptoms. The time difference for CBT/P was 3.86 and for GCBT it was 4.76. The significant pretreatment to posttreatment time difference effects were maintained when the Holm modified Bonferroni method was applied to control the experiment-wise error rate at 0.05 .

To determine if the postttreatment to 12 month follow-up time difference in depressive symptoms difference occurred at each treatment approach, simple main effects (SME) contrasts were performed. The time difference was statistically significant for both treatments, with 12 month follow-up scores showing a decrease in youth depressive symptoms relative to posttreatment. The time difference for CBT/P was .42 and for GCBT it was .70. The significant time difference effects were maintained when the Holm modified Bonferroni method was applied to control the experiment-wise error rate at 0.05 .

\subsubsection{Change as a Function of Youth Characteristics. Of interest was whether the} positive changes in secondary depressive symptoms following treatment were statistically significantly different for girls versus boys and younger versus older children. The interaction effect of youth sex and time (pre and post) was not statistically significant ( $z=$ $.23, p>.05$ ). The interaction effect of youth sex and time (post and follow-up) was not statistically significant $(\mathrm{z}=-.23, p>.05)$. The interaction effect of youth age and time (pre and post) was not statistically significant $(z=.27, p>.05)$. The interaction effect of youth age and time was (post and follow-up) not statistically significant $(\mathrm{z}=.05, \mathrm{p}>.05)$.

To determine if the pretreatment to postttreatment and posttreatment to 12 month follow-up time difference in depressive symptoms occurred at each level of the moderator variables, simple main effects (SME) contrasts were performed. The 
difference was statistically significant for boys and girls and younger (6 to 11 years) and older children (12 to 16 years). Posttreatment scores showed a decrease in youth depressive symptoms relative to pretreatment. Twelve month follow-up scores showed a decrease in youth depressive symptoms relative to posttreatment. All statistically significant time difference effects remained significant when the Holm modified Bonferroni method was applied to control the experiment-wise error rate at 0.05 .

SEM. The data were further analyzed using SEM to yield insight into the underlying dynamics depicted in Figure 1. Of interest was whether change in youth anxiety symptoms significantly mediates change in secondary, non-targeted, problems. The SEM analyses require clarification and we use depressive symptoms to explicate the modeling approach (see Figure 1). A two valued dummy variable for the two treatment approaches (CBT/P versus GCBT) was defined and was assumed to outcome youth anxiety and depressive symptoms at the posttreatment (paths $a$ and $b$ in Figure 1). The dummy variable was included in the model to be consistent with the study's design; however, the SEM analyses reported here were first conducted collapsing across the two treatments. Paths $c$ and $d$ represent traditional autoregressive effects. Paths $c$ represents change in anxiety symptoms from pretreatment to posttreatment as an effect of the approach collapsed across treatments. Paths $d$ represents change in anxiety symptoms from posttreatment to 12 month follow-up as an effect of the approach also collapsed across treatments. Paths $e$ and $f$ represent traditional autoregressive effects. Paths $e$ represents change in secondary outcome measures from pretreatment to posttreatment as an effect of the approach collapsed across treatments. Paths $f$ represents change in 
secondary outcome measures from posttreatment to 12 month follow-up as an effect of the approach also collapsed across treatments (see Figure 1).

Paths $g$ and $h$ represent contemporaneous effects and estimate the extent to which changes in anxiety symptoms from pretreatment to posttreatment and posttreatment to 12 month follow-up mediate changes in the secondary outcome measures over the same time period (paths $g$ and $h$, respectively in Figure 1). Path $g$ and $h$ represent contemporaneous reciprocal causality, in that (a) changes in youth anxiety are assumed to outcome changes in depressive symptoms, and (b) changes in depressive symptoms are assumed to outcome changes in youth anxiety.

Paths $i$ and $j$ represent lagged effects. Path $i$ estimates the extent to which changes in secondary outcome measures from pretreatment to posttreatment are associated with changes in primary anxiety symptoms from posttreatment to 12 month follow-up. Path $j$ estimates the extent to which changes in primary anxiety symptoms from pretreatment to posttreatment are associated with changes in the secondary outcome measures from posttreatment to follow-up. Paths $k$ and $l$ represent traditional autoregressive effects in which change in an outcome from pretreatment to posttreatment is associated with change in the same outcome from posttreatment to 12 month follow-up.

Paths $m$ and $n$ represent whether change in anxiety symptoms between pretreatment and posttreatment is associated with the initial level of secondary outcome variables (path $m$ ) and whether change in secondary outcome variables between the immediate posttreatment and the follow-up is associated with the initial level of youth anxiety symptoms (path $n$ ). Paths $o$ and $p$ represent whether change in youth anxiety symptoms between pretreatment and the immediate posttreatment is associated with the 
initial level of secondary outcome variables (path $o$ ) and whether change in youth anxiety symptoms between the immediate posttreatment and the follow-up is associated with the initial level of secondary outcome variables (path $p$ ).

Covariates. The following covariates were included in the analyses: (1) the outcomes measured at pretreatment, (2) youth's sex, (3) youth's age, and (3) comorbidity status. Paths were included from each of these variables to all endogenous variables. Figure 1 excludes the covariates and the correlations between the exogenous variables to avoid clutter, but these were included in all model tests.

Fit Indices. Following the recommendations of Bollen and Long (1993), a variety of global fit indices were used, including indices of absolute fit, indices of relative fit and indices of fit with a penalty function for lack of parsimony. These include the traditional overall chi square test of model fit, which should be statistically non-significant, the Root Mean Square Error of Approximation (RMSEA), which should be less than .08 to declare satisfactory fit), the $p$ value for the test of close fit, which should be statistically nonsignificant, and the Comparative Fit Index (CFI), which should be greater than .95 .

In addition to the global fit indices, more focused tests of fit were pursued. These included the standardized residual covariances, which should be between -2.00 and 2.00, and modification indices, which should be less than 4.00. The parameter estimates also were examined for Heywood cases. Heywood cases are parameter estimates with illogical values, such as negative error variances or intercorrelation values with absolute values greater than 1.0. Care was taken to ensure there was no specification error. Table 2 presents the fit indices for the study's SEM analyses. 
Clustering Effects. The GCBT approach consisted of 19 separate treatment groups of youths in blocks of seven. As a result, the model was adjusted for potential clustering effects (19 clusters). Participants in CBT/P were treated as independent from each other and each participant was coded as a separated cluster (100 clusters). There were a total of 119 clusters. Given that traditional regression approaches assume independence of observations, intra-class correlation (ICC) coefficients were calculated to examine the degree of non-independence of observations as a result of the clustering of participants in GCBT. This calculation is important because high ICC inflate the probability for a Type I error rate (Blair, Higgins, Topping, \& Mortimer, 1983). ICCs of zero or near zero are not a concern. Calculation of ICCs revealed coefficients greater than .05. These coefficients were large enough to warrant adjusting for clustering effects attributable to GCBT using the CLUSTER command in MPlus.

2.4.1. Mediation of Change. In terms of mediation, the joint significance test was used to test for significant effects (MacKinnon, Lockwood, Hoffman, West, \& Sheets, 2002). Figure 1 represents the model that was tested in which changes in primary anxiety symptoms were hypothesized to mediate changes in secondary, non-targeted, depressive symptoms. This model yielded a good fit to the data $(\chi 2(1)=.51, p>.05 ;$ RMSEA $<$ $.001, p=.57 ; \mathrm{CFI}=1.0 ; \mathrm{SRMR}=.007)$. More focused tests of fit revealed no theoretically meaningful or sizable modification indices. There were no absolute standardized residuals larger than 1.96. The variables in the model were able to account for $44 \%$ of the variance in the CDI posttreatment scores, and $55 \%$ of the variance in the CDI follow-up scores. 
Paths $c$ and $j$ in Figure 1 need to be statistically significant to conclude that positive changes in primary anxiety symptoms at posttreatment mediate changes in secondary, non-targeted, depressive symptoms at 12 month follow-up. Paths $k$ and $h$ in Figure 1 need to be statistically significant to conclude that positive changes in primary, targeted, anxiety symptoms at 12 month follow-up mediate changes in secondary, nontargeted, problems at 12 month follow-up. Paths $c$ and $g$ were both statistically significant (path $c: B=0.42, S E=.08, p<.001 ; 95 \% \mathrm{CI}=.26$ to .57 ; path $g: B=.52, S E=.08, p<$ $.001 ; 95 \% \mathrm{CI}=37$ to .68$)$, suggesting that positive changes in primary, targeted, anxiety symptoms at posttreatment significantly mediate positive changes in secondary, nontargeted, depressive symptoms, measured by youth rated CDI at posttreatment (see Figure 2). Paths $k$ and $h$ were statistically significant (path $k: B=0.37, S E=0.08, p<$ $.001,95 \% \mathrm{CI}=0.23$ to 0.52 ; path $h: B=0.45, S E=0.14, p=0.001,95 \% \mathrm{CI}=0.21$ to 0.72), suggesting that positive changes in primary, targeted, anxiety symptoms at 12 month follow-up significantly mediate changes in secondary, non-targeted, depressive symptoms, measured by youth rated CDI at 12 month follow-up (see Figure 2).

Additional analyses were conducted in which changes in social anxiety symptoms were hypothesized to mediate changes in depressive symptoms (see Figure 5). This model yielded a good fit to the data $(\chi 2(11)=12.35, p>.05 ;$ RMSEA $<.03, p=.69$; CFI $=.99 ; \mathrm{SRMR}=.03)$. More focused tests of fit revealed no theoretically meaningful or sizable modification indices. There were no absolute standardized residuals larger than 1.96. The variables in the model were able to account for $31 \%$ of the variance in the CDI posttreatment scores, and $38 \%$ of the variance in the CDI follow-up scores. Paths $c$ and $g$ in Figure 5 were both statistically significant (path $c: B=0.46, S E=.06, p<.001 ; 95 \%$ 
$\mathrm{CI}=.37$ to .55 ; path $g: B=.29, S E=.11, p<.001 ; 95 \% \mathrm{CI}=.07$ to .51$)$, suggesting that positive changes in social anxiety symptoms at posttreatment significantly mediate positive changes in depressive symptoms at posttreatment. Paths $k$ and $h$ in Figure 5 were statistically significant (path $k: B=0.35, S E=0.10, p<.001,95 \% \mathrm{CI}=0.23$ to 0.52 ; path $h: B=0.34, S E=0.14, p=0.01,95 \% \mathrm{CI}=0.07$ to 0.60$)$, suggesting that positive changes in social anxiety symptoms at 12 month follow-up significantly mediate changes in depressive symptoms at 12 month follow-up.

\subsubsection{Directionality of Change. The fourth aim of the present study was to} examine the directionality of change in the relation between positive changes in youth primary, targeted, anxiety symptoms and youth secondary, non-targeted, problems. Of interest are paths $g$ through $l$ in Figure 1. Again, paths $k$ and $l$ represent traditional autoregressive effects in which changes in primary and secondary outcome measures from pretreatment to posttreatment are associated with changes in the respective outcome measures from posttreatment to follow-up. Paths $g$ and $h$ represent contemporaneous reciprocal causality, in that (a) changes in youth anxiety are assumed to outcome changes in depressive symptoms, and (b) changes in depressive symptoms are assumed to outcome changes in youth anxiety.

The values of these reciprocal paths cannot be estimated from the data because this portion of the model is statistically underidentified. We therefore analyzed the model twice: once where paths $g$ and $h$ flowed from youth anxiety to depressive symptoms and a second time where paths $g$ and $h$ flowed from youth depressive to anxiety symptoms. Paths $i$ and $j$ represent lagged effects. Path $i$ estimates the extent to which changes in secondary outcome measures from pretreatment to posttreatment are associated with 
changes in primary anxiety symptoms from posttreatment to 12 month follow-up. Paths $j$ estimates the extent to which changes in primary anxiety symptoms from pretreatment to posttreatment are associated with changes in the secondary outcome measures from posttreatment to 12 month follow-up.

The directionality of change was first examined using youth RCMAS and CDI measures. The causal flow of paths $g$ at posttreatment and $h$ at 12 month follow-up was demonstrated by the previous findings. Paths $k$ and $l$ were statistically significant, suggesting that there were significant changes in social anxiety and depressive symptoms from posttreatment to 12 month follow-up (see Figure 3). In the second run, when the causal direction was reversed, paths $e$ and $g$ (path $e: B=0.34, S E=0.09, p<.001,95 \%$ $\mathrm{CI}=0.17$ to 0.51 ; path $g: B=0.48, S E=0.08, p<0.001,96 \% \mathrm{CI}=0.33$ to 0.63$)$ and paths $l$ and $h$ were also statistically significant (path $l: B=0.49 S E=0.10, p<.001,95 \%$ $\mathrm{CI}=0.28$ to 0.69 ; path $h: B=0.48, S E=0.08, p<0.001,95 \% \mathrm{CI}=0.09$ to 0.39 ) (see Figure 3).

In both runs, the general hypothesis being tested is whether changes in youth primary, targeted, anxiety symptoms are associated with changes in youth secondary, non-targeted, depressive symptoms contemporaneously; which direction(s) the causal arrow should take is ambiguous because the two models are "redundant" in SEM terminology (Silverman et al., 2009). The significance of the paths $g$ and $h$ does not provide an answer about the directionality of change between youth primary, targeted, anxiety symptoms and youth secondary, non-targeted, depressive symptoms. Similar to Silverman et al. (2009), it was assumed that the conclusions regarding the lagged effects are the same irrespective of the way the model is represented in terms of paths $g$ and $h$. 
To provide an answer about the directionality of change between primary and secondary outcome variables, it is important to consider lagged effects (paths $i$ and $j$ in Figure 1). Paths $i$ and $j$ need to be statistically significant to conclude that changes in primary, targeted, anxiety symptoms and secondary, non-targeted, depressive symptoms account for bidirectional changes in each other. Such lagged effects make theoretical sense because time must pass in order for changes in anxiety symptoms to influence changes in depressive symptoms and for changes in depressive symptoms to influence changes in anxiety symptoms. The results revealed that paths $i$ and $j$ were not statistically significant (path $i: B=0.05, S E=0.12, p>.05,95 \% \mathrm{CI}=-0.18$ to 0.28 ; path $j: B=-$ $0.02, S E=.12, p>.05,95 \% \mathrm{CI}=-0.25$ to 0.20 ) (see Figure 3 ). Changes in primary anxiety symptoms and secondary externalizing problem behaviors do not account for bidirectional changes in each other.

The directionality of change was also examined using the MASC Social subscale for social anxiety symptoms and the CDI for depressive symptoms. Similar to the previous analyses, paths $c$ and $g$ were both statistically significant suggesting that positive changes in social anxiety symptoms at posttreatment significantly outcome changes in secondary, non-targeted, depressive symptoms at posttreatment. Paths $k$ and $h$ were statistically significant, suggesting that positive changes in social anxiety symptoms at 12 month follow-up significantly outcome changes in depressive symptoms over the same period of time.

In the second run, paths $e$ and $g$ at posttretament were statistically significant (path $e: B=0.40, S E=0.09, p<.001,95 \% \mathrm{CI}=0.22$ to 0.57 ; path $g: B=0.23, S E=0.10$, $p<0.01,96 \% \mathrm{CI}=0.05$ to 0.42 ). In the second run, paths $l$ and $h$ were statistically 
significant (path $l: B=0.41 S E=0.11, p<.001,95 \% \mathrm{CI}=0.19$ to 0.60 ; path $h: B=0.30$, $S E=0.13, p>0.05,95 \% \mathrm{CI}=0.06$ to 0.55 ) (see Figure 6). In terms of lagged effects, path $i$ was statistically significant (path $i: B=0.20, S E=0.10, p<.05,95 \% \mathrm{CI}=-0.18$ to 0.28). Path $j$, however, was not statistically significant (path $j: B=-0.05, S E=.15, p>$ $.05,95 \% \mathrm{CI}=-0.33$ to 0.24 ) (see Figure 6). The significance of path $i$ suggests that changes in youth depressive symptoms outcome changes in social anxiety rather than vice versa. Path $i$ is a lagged effect by which it takes time for reductions in youth depressive symptoms to work their way through to result in changes in social anxiety symptoms.

2.6.1. Moderated Mediation. The fifth specific aim within the second objective was to evaluate whether the hypothesized mediated and bidirectional relations between changes in primary anxiety symptoms and secondary outcome problems are moderated by treatment approach (CBT/P versus GCBT) and youth characteristics (sex and age). This aim was pursued using a product term approach for continuous moderators (i.e., age) and a multiple group approach for categorical moderators (i.e., treatment approach and sex) (Jaccard \& Wan, 1996; Jaccard, Turrisi, \& Wan, 1990). The product term approach was explained earlier.

The multiple group approach involved running a given model twice - once with the path coefficients constrained equal across the two levels of a given moderator (e.g., treatment approach; CBT/P and GCBT) and once with the path coefficients unconstrained. A chi square difference test was calculated next by subtracting the chi square value and degrees of freedom of the constrained and unconstrained models. The statistical significance of the chi square difference was determined by comparing the 
observed value against a critical value found in a chi square table. The chi square difference needs to be statistically significant to conclude that the mediated and bidirectional relations were moderated by a given categorical variable.

Treatment Approach. A multiple group approach was pursued to test the moderating effect of treatment approach on the contemporaneous effects from anxiety to depressive symptoms, the lagged effect from anxiety symptoms at posttreatment to depressive symptoms at 12 month follow-up, and the lagged effect from depressive symptoms at posttreatment to anxiety symptoms at 12 month follow-up (paths $g$ through $j$, in Figure 1). The chi square test of model fit for the unconstrained model in which paths $g$ through $j$ in Figure 1 were allowed to vary across CBT/P and GCBT was $\left(\chi^{2}\right.$ (6) $=14.03, p>.05)$. The chi square test of model fit for the unconstrained model in which paths $g$ through $j$ in Figure 1 were hypothesized to vary between CBT/P and GCBT was $(\chi 2(2)=.22, p>.05)$. The chi square difference test between the constrained and unconstrained model was statistically significant, $\chi^{2}$ diff. $(2)=10.30, p<.05$. The results suggest that the unconstrained model for which the paths coefficients were allowed to vary between $\mathrm{CBT} / \mathrm{P}$ and GCBT provided a relatively better fit than the constrained model for which the paths were hypothesized to be equal.

Relaxing paths $g$ through $j$ one by one revealed that the contemporaneous paths $h$ flowing from anxiety to depressive symptoms was moderated by treatment approach (path $h: \mathrm{CBT} / \mathrm{P}=.82, S E=.18, p<.001$; GCBT: $B=.34, S E=14, p=.01$ ) (see Figure 4). Changes in anxiety symptoms from posttreatment to 12 month follow-up mediated changes in depressive symptoms over the same time period and these changes were stronger in $\mathrm{CBT} / \mathrm{P}$ relative to GCBT. 
The analyses were repeated using youth MASC Social and CDI measures. The chi square test of model fit for the unconstrained model in which paths $g$ through $j$ in Figure 1 were allowed to vary across CBT/P and GCBT was $\left(\chi^{2}(9)=11.94, p>.05\right)$. The chi square test of model fit for the constrained model in which paths $g$ through $j$ in Figure 1 were hypothesized to be equal for CBT/P and GCBT was $(\chi 2(7)=17.25, p>.05)$. The chi square difference test between the constrained and unconstrained model was statistically significant, $\chi^{2}$ diff. $(1)=5.31, p<.05$. The results suggest that the unconstrained model for which the paths coefficients were allowed to vary between $\mathrm{CBT} / \mathrm{P}$ and GCBT provided a relatively better fit than the constrained model for which the paths were hypothesized to be equal.

Relaxing paths $g$ through $j$ one by one revealed that treatment approach moderated the contemporaneous paths $g$ and $h$ flowing from social anxiety to depressive symptoms at posttreatment and 12 month follow-up, respectively. Path $g$ at posttreatment was statistically significant in GCBT $(B=0.60, S E=0.10, p<.001)$ but not in $\mathrm{CBT} / \mathrm{P}(B$ $=0.07, S E=0.14, p>.05)$ (see Figure 7). The nature of the interaction appears to reflect a difference in the fundamental mediated relation between social anxiety and depressive symptoms: Changes in social anxiety symptoms from pretreatment to posttreatment mediated changes in depressive symptoms over the same time period in GCBT but not $\mathrm{CBT} / \mathrm{P}$.

Path $g$ at 12 month follow-up was statistically significant in CBT/P $(B=0.60, S E$ $=0.14, p<.001)$ but not in GCBT $(B=0.58, S E=0.13, p>.05)$ (see Figure 7). Changes in social anxiety symptoms from posttreatment to 12 month follow-up mediate changes in depressive symptoms over the same time period in CBT/P but not in GCBT. Although 
not a moderated path per se, inspection of individual path coefficients for the two treatment approaches revealed that path $i$ was statistically significant in $\mathrm{CBT} / \mathrm{P}(B=0.32$, $S E=0.14, p=.02)$ but not $\operatorname{GCBT}(B=0.21, S E=0.12, p>.05)$ (see Figure 7$)$. This suggests that in $\mathrm{CBT} / \mathrm{P}$ the flow of change is from youth depressive to social anxiety symptoms rather than vice versa.

Youth Sex. A multiple group approach was pursued to test the moderating effect of youth sex on the contemporaneous effects from anxiety to depressive symptoms, the lagged effect from anxiety symptoms at posttreatment to depressive symptoms at 12 month follow-up, and the lagged effect from depressive symptoms at posttreatment to anxiety symptoms at 12 month follow-up (paths $g$ through $j$ in Figure 1). A dummy variable was created to indicate boys $(n=98)$ and girls $(n=85)$.

The chi square test of model fit for the unconstrained model in which paths $g$ through $j$ in Figure 1 were allowed to vary for boys and girls was $\left(\chi^{2}(1)=.00, p<.05\right)$. The chi square test of model fit for the constrained model in which paths $g$ and path $j$ in Figure 1 was hypothesized to be equal for boys and girls was $(\chi 2(2)=.25, p>.05)$. The chi square difference test between the constrained and unconstrained model was not statistically significant, $\chi 2$ diff. $(1)=.25, p>.05$. The non-significant chi square difference suggests a lack of a significant interaction effect of youth sex on paths $g$ through $j$ in Figure 1. The results suggest that the mediated relations between changes in anxiety and depressive symptoms are not moderated by youth sex.

The above analysis was repeated using youth MASC Social and CDI measures. The chi square test of model fit for the unconstrained model was $\left(\chi^{2}(20)=19.77, p>\right.$ $.05)$. The chi square test of model fit for the constrained model was $\left(\chi^{2}(21)=24.70, p>\right.$ 
$.05)$. The chi square difference test between the constrained and unconstrained model was statistically significant, $\chi^{2}$ diff. $(1)=4.09, p<.05$. The results suggest that the unconstrained model for which the paths for boys and girls were allowed to vary provided a relatively better fit than the unconstrained model for which the paths were hypothesized to be equal for boys and girls.

Relaxing paths $g$ through $j$ one by one revealed youth sex moderated the lagged effect from youth depressive symptoms at posttreatment to youth social anxiety symptoms at 12 month follow-up. Path $i$ was statistically significant for girls $(B=0.47$, $S E=0.16, p=.01)$ but not for boys $(B=0.13, S E=0.08, p>.05)$ (see Figure 8). For girls, as changes in depressive symptoms from pretreatment to posttreatment increased, changes in social anxiety symptoms from posttreatment to 12 month follow-up increased. The results suggest that for girls the flow of change is from depressive to social anxiety symptoms rather than vice versa.

Although not a moderated path per se, inspection of individual path coefficients revealed that path $g$ and path $h$ were statistically significant for girls (path $g: B=.40, S E$ $=.17, p<.05$; path $h: B=.60, S E=.11, p<.001$ ) but not for boys (path $g: B=0.21, S E=$ $0.12, p>.05$; path $h: B=.24, S E=.20, p>.05$ ) (see Figure 8 ). Changes in social anxiety symptoms from pretreatment to posttreatment and from posttreatment to 12 month follow-up mediate changes in depressive symptoms over the respective period of time for girls but not for boys.

Youth Age. Figure 4 represents the model tested. The key paths of interest in Figure 4 are $r$ through $z$. Path $r$ needs to be statistically significant to conclude there is a significant interaction effect of youth age on the contemporaneous effect from anxiety 
symptoms at posttreatment onto depressive symptoms at posttreatment. Path $s$ needs to be statistically significant to conclude there is a significant interaction effect of youth age on the lagged effect from anxiety symptoms at posttreatment onto depressive symptoms at 12 month follow-up. Path $t$ needs to be statistically significant to conclude there is a significant interaction effect of youth age on the lagged effect from depressive symptoms at posttreatment onto depressive symptoms at 12 month follow-up. Path $z$ needs to be statistically significant to conclude that there is a significant interaction effect of youth age on the contemporaneous effect from anxiety symptoms onto depressive symptoms at 12 month follow-up.

The model yielded a good fit to the data. The overall chi square test of model fit was not statistically significant $(\chi 2(3)=1.80, p>.05)$. The CFI was 1.00 , the RMSEA was $<.001$, the $p$ value for the test of close fit was 0.76 , and the SRMR was .003 . More focused tests of fit revealed no theoretically meaningful or sizeable modification indices. Paths $r$ through $z$ were not statistically significant. The results suggest that the mediated relations between changes in anxiety and depressive symptoms are not moderated by youth age. In addition to the product term analyses, a multiple group approach was pursued using younger children (ages 6 to 12) and older children (ages 7 to 16) as the grouping variables. No evidence for moderation was found using this approach using youth RCMAS and CDI and youth MASC Social and CDI models. The mediated relations between changes in social anxiety and depressive symptoms were therefore not moderated by youth age. 
Objective II: Secondary Outcomes: Externalizing Problem Behaviors

1.2.Treatment Outcome. To evaluate whether 12 to 14 weeks of anxiety treatment yielded changes in externalizing problem behaviors analyses of variance were pursued in a SEM framework collapsing the outcomes across treatments. The SEM equivalent of a $2 \mathrm{X} 3$ between-within subjects analysis of variance was conducted on the parent rated CBCL-E, with the type of treatment approach (CBT/P and GCBT) representing a between-subjects factor and time (pre and post; post and follow-up) representing a within-subjects factor. In terms of changes in externalizing problem behaviors from pretreatment to posttreatment, the main effect for time on the CBCL-E was statistically significant $(\mathrm{B}=55.94, p<.001)$. Table 5 presents relevant single degree of freedom contrasts and their associated statistics for CBCL-E. The contrasts used non-pooled error terms for the contrasts involving repeated measures but pooled terms for the contrasts across the between-subjects factor.

The mean difference for the main effect of time from pretreatment to posttreatment collapsing across treatments was 5.84 with posttreatment showing a decrease in parent ratings of their child's externalizing problem behaviors, on average, than pretreatment. In terms of posttreatment to 12 month follow-up, the main effect for time on the CBCL-E was statistically significant $(B=68.53, p<.001)$. Table 4 presents relevant single degree of freedom contrasts and their associated statistics for CBCL-E. The contrasts used non-pooled error terms for the contrasts involving repeated measures but pooled terms for the contrasts across the between-subjects factor. The mean difference for the main effect of time collapsing across treatment approach was 1.27 with 
12 month follow-up showing a decrease in parents' ratings of their child's externalizing problem behaviors, on average, than posttreatment.

2.2.2. The Relative Efficacy of $C B T / P$ vs. GCBT. Of interest was whether the positive changes in secondary, non-targeted externalizing problem behaviors following treatment were statistically significantly different in $\mathrm{CBT} / \mathrm{P}$ relative to GCBT at posttreatment and 12 month follow-up. The interaction effect of treatment approach (CBT/P and GCBT) by time (pre and post) was not statistically significant $(B=-1.20, p>$ .05). The interaction effect of treatment approach (CBT/P and GCBT) by time (post and follow-up) was not statistically significant $(B=-2.17, p>.05)$.

To determine if the pretreatment to posttreatment time difference occurred for each treatment approach, simple main effects (SME) contrasts were performed. The difference was statistically significant for both treatment approaches with posttreatment scores showing a decrease in parent ratings of their child's externalizing problem behaviors, on average, than pretreatment. The time difference for CBT/P was 5.57 and for GCBT it was 6.39. The significant pretreatment to posttreatment time difference effects were maintained when the Holm modified Bonferroni method was applied to control the experiment-wise error rate at 0.05 .

To determine if the posttreatment to 12 month follow-up time difference occurred at each treatment approach, simple main effects (SME) contrasts were performed. The difference was statistically significant for both treatment approaches with 12 month follow-up scores showing a decrease in parents' ratings of their child's externalizing problem behaviors, on average, than posttreatment. The time difference for $\mathrm{CBT} / \mathrm{P}$ was .60 and for GCBT it was 2.01. The significant posttreatment to 12 month follow-up time 
difference effects were maintained when the Holm modified Bonferroni method was applied to control the experiment-wise error rate at 0.05 .

2.3.2. Change as a Function of Youth Characteristics. Of interest was whether the hypothesized positive changes in secondary externalizing problem behaviors following treatment were statistically significantly different for girls versus boys and younger versus older children. The interaction effect of youth sex and time (pre to post) was not statistically significant $(B=-.53, p>.05)$. The interaction effect of youth sex and time (post to follow-up) was not statistically significant $(B=-1.79, p>.05)$. The interaction effect of youth age and time (pre to post) was not statistically significant $(B=.65, p>$ $.05)$. The interaction effect of youth age and time (post to follow-up) was not statistically significant $(B=-.13, p>.05)$.

To determine if the pretreatment to postttreatment and posttreatment to 12 month follow-up time difference in externalizing problem behaviors occurred at each level of the moderator variables, simple main effects (SME) contrasts were performed. The pretreatment to postttreatment and posttreatment to 12 month follow-up time difference was statistically significant for boys and girls, younger (6 to 11 years) and older children (12 to 16 years). Posttreatment scores showed a decrease in youth externalizing problem behaviors relative to pretreatment. Twelve month follow-up scores showed a decrease in youth externalizing problem behaviors relative to posttreatment. The time difference effects remained significant when the Holm modified Bonferroni method was applied to control the experiment-wise error rate at 0.05 .

2.4.2. Mediation of Change. Figure 8 represents the model that was tested in which changes in primary anxiety symptoms were hypothesized to mediate changes in 
secondary externalizing problem behaviors. This model yielded a good fit to the data $(\chi 2$ $(3)=3.76, p>.05 ; \mathrm{RMSEA}<.04, p=.47 ; \mathrm{CFI}=.99 ; \mathrm{SRMR}=.02)$. More focused tests of fit revealed no theoretically meaningful or sizable modification indices. There were no absolute standardized residuals larger than 1.96. The variables in the model were able to account for $56 \%$ of the variance in the CBCL-E posttreatment scores, and $57 \%$ of the variance in the RCMAS/P follow-up scores.

Paths $c$ and $g$ were both statistically significant (path $c: B=.52, S E=.07, p<$ $.001 ; 95 \% \mathrm{CI}=.39$ to .65 ; path $g: B=.74, S E=.14, p<.001 ; 95 \% \mathrm{CI}=0.48$ to 1.00$)$, suggesting that positive changes in primary anxiety symptoms at posttreatment significantly mediate positive changes in secondary externalizing problem behaviors, measured by parent rated CBCL-E at posttreatment (see Figure 9). Paths $k$ and $h$ in Figure 2 were statistically significant (path $k: B=.43, S E=.09, p<.001,95 \% \mathrm{CI}=.25$ to .61 ; path $h: B=1.02, S E=0.15, p=0.001,95 \% \mathrm{CI}=.73$ to 1.30$)$, suggesting that positive changes in anxiety symptoms at 12 month follow-up significantly mediate changes in secondary externalizing problem behaviors, measured by parent rated CBCLE at 12 month follow-up (see Figure 9).

\subsubsection{Directionality of Change. Paths $i$ and $j$ in Figure 8 need to be statistically}

significant to conclude that changes in primary anxiety symptoms and secondary externalizing problems account for bidirectional changes in each other. Paths $i$ and $j$ were not statistically significant (path $i: B=.08, S E=.05, p>.05,95 \% \mathrm{CI}=-.01$ to .18 ; path $j: B=.01, S E=$ $.16, p>.05,95 \% \mathrm{CI}=-.31$ to .33 ) (see Figure 10 ). Changes in primary anxiety symptoms and secondary externalizing problem behaviors do not account for bidirectional changes in each other. 
2.6.2. Moderated Mediation. Of interest was whether the hypothesized mediation and bidirectional relations between changes in youth primary anxiety symptoms and youth secondary externalizing problem behaviors are moderated by treatment approach (CBT/P; GCBT) and youth characteristics (sex and age).

Treatment Approach. A multiple group approach was pursued to test the moderating effect of treatment approach on the contemporaneous effects from anxiety symptoms to externalizing problem behaviors, the lagged effect from anxiety symptoms at posttreatment to externalizing problem behaviors at 12 month follow-up, and the lagged effect from externalizing problem behaviors at posttreatment to anxiety symptoms at 12 month follow-up (paths $g$ through $j$, in Figure 1). The chi square test of model fit for the unconstrained model in which paths $g$ through $j$ in Figure 1 were allowed to vary across CBT/P and GCBT was $(\chi 2(0)=.00, p<.05)$. The chi square test of model fit for the unconstrained model in which paths $g$ through $j$ in Figure 1 were hypothesized to vary between $\mathrm{CBT} / \mathrm{P}$ and GCBT was $(\chi 2(3)=1.28, p>.05)$. The chi square difference test between the constrained and unconstrained model was not statistically significant, $\chi 2$ diff. $(3)=1.28, p>.05$. The hypothesized mediated and bidirectional relations between changes in anxiety symptoms and externalizing problem behaviors were therefore not moderated by treatment approach.

Youth Sex. A multiple group approach was pursued to assess the moderating effect of youth sex on the contemporaneous effects from anxiety symptoms to externalizing problem behaviors, the lagged effect from anxiety symptoms at posttreatment to externalizing problem behaviors at 12 month follow-up, and the lagged effect from externalizing problem behaviors at posttreatment to anxiety symptoms at 12 
month follow-up (paths $g$ through $j$ in Figure 1). A multiple group model in which paths $g$ through $j$ were constrained to be equal for boys and girls yielded $\left(\chi^{2}(21)=11.41, p>\right.$ .05). A multiple group model for which paths $g$ through $j$ were allowed to vary between boys and girls yielded $(\chi 2(20)=10.80, p>.05)$. The chi square difference test between the constrained and unconstrained model was statistically significant, $\chi 2$ diff. $(1)=.61, p$ $>.05$. The hypothesized mediated and bidirectional relations between changes in anxiety symptoms and externalizing problem behaviors were therefore not moderated by youth sex.

Youth Age. Figure 11 represents the model tested. The key paths of interest in Figure 4 are $r$ through $z$. The model in Figure 7 yielded a good fit to the data. The overall chi square test of model fit was not statistically significant $(\chi 2(3)=1.80, p>.05)$. The CFI was 1.00 , the RMSEA was $<.001$, the $p$ value for the test of close fit was 0.76 , and the SRMR was .003. More focused tests of fit revealed no theoretically meaningful or sizeable modification indices. Path $r$ was not statistically significant $(p>.05)$, suggesting a lack of significant interaction effects of youth age on the lagged effect from primary anxiety symptoms at posttreatment to secondary externalizing problem behaviors at 12 month follow-up. Path $s$ was not statistically significant $(p>.05)$, suggesting a lack of significant interaction effect of youth age on the lagged effect from secondary externalizing problem behaviors at posttreatment to primary anxiety symptoms at 12 month follow-up. The hypothesized mediation and bidirectional relations between changes in youth primary anxiety symptoms and youth secondary externalizing problem behaviors are not moderated by youth age. 


\section{CHAPTER V \\ DISCUSSION}

The present study pursued two objectives in the context of a randomized clinical trial of cognitive-behavioral therapy with parent (CBT/P) and group (GCBT)

involvement. The first objective was to examine the variability in treatment outcome. There were three specific aims within the first objective, to evaluate: (1) youth characteristics (age, depressive, and externalizing disorders) as moderators of treatment outcome; (2) the differential outcome of the treatment approaches as a function of youth characteristics; and (3) the relative efficacy of the treatment approaches at each level of the moderators.

The second objective was to evaluate the efficacy of anxiety treatments along secondary depressive symptoms and externalizing behaviors. There were five specific aims within the second objective, to evaluate: (1) whether anxiety treatment yields reductions in secondary problems, (2) the efficacy of anxiety treatments in reducing secondary problems as a function of approach and youth characteristics, (3) whether reductions in anxiety symptoms significantly mediate changes in secondary problems, (4) the directionality of change in the hypothesized mediated relations, and (5) whether the hypothesized mediated relations are moderated by treatment approach and youth characteristics.

\section{Summary of Dissertation Findings}

In terms of the present study's first objective, the findings revealed that youth age, comorbidity with depressive disorders, and comorbidity with externalizing disorders were significant moderators of posttreatment outcome using parent but not youth report. 
$\mathrm{CBT} / \mathrm{P}$ relative to GCBT lowered anxiety symptoms more for younger than older children. GCBT relative to CBT/P lowered anxiety symptoms more for older than younger children. In terms of comorbidity with depressive disorders, GCBT relative to $\mathrm{CBT} / \mathrm{P}$ lowered anxiety symptoms more for youth with comorbid depressive disorders than youth without comorbid depressive disorders. In terms of comorbidity with externalizing disorders, GCBT relative to $\mathrm{CBT} / \mathrm{P}$ lowered anxiety symptoms less for youth with comorbid externalizing disorders than youth without comorbid externalizing disorders. Youth age, comorbidity with depressive disorders, and comorbidity with externalizing disorders were not significant moderators of 12 month follow-up outcome using youth and parent report.

In terms of the present study's second objective, the findings revealed that there were statistically significant reductions in secondary, non-targeted, depressive symptoms and externalizing problem behaviors at both posttreatment and 12 month follow-up. In terms of relative efficacy, reductions in secondary depressive symptoms and externalizing problem behaviors were not statistically significantly different in CBT/P relative to GCBT. Reductions in secondary, non-targeted, depressive symptoms and externalizing problem behaviors occurred in both treatments and were of relative strength. In terms of change as a function of youth characteristics, there were statistically significant reductions in depressive symptoms and externalizing problem behaviors from pretreatment to posttreatment, as well as from posttreatment to 12 month follow-up for boys and girls and irrespective of youth age.

In terms of mediation, the findings revealed that reductions in secondary, nontargeted, depressive symptoms and externalizing problem behaviors were mediated by 
reductions in anxiety symptoms. Treatment yielded significant reductions in targeted anxiety symptoms at posttreatment and 12 month follow-up. Reductions in targeted anxiety symptoms at posttreatment and 12 month follow-up, in turn, significantly reduced non-targeted depressive symptoms and externalizing problem behaviors at the respective periods of time. Reductions in secondary, non-targeted, depressive symptoms were also mediated by reductions in social anxiety symptoms.

In terms of directionality of change, there was evidence for reversed directionality when the variables of interest were social anxiety and depressive symptoms. The lagged path that linked changes in depressive symptoms to subsequent changes in social anxiety symptoms was statistically significant. The lagged path that linked changes in social anxiety to subsequent changes in depressive symptoms was not significant. These findings suggest that the direction of change was from youth depressive to social anxiety symptoms rather than vice versa. The directionality of change, however, was qualified or moderated by treatment approach and youth sex as further discussed below.

There was no evidence for reversed directionality when the variables of interest were youth general anxiety and depressive symptoms, nor was there evidence for reversed directionality when the variables of interest were youth general anxiety symptoms and externalizing problem behaviors. The lagged path that linked changes in youth general anxiety to subsequent changes in depressive symptoms was not significant, nor was the lagged path that linked changes in youth general anxiety symptoms to subsequent changes in externalizing problem behaviors. Similarly, the lagged path that linked changes in youth depressive to subsequent changes in general anxiety symptoms was not statistically significant, nor was the lagged effect that linked changes in youth 
externalizing problem behaviors to subsequent changes in general anxiety symptoms. These findings suggest that the direction of change was from youth general anxiety to depressive symptoms and from youth general anxiety to externalizing problem behaviors rather than vice versa.

In terms of moderated mediation, the mediated relations between reductions in youth general anxiety and depressive symptoms were moderated by treatment approach. The nature of the interaction was one of relative strength rather than a difference in the fundamental mediated relation between reductions in youth general anxiety and depressive symptoms. Reductions in youth general anxiety symptoms from posttreatment to 12 month follow-up mediated reductions in depressive symptoms over the same time period and this relation was stronger in $\mathrm{CBT} / \mathrm{P}$ relative to $\mathrm{GCBT}$.

The mediated relations between reductions in social anxiety and depressive symptoms were moderated by treatment approach and youth sex. The nature of the interaction reflected a difference in the fundamental mediated relation between reductions in youth social anxiety and depressive symptoms rather than a difference in relative strength. In terms of treatment approach, at posttreatment, reductions in social anxiety mediated changes in depressive symptoms in GCBT but not CBT/P. Reductions in depressive symptoms from pretreatment to posttreatment mediated subsequent changes in social anxiety symptoms at 12 month follow-up in CBT/P but not in GCBT. Reductions in social anxiety symptoms from posttreatment to 12 month follow-up mediated changes in depressive symptoms over the same period of time in CBT/P but not GCBT. These moderated mediation findings suggests that in $\mathrm{CBT} / \mathrm{P}$ the direction of change was from 
depressive to social anxiety symptoms; in GCBT the direction of change was from social anxiety to depressive symptoms.

In terms of youth sex, the treatments significantly reduced depressive symptoms for boys and girls. Reductions in social anxiety mediated changes in depressive symptoms for girls but not boys at posttreatment and 12 month follow-up. Also for girls, reductions in depressive symptoms at posttreatment mediated subsequent changes in social anxiety symptoms at 12 month follow-up.

\section{Contributions and Implications}

The present study contributes on theoretical, empirical and clinical grounds. The main contributions on each of these grounds are summarized below including potential implications.

Theoretical Contributions. In terms of theoretical contributions, knowledge of treatment moderators that emerged from the present study can inform understanding of psychopathology (e.g., Kazdin, 2001; Kraermer et al., 2002). That CBT/P relative to GCBT lowered anxiety symptoms more for younger children than older children is consistent with developmental theory which states that parents are most likely to play an influential role in the lives of young children (e.g., Daleiden et al., 1999; Hudson et al., 2002). It is therefore interesting that $\mathrm{CBT} / \mathrm{P}$, a treatment program that targeted parenting skills and parent-youth relationships, was particularly efficacious for younger children. That GCBT relative to CBT/P lowered anxiety symptoms more for older children than younger children is also consistent with developmental theory and research showing that difficulties with social skills and problematic peer-youth relationships are most likely to be recognized in adolescence (Parker et al., 2006). It is also in adolescence that the social 
skills involved in effective peer-youth relationships become increasingly complex. It is therefore interesting that GCBT, a treatment program that targeted social skills and peeryouth relationships, was particularly effective for older children.

In terms of comorbidity, GCBT relative to $\mathrm{CBT} / \mathrm{P}$ lowered anxiety symptoms more for youth with comorbid depressive disorders than youth without comorbid depressive disorders. Put differently, CBT/P relative to GCBT lowered anxiety symptoms less for youth with comorbid depressive disorders than youth without comorbid depressive disorders. Finding that comorbid depressive disorders moderated treatment outcome is somewhat consistent with an earlier study in which comorbidity with depressive disorders was also of interest (Berman et al., 2001). Participants in that study were youth who enrolled in one of two RCTs on phobic and anxiety disorders in youth (Silverman, et al., 1999a; Silverman et al., 1999b). Participants received an exposure based treatment that involved the use of CBT in either an individual or group treatment approach. Adverse effects were found for the presence of depressive disorders for the overall sample. Separate analyses by treatment approach, however, revealed that it was the individual not the group treatment approach that accounted for the findings.

It therefore appears that in both Berman et al. (2000) and the present study, the presence of comorbid disorders did not have adverse effects when youth were assigned to a group treatment. In the present study, GCBT relative to $\mathrm{CBT} / \mathrm{P}$ lowered anxiety symptoms more for youth with comorbid depressive disorders than youth without comorbid depressive disorders. The training in social skills and peer-youth relationships delivered in GCBT may have played an important role in anxiety symptom reduction particularly for anxious youth with comorbid depressive disorders. That GCBT relative to 
CBT/P lowered anxiety symptoms more for youth with comorbid depressive disorders than youth without comorbid depressive disorders is consistent with psychopathology research showing that youth with depressive symptoms have social skills deficits and difficulties with peer-youth relationships (e.g., Joiner et al., 1999; Lewinsohn, 1974).

The findings also showed that GCBT relative to CBT/P lowered anxiety symptoms less for youth with comorbid externalizing disorders than youth without comorbid externalizing disorders. These findings are best understood using the transfer of control model (Silverman \& Kurtines, 1996). It is possible that a block in the transfer of control over the skills for improving anxiety occurred in GCBT due to youths' inability to regulate their own externalizing behaviors. This block in the transfer of control over the skills for improving anxiety may be especially salient for youth with comorbid externalizing disorders than youth without comorbid externalizing disorders. The former may find it relatively difficult to regulate their behaviors in ways that interfere with anxiety symptom reduction.

It is possible that a block in the transfer of control over the skills for improving anxiety occurred in GCBT also because this approach did not equip parents with skills to successfully manage their child's behaviors. In the absence of parent training, GCBT relies on the child's own abilities to regulate not only anxiety but also externalizing problem behaviors. It is possible that a parallel block in the transfer of control did not occur in CBT/P because this approach did equip parents with skills to successfully manage not only child avoidance but perhaps other behaviors, as well, such as externalizing problems. The relative block in the transfer of control over the skills for improving anxiety may therefore explain why GCBT compared to CBT/P lowered 
anxiety symptoms less for youth with comorbid externalizing disorders than youth without comorbid externalizing disorders.

Knowledge of treatment moderators that emerged from the present study also illustrates the importance of time and timing in therapeutic change (e.g., Granic, 2006; Silverman et al., 2009). It also identifies subgroups of youth with different course of illness or therapeutic change (e.g., Kraemer et al., 2002). Indeed, it is interesting that youth age and comorbidity impacted treatment outcome at posttreatment but not at 12 month follow-up. One explanation for the null findings at 12 month follow-up is that by then any significant interaction effects wear off. One possibility is that improvements in anxiety symptoms from pretreatment to posttreatment among youth with comorbid depressive disorders in GCBT and youth with comorbid externalizing disorders in CBT/P may wane between posttreatment and 12 month follow-up. Alternatively, perhaps by the follow-up youth with comorbid depressive disorders in CBT/P "catch up" in anxiety symptom reduction with youth without comorbid depressive disorders in $\mathrm{CBT} / \mathrm{P}$, as well as youth with comorbid depressive disorders in GCBT. Perhaps by the follow-up youth with comorbid externalizing disorders in GCBT "catch up" in anxiety symptom reduction with youth without comorbid depressive disorders in GCBT, as well as youth with comorbid externalizing disorders in $\mathrm{CBT} / \mathrm{P}$.

Indeed, if treatment impacts comorbidity, by 12 month follow-up anxiety, depressive and externalizing problems improve, leaving little, if any variability, in both treatment moderators and outcomes to detect interaction and simple main effects. This possibility is supported by the secondary outcome findings showing that anxiety, depressive and externalizing problems improved over time. It therefore appears that not 
only does comorbidity impact treatment outcome, but treatment also impacts comorbidity. Because treatment moderation was found based on parent but not youth report, it is possible that youths' and parents' perceptions of treatment outcome become increasingly attuned with each other over time, which may be another reason why the interaction effects were no longer significant at 12 month follow-up.

Secondary Outcomes. Knowledge about mediation and directionality of change that emerged in the present study could also extend understanding of psychopathology with respect to change (Howe et al., 2002). The mediation findings, in particular, advance theoretical understanding about the processes by which treatment approaches for youth anxiety and its disorders produce changes in youths' depressive and externalizing problems. This understanding is important because knowledge about mechanisms and theories of change in child psychotherapy is presently scarce (Kazdin, 2007).

Knowledge about moderated mediation that emerged in the present study also extends understanding of psychopathology with respect to maintenance and change (Howe et al., 2002). Indeed, recall that the mediated relations between changes in social anxiety and depressive symptoms were moderated by treatment approach and youth sex. In terms of treatment approaches, reductions in social anxiety symptoms mediated changes in depressive symptoms at posttreatment in GCBT but not CBT/P. The opposite was true at 12 month follow-up when reductions in social anxiety mediated changes in depressive symptoms in CBT/P but not GCBT.

In terms of youth sex, reductions in social anxiety symptoms at posttreatment and 12 month follow-up mediated changes in depressive symptoms over the respective periods of time for girls but not boys. Similarly, changes in depressive symptoms at 
posttreatment mediated subsequent reductions in social anxiety symptoms at 12 month follow-up for girls but not boys.

One possible theoretical implication of the moderated mediation by treatment approach is that treatment may have an impact on psychopathology in different subgroups, but different approaches show diverse patterns of mediation (e.g., Howe et al., 2002). Different change processes in the relation between social anxiety and depressive symptoms may operate in different approaches. Indeed, although changes in social anxiety symptoms from pretreatment to posttreatment occurred in both CBT/P and GCBT, changes in social anxiety symptoms mediated reductions in depressive symptoms over the same period of time only in GCBT. The moderated mediation by treatment approach suggests that improvements in social anxiety from pretreatment to posttreatment generalized in improvements in depressive symptoms over the same period of time only in GCBT. That changes in social anxiety symptoms from pretreatment to posttreatment did not mediate reductions in depressive symptoms in $\mathrm{CBT} / \mathrm{P}$ suggests that in this treatment approach changes in depressive symptoms occurred through other mechanisms -- one being changes in youth general anxiety symptoms.

In terms of directionality of change, the presence of reversed directionality in $\mathrm{CBT} / \mathrm{P}$ suggests that the direction of change in treatment approach was from youth depressive to social anxiety symptoms. This finding is important because social anxiety and depressive symptoms are frequently co-occurring. This finding is also important because socially phobic youth tend to not benefit from CBT when they are clinically depressed (Crawley, Beidas, Benjamin, Martin, \& Kendall, 2008). This finding suggests 
that in CBT/P depressive symptoms likely maintain social anxiety and reductions in depressive symptoms account for changes in social anxiety.

The absence of reversed directionality in GCBT suggests that the direction of change in GCBT was from social anxiety to depressive symptoms. Finding that the direction of change in GCBT was from social anxiety to depressive symptoms is consistent with an earlier study examining changes in social anxiety and depressive symptoms during group treatment for adults with SOP (Moscovitch, Hofmann, Suvak, \& In-Albon, 2005). In that study, reductions in social anxiety mediated changes in depressive symptoms, but the reverse relation was not found. Group treatment is repeatedly argued in the literature to offer opportunities to reduce social anxiety and improve social skills in children with SOP (Beidel et al., 2000; Spence et al., 2000). Support for reduced social anxiety and improved social skills was found in group based CBT programs with social skills training (Beidel et al., 2000; Spence et al., 2000). That GCBT reduced social anxiety symptoms from pretreatment to posttreatment and these reductions mediated changes in depressive symptoms over the same period of time makes theoretical sense because socially anxious and depressed youth encounter difficulties with social skills and peer-youth relationships (e.g., Joiner et al., 1999; Lewinsohn, 1974). Both of these difficulties were targeted in GCBT.

From a developmental psychopathology perspective (Cicchetti \& Rogosch 1996; Cicchetti \& Toth, 2009), the differential pattern of mediation and directionality of change by treatment approach suggests that the principle of multifinality may be at play when it comes to understanding the relation between social anxiety and depressive symptoms. Multifinality refers to the notion that a given risk factor may lead to different outcomes. 
The principle of multifinality is relevant to the present discussion because social anxiety and depression are not only highly comorbid, but also have many similar interpersonal risk factors, such as social skills deficits and problematic peer-youth relationships (e.g., Epkins \& Heckler, 2011).

The differential pattern of mediation and directionality by treatment approach also suggests that the principle of equifinality may be at play when it comes to understanding the maintenance and change of psychopathology (Howe et al., 2002). Equifinality refers to the notion that a given end state can be reached by many potential means. That the direction of change in $\mathrm{CBT} / \mathrm{P}$ was from youth depressive to social anxiety symptoms suggests that depressive symptoms maintained social anxiety in this treatment approach. It also suggests that in this treatment approach it takes time for reductions in youth depressive symptoms to work their way through to result in changes in social anxiety. Indeed, in $\mathrm{CBT} / \mathrm{P}$, it was not until reductions in youth depressive symptoms worked their way through to result in changes in social anxiety symptoms that changes in social anxiety symptoms mediated reductions in depressive symptoms.

One possible theoretical implication of the moderated mediation by youth sex is that theories of psychopathology would need to incorporate patterns of moderated mediation (Howe et al., 2002). This occurs when a set of maintenance factors function in one way for one group of youth and in a different way for another group of youth. If those factors are targeted in the approach program, the program will then have different effects on psychopathology in the two groups. It therefore follows that the relation between social anxiety and depressive symptoms may present itself differently for girls versus boys. Indeed, although changes in social anxiety and depressive symptoms were 
found for both boys and girls, changes in social anxiety symptoms mediated reductions in depressive symptoms and vice versa only for girls. This is an interesting finding because research shows that rates of social and depressive anxiety symptoms are more common in girls than boys (e.g., Aune \& Stiles, 2009; La Greca, 1999). This is an interesting finding also because it suggests that for boys, variables other than social anxiety mediate changes in depressive symptoms. This possibility indicates that there may be multiple and potentially different pathways that link social anxiety and depressive symptoms for girls versus boys (e.g., Cicchetti \& Rogosch 1996; Cicchetti \& Toth, 2009; Epkins \& Heckler, 2011).

Knowledge about treatment moderation and secondary outcomes that emerged in the present study could also inform classification efforts. In terms of treatment moderation, each comorbidity type - with depressive and externalizing disorders yielded a different pattern of treatment moderation. This suggests that the hypothesized moderators - depressive and externalizing disorders -- can be tentatively considered to represent distinct psychopathology from each other and from anxiety. In terms of secondary outcomes, when examining the relations between changes in anxiety and secondary depressive symptoms different patterns of moderated mediation emerged when we distinguished between general and social anxiety symptoms. This suggests that general and social anxiety can also be tentatively considered to represent distinct psychopathology. This distinction is important because some investigators suggest that at least two types of anxiety can be found in youth (i.e., separation anxiety, fears, obsessions and compulsions versus general anxiety and perhaps social anxiety) (e.g., Lahey et al., 2004). 
Furthermore, although changes in anxiety mediated changes in depressive symptoms and externalizing problem behaviors, the path coefficients revealed less than perfect relations and explained variances. The proportion of explained variance for changes in depressive symptoms was $44 \%$ and $31 \%$ when examined in relation to general and social anxiety symptoms, respectively. These less than perfect explained variances suggest that general and social anxiety symptoms are related yet not necessarily part of the same dimension as depressive symptoms. The proportion of explained variance for changes in externalizing problem behaviors in relation to general anxiety symptoms was $56 \%$. These less than perfect explained variances suggest that general anxiety symptoms and externalizing problem behaviors can also be tentatively considered to represent related but distinct psychopathology.

The suggestions regarding general and social anxiety symptoms, depressive symptoms, and externalizing problem behaviors as related but distinct psychopathology in the present study are tentative. This is because measures of anxiety symptoms may be linked to measures of depressive symptoms and externalizing problem behaviors for a number of reasons (Angold et al., 1999; Brady \& Kendall, 1992). One set of possibilities is methodological, stemming from overlapping constructs or items that may produce spurious relations. This methodological possibility is unlikely in the present study because each comorbidity type - with depressive and externalizing disorders - yielded a different pattern of treatment moderation and there were less than perfect path coefficients linking changes in anxiety to changes in secondary problems. The different pattern of treatment moderation by comorbidity type coupled with less than perfect path coefficients linking changes in anxiety to changes in secondary problems suggests that 
anxiety, depression, and externalizing problems can be tentatively considered to represent distinct yet related psychopathology with at least some nonoverlapping items.

Nevertheless, psychopathology researchers have paid far less attention to the development of strong measures of emotional distress (i.e., anxiety and depression) and externalizing problem behaviors than the development of measures of psychopathology with nonoverlapping items (e.g., Carter, Silverman, Allen, \& Ham, 2008; Stark \& Laurent, 2001). Research using measures of psychopathology with nonoverlapping items is needed to understand the relations between anxiety, depressive, and externalizing problems in ways that further informs classification efforts.

In addition, there are at least two major substantive reasons why anxiety symptoms may be linked to depressive symptoms and externalizing problem behaviors. The first substantive reason is that anxiety symptoms may be linked to depressive symptoms and externalizing problem behaviors simultaneously or in various combinations over time, producing phenomena described in developmental psychopathology as amplification, snowballing, transactional, cascade, or progressive effects (Cicchetti \& Tucker, 1994; Dodge \& Pettit, 2003; Hinshaw \& Anderson, 1996; Masten \& Coatsworth, 1998; Masten et al., 2005; Patterson, Reid, \& Dishion, 1992; Sameroff, 2000). Such progressions would account for what appear to be spreading or diffusing effects from anxiety to depressive symptoms and from anxiety symptoms to externalizing problem behaviors as those observed in the present study.

Although the first possibility emphasizes a causal relation of anxiety to depressive symptoms and of anxiety symptoms to externalizing problem behaviors, it is important to point out a second major substantive reason. Anxiety and depressive symptoms, on the 
one hand, and anxiety symptoms and externalizing problem behaviors, on the other hand, could become linked when a third variables contributes to both anxiety and secondary problems, "creating a spurious effect, the illusion of a causal link in either direction that is actually related to unmeasured variables" (p. 734, Masten et al., 2005). For example, the risk literature for social anxiety and depressive symptoms provides potential common causes that could underlie relations among these symptoms, including social skills deficits, loneliness, and having few or no friends as three relevant risk markers (e.g., Epkins \& Heckler, 2011). Similarly, the risk literature for anxiety and externalizing behaviors provides potential common causes that could underlie relations among these problems, including low socioeconomic status, low quality of parenting and parent-youth relationships (e.g., Masten et al., 2005). Future research is needed to explore these possibilities.

Empirical Contributions. Past psychotherapy research portrayed treatment moderation and secondary outcomes as related yet disparate issues. The present study contributes to the empirical literature through the integration of treatment moderation and secondary outcomes research into one investigation. The integration of these two lines of psychotherapy research in the present study is important because modifiable youth characteristics (e.g., comorbidity) can be examined not only as treatment moderators but also as secondary outcomes. The findings support the usefulness of this integrative approach because comorbidity had a moderating impact on treatment outcome and treatment had a modifiable impact on comorbidity. This integration therefore allows for understanding the impact of comorbidity on treatment outcome and alternate or complementary processes that occur in the extant approaches. The findings underscore 
the importance of addressing the effects of comorbidity on treatment outcome and the effects of treatment on comorbidity.

In terms of treatment moderation, the relatively large sample size compared to past research offered the opportunity to test interactions between youth characteristics and treatment approach thereby exploring the possibility that youth who share certain demographic and clinical characteristics may benefit more from CBT/P than GCBT, whereas youth who share other characteristics may benefit more from GCBT than $\mathrm{CBT} / \mathrm{P}$. Evaluation of youth age as a moderator of treatment outcome in the present study is important because researchers have long emphasized the need to consider the impact of developmentally relevant variables on treatment outcomes (e.g., Holmbeck et al., 2003; Kazdin, 2003).

The present study contributes to research also through its evaluation of comorbidity as a moderator of treatment outcome. In particular, the present study contributes to research through its evaluation of comorbidity -- with depressive and externalizing disorders - as a moderator of treatment outcome. The distinction of comorbidity with depressive and externalizing disorders in the present study is an important one because the patterns of differential treatment outcome varied by comorbidity type. CBT/P relative to GCBT lowered anxiety symptoms less for youth with comorbid depressive disorders than youth without comorbid depressive disorders. GCBT relative to CBT/P lowered anxiety symptoms less for youth with comorbid externalizing disorders than youth without comorbid externalizing disorders. The findings underscore the importance of potentially adapting the respective approaches to address the specific needs of youth with comorbid depressive and externalizing disorders. 
The present study makes an empirical contribution also through its evaluation of treatment moderation when the comparative treatment approaches are $\mathrm{CBT} / \mathrm{P}$ and GCBT. Research indicates that, on average, $\mathrm{CBT} / \mathrm{P}$ and GCBT do not differ in their relative efficacy when it comes to anxiety symptom reduction (Marin, 2010). Yet these outcomes are patterns that are seen on average when youth who have undergone treatment are studied as a group. The present study indicates that older youth and youth with comorbid depressive disorders compared to younger youth and youth without comorbid depressive disorders benefit more from GCBT than CBT/P according to parent report at posttreatment. Youth with comorbid externalizing disorders compared to youth without comorbid externalizing disorders benefit less from GCBT than CBT/P according to parent report at posttreatment. These patterns of differential treatment outcome are important because youth with anxiety disorders are heterogeneous in nature and often differ in age and comorbidities (e.g., Kendall et al., 2010). Understanding how best to treat anxious youth requires knowledge about factors and processes that lead to differential treatment outcome (e.g., Kraemer et al., 2002).

It is important to note that the present study is the first in the youth anxiety literature to test for treatment moderators using single degree of freedom interaction contrasts as recommended by Jaccard and Guilamo-Ramos (2002). None of the past studies on treatment moderation reported single degree of freedom interaction parameters. Instead, past studies used the traditional approach to test for moderators. The traditional approach to test for moderators involves visual inspection of means and analyses of simple main effects in factorial designs (Jaccard \& Guilamo-Ramos, 2002). The traditional approach to test for moderators is a limitation of past research because the 
visual inspection of means and analyses of simple main effects do not formerly and effectively address interaction effects (Jaccard \& Guilamo-Ramos, 2002).

The present study moved beyond efficacy research to examine anxiety symptom reduction as the mechanism responsible for changes in secondary depressive symptoms and externalizing problem behaviors (e.g., La Greca et al., 2009). Previous research found that anxious youth have difficulty managing worry, sadness, and anger due to their experiencing emotions with high intensity (Suveg \& Zeman, 2004). Consistent with this research, clinical observations show that when parents ask their child to face his or her fears, a natural response on the child's part is to either withdrawn or externalize in protest. The findings suggest that once youth learn to apply cognitive skills and behavioral strategies to face their fears as part of the treatment approaches, their anxiety lessens and they experience a concomitant decrease in depressive and externalizing problems. Reductions in anxiety symptoms in other words mediate reductions in secondary, non-targeted, depressive symptoms and externalizing problem behaviors.

The present study moved beyond efficacy research to examine directionality of change in the relations between changes in primary anxiety and secondary outcomes. Importantly, the directionality of change was examined in the relations between different types of anxiety (e.g., general versus social anxiety symptoms) and secondary problems (i.e., depressive symptoms, externalizing problem behaviors). The findings support the usefulness of this approach because the directionality of change presented differently when depressive symptoms were examined in relation to social versus general anxiety symptoms. 
Clinical Implications. The results of this study have several clinical implications.

First, the results suggest that developmental considerations are important when assigning youth to effective approaches. That CBT/P relative to GCBT lowered anxiety symptoms more for younger children than older children suggests that the inclusion of training in parenting skills and parent-youth relationships may be an important developmental consideration when matching youth to effective approaches. That GCBT relative to $\mathrm{CBT} / \mathrm{P}$ lowered anxiety symptoms more for older children than younger children suggests that the inclusion of training in social skills and peer-youth relationships may also be an important developmental consideration when matching youth to effective approaches.

Second, the results suggest that it is important for clinicians to assess for comorbid depressive and externalizing disorders in youth presenting for anxiety treatment in order to inform personalized decision making in treatments (NIMH; U.S. Department of Health and Human Services, 2008). Ascertaining that youth have both anxiety and depressive disorders may lead to a decision to implement a treatment approach that involves targeting of social skills and peer-youth relationships, such as GCBT. The main therapeutic components of such treatment approaches may involve training children in being more helpful and positive toward other children in the group in the context of anxiety reduction, having children accept the help and support of other group members in role-plays, and training children in social skills (e.g., compliment giving, conversational skills).

Ascertaining that youth have both anxiety and externalizing disorders may lead to a decision to implement a treatment approach that involves targeting of parenting skills, 
such as $\mathrm{CBT} / \mathrm{P}$. The main therapeutic components of such treatment approaches may involve training parents in managing their children's anxiety and avoidant behaviors and having parents set appropriate contingencies for successful child non-avoidance. Implementation of CBT/P may facilitate the transfer of control over the skills for improving anxiety even in the presence of externalizing disorders (Silverman \& Kurtines, 1996). Ascertaining that anxious youth do not have comorbid depressive and externalizing disorders may lead to a decision to implement either CBT/P or GCBT with the expectation that anxiety symptom reduction would be of similar strength irrespective of choice between treatment approaches. Choice between treatment approaches for anxious youth without comorbid depressive and externalizing disorders could be based on pragmatic considerations such as the availability of $\mathrm{CBT} / \mathrm{P}$ and GCBT and referral rates (e.g., Liber et al., 2008).

Third, reductions in depressive symptoms and externalizing problem behaviors in this dissertation study are highly encouraging especially since they were not directly targeted. Such reductions support the generalizability of positive treatment effects from the targeted, anxiety symptoms to secondary, non-targeted, depressive and externalizing problem behaviors through a process of mediation. By elucidating anxiety symptom reduction as the mechanism of change, clinicians can focus on anxiety symptom reduction not only as a mean in itself but also an opportunity to reduce and stop the progression of depressive symptoms and externalizing problem behaviors. Results of the present study underscore that secondary outcome measures demonstrate sensitivity to treatment and their inclusion in RCTs has practical utility. Given the limited funds often available for approach efforts coupled with the time resources necessary to attend 
multiple treatment programs, targeting anxious youth with CBT approaches is an effective, cost- and time-efficient alternative to altering not only anxiety but also depressive symptoms and externalizing problem behaviors that co-occur with it.

\section{Limitations and Directions for Future Research}

Limitations of the present study should be noted. First, although the present study drew on the largest RCT to date for youth anxiety and its disorders, this RCT was designed to investigate a main effect for treatment and not designed to investigate interaction effects. Adequate tests of interaction effects may require an even larger sample size than for capturing main effects. One limitation is the relatively small number of youth with comorbid depressive and externalizing disorders in each of the treatments. The fact that there was evidence of treatment moderation based on comorbidity with depressive and externalizing disorders suggests that the presence of comorbidity is an important moderator of treatment outcome in CBTs involving parents and peers.

The sample size considerations when coupled with the above significant findings raise the need for an adequately powered treatment study testing whether comorbidity -with depressive and externalizing disorders -- interacts with treatment approaches to either strengthen or weaken outcome. Such a treatment study would benefit from inclusion of a large number of youth with comorbid depressive and externalizing disorders in each of the approaches, as well as a wide range of outcome variables tailored to the hypothesized advantages of $\mathrm{CBT} / \mathrm{P}$ and GCBT for youth with various comorbidity profiles. These advantages include an increase in parenting skills in CBT/P for youth with comorbid externalizing disorders and an increase in social skills in GCBT for youth with comorbid depressive disorders. Future research is also encouraged to determine what 
modifications in the extant treatment approaches may be needed for youth with various comorbidity profiles.

Second, although developmental psychopathology theory and research findings have been invoked in the generation of hypotheses and the explanation of findings, the present study was not designed as a direct test of developmental psychopathology. Knowledge of moderators that emerged in the present study, nevertheless, helps set the stage for future research hypotheses and investigations that could shed light into the reasons why the moderators operated the way they did in the present study (Kraemer et al., 2002).

Methodologically, the findings obtained in the present study emphasize the importance of assessing multiple domains of psychopathology from baseline and then repeatedly through the duration of treatment and follow-up periods in future research. Multiple assessments spaced relatively close to each other will allow capturing developmental cascades across various domains of psychopathology over time. The possibility of developmental cascades underscores the importance of conducting future research into "the processes, timing, and conditions of spreading and amplifying effects" and of learning "when to do what to interrupt negative progressions" and foster positive change (p. 742, Masten et al., 2005). Future research is therefore encouraged to take a systematic approach to measurement in order to fully capture developmental cascades. If developmental cascades do exists, as the present study began to show, it is possible that the best way to stop the progression of one type of problem (e.g., depression) is to intervene in another (e.g., anxiety) (Masten et al., 2005). Additional knowledge of what, 
when, how, and for whom cascades occur will help strategize treatments so they are effective in reducing multiple emotional and behavioral problems. 


\section{LIST OF REFERENCES}

Achenbach, T. M. (1991). Manual for the Child Behavior Checklist and Revised Child Behavior Profile (Revised). Department of Psychiatry, University of Vermont, Burlington.

Achenbach, T. M. (1997). Manual for the Young Adult Self-Report and Young Adult Behavior Checklist. Burlington, VT: University of Vermont Department of Psychiatry.

Achenbach, T. M., \& Edelbrock, C. (1983). Manual for the Child Behavior Checklist and the Revised Child Behavior Profile. Burlington, VT: University of Vermont Department of Psychiatry.

Achenbach, T. M., McConaughy, S. H., \& Howell, C. T. (1987). Child-adolescent behavioral and emotional problems: Implications of cross-informant correlations for situational specificity. Psychological Bulletin, 101, 213-232.

Aiken, L. S., West, S. G. (1991). Multiple regression: Examining and interpreting Interactions. Newbury Park, CA: Sage.

Alfano, C. A., Pina, A. A., Villalta, I. K., Beidel, D. C., Ammerman, R. T, \& Crosby, L. E. (2009). Mediators and moderators of outcome in the behavioral treatment of childhood social phobia. Journal of the American Academy of Child and Adolescent Psychiatry, 48, 945-953.

Aune, T., \& Stiles, T. C. (2009). The effects of depression and stressful life events on the development and maintenance of syndromal social anxiety: Sex and age differences. Journal of Clinical Child and Adolescent Psychology, 38, 501-512.

Angold, E., Costello, J., \& Erkanli, A. (1999). Comorbidity. Journal of Child Psychology and Psychiatry, 40, 57-87.

Barrett, P. M. (1998). Evaluation of cognitive-behavioral group treatments for childhood anxiety disorders. Journal of Clinical Child Psychology, 27, 459-468.

Barrett, P. M., Dadds, M. R., \& Rapee, R. M. (1996). Family treatment of childhood anxiety: A controlled trial. Journal of Consulting and Clinical Psychology, 64, 333-342.

Barrett, P., Duffy, A., Dadds, M. \& Rapee, R. (2001). Cognitive-behavioral treatment of anxiety disorders in children: Long-term (6-year) follow-up. Journal of Consulting and Clinical Psychology, 69, 135-141. 
Beidel, D. C., Turner, S. M., \& Morris, T. L. (1995). A new inventory to assess childhood social anxiety and phobia: the Social Phobia and Anxiety Inventory for Children. Psychological Assessment, 7, 73-79.

Beidel, D. C., Turner, S. M., \& Morris, T. L. (1998). Social Effectiveness Therapy for Children: A treatment manual. Unpublished manuscript, Medical University of South Carolina.

Beidel, D. C., Turner, S. M., \& Morris, T. L. (1999). Psychopathology of childhood social phobia. Journal of American Academy of Child and Adolescent Psychiatry, $38,643-650$.

Beidel, D. C., Turner, S. M., \& Morris, T. L. (2000). Behavioral treatment of childhood social phobia. Journal of Consulting and Clinical Psychology, 68, 1072-1080.

Beidel, D. C., Turner, S. M., Sallee, F. R., Ammerman, R. T., Crosby, L. A., \& Pachak, S. (2007). SET-C versus fluoxetine in the treatment of childhood social phobia. Journal of the American Academy of Child and Adolescent Psychiatry, 46, 16221632.

Berman, S. L., Weems, C. F., Silverman, W. K., \& Kurtines, W. M. (2000). Predictors of outcome in exposure-based cognitive and behavioral treatments for phobic and anxiety disorders in children. Behavior Therapy, 31, 713-731.

Bird, H. R., Gould, M. S., \& Staghezza, B. (1992). Aggregating data from multiple informants in child psychiatry epidemiological research. Journal of the American Academy of Child and Adolescent Psychiatry, 31, 78-85.

Birmaher, B., Khetarpal, S., Brent, D., Cully, M., Balach, L., Kaufman, J., et al. (1997). The Screen for Child Anxiety Related Emotional Disorders (SCARED): Scale construction and psychometric properties. Journal of the American Academy of Child and Adolescent Psychiatry, 36, 545-553.

Bodden, D. H. M., Bögels, S. M., Nauta, M. H., de Hann, E. Ringrose, J., Abbelboom, C., et al. (2008). Child versus family cognitive-behavioral therapy in clinically anxious youth: An efficacy and partial effectiveness study. Journal of the American Academy of Child and Adolescent Psychiatry, 47, 1384-1394.

Blair, R. C., Higgins, J. J., Topping, M. E., \& Mortimer, A. L. (1983). An investigation of the robustness of the $t$ test to unit of analysis violations. Educational and Psychological Measurement, 43, 69-80.

Brady, E. U., \& Kendall, P. C. (1992). Comorbidity of anxiety and depression in children and adolescents. Psychological Bulletin, 111, 244-255. 
Campbell, S. B., Shaw, D. S., \& Gilliom, M. (2000). Early externalizing behavior problems: Toddlers and preschoolers at risk for later maladjustment. Development and Psychopathology, 12, 467-488.

Carter, R., Silverman, W. K., Allen, A., \& Ham, L. (2008). Measures matter: The relative contribution of anxiety and depression to suicidal ideation in clinically referred anxious youth using brief versus full length questionnaires. Depression and Anxiety, 25, 27-35.

Chambless, D. L., \& Hollon, S. D. (1998). Defining empirically supported therapies. Journal of Consulting and Clinical Psychology, 66, 7-18.

Cicchetti, D., \& Schneider-Rosen, K. (1986). An organizational approach to childhood depression. In M. Rutter, C. Izard \& P. Read (Eds.), Depression in young people, clinical and developmental perspectives (pp. 71-134). New York: Guilford.

Cicchetti, D., \& Toth, S. L. (1992). The role of developmental theory in prevention and intervention. Development and Psychopathology, 4, 489-493.

Cicchetti, D., \& Toth, S. L. (1998). The development of depression in children and adolescent. American Psychologist, 53, 221-241.

Crawley, S., Beidas, R.S., Benjamin, C., Martin, E., \& Kendall P.C. (2008). Treating socially phobic youth with CBT: Differential outcomes and treatment considerations. Behavioral and Cognitive Psychotherapy, 36, 379-389.

Costello, E. J., Egger, H. L., \& Angold, A. (2005). The developmental epidemiology of anxietydisorders: Phenomenology, prevalence, and comorbidity. Child and Adolescent Psychiatric Clinics of North America, 14, 631-648.

Costello, E. J., \& Angold, A. (1995). Developmental epidemiology. In D. Cicchetti \& D. Cohen (Eds.), Developmental psychopathology (Vol. 1, pp. 23-56). New York: Wiley.

Costello, E. J., Egger, H. L., Copeland, W., Erkanli, A., \& Angold, A. (2011). The developmental epidemiology of anxiety disorders: phenomenology, prevalence, and comorbidity. In W. K. Silverman \& A. Field (Eds.), Anxiety disorders in children and adolescents: Research, assessment and intervention (pp. 56-75). Cambridge, U. K.: Cambridge University Press.

DiNardo, P. A., Brown, T. A., \& Barlow, D. H. (1994). Anxiety Disorders Interview Schedule for DSM-IV: Lifetime Version (ADIS-IV-L). Albany, NY: Graywind Publications. 
Epkins, C. C. \& Heckler, D. D. (2011). Integrating etiological models of social anxiety and depression in youth: Evidence for a cumulative interpersonal risk model highlighting parent and peer relationships and implications for understanding comorbidity. Clinical Child and Family Psychology Review, 14, 329-376.

Gallagher, H. M., Rabian, A. A., \& McCloskey, M. S. (2004). A brief group cognitivebehavioral intervention for social phobia in childhood. Journal of Anxiety Disorders, 18, 459-479.

Gallerani, C. M., Garber, J., \& Martin, N. C. (2010). The temporal relation between depression and comorbid psychopathology in adolescents at varied risk for depression. Journal of Child Psychology and Psychiatry, 50, 242-249.

Ginsburg G. S., Kendall, P. C., Sakolsky, D., Compton, S. N., Piacentini, J., Albano, A. M., et al. (2011). Remission after acute treatment in children and adolescents with anxiety disorders: Findings from the CAMS. Journal of Consulting and Clinical Psychology, 79, 806-813.

Ginsburg, G., La Greca, A. M., \& Silverman, W. K. (1998). Social anxiety in children with anxiety disorders: Relations with social and emotional functioning. Journal of Abnormal Child Psychology, 26, 175-185.

Ginsburg, G. S., Silverman, W. K., \& Kurtines, W. M. (1995). Family involvement in treating children with phobic and anxiety disorders: A look ahead. Clinical Psychology Review, 15, 457-473.

Goodwin, R. D., Fergusson, D. M., \& Horwood, L. J. (2004). Early anxious/withdrawn behaviours predict later internalizing disorders. Journal of Child Psychology and Psychiatry, 45, 874-883.

Hammen, C., Shih, J. H., \& Brennan, P.A (2004). Intergenerational transmission of depression: Test of an interpersonal stress model in a community sample. Journal of Consulting and Clinical Psychology, 72, 511-522.

Hayward, C., Varardy, S., Albano, A. M., Thienemann, M., Henderson, L., \& Schatzberg, A. F. (2000). Cognitive-behavioral group therapy for social phobiain female adolescents: Results of a pilot study. Journal of the American Academy of Child and Adolescent Psychiatry, 39, 721-726.

Hinshaw, S. P. (1987). On the distinction between attentional deficits/hyperactivity and conduct problems/aggression in child psychopathology. Psychological Bulletin, 101, 443-463. 
Hinshaw, S. P. (2007). Moderators and mediators of treatment outcome for youth with ADHD: Understanding for whom and how interventions work. Journal of Pediatric Psychology, 32, 664-675.

Huber, P. J. (1967). The behavior of maximum likelihood estimates under nonstandard intervention. Proceedings of the Fifth Berkeley Symposium on Mathematical Statistics and Probability (pp. 221-233). Berkeley, CA: University of California Press.

Jaccard, J. (1998). Interaction effects in factorial analysis of variance. Newbury Park, CA: Sage.

Jaccard, J., \& Guilamo-Ramos, V. (2002). Analysis of Variance Frameworks in Clinical Child and Adolescent Psychology: Issues and Recommendations. Journal of Clinical Child \& Adolescent Psychology, 31, 130-146.

Jaccard, J., \& Turrisi, R. (2003). Interaction effects in multiple regression (2nd ed.). Thousand Oaks, CA: Sage.

Jaccard, J., Turrisi, R., \& Wan, C. (1990) Interaction effects in multiple regression. Newbury Park: Sage.

Jaccard, J., \& Wan, C. (1996). LISREL analyses of interaction effects in multiple regression. Newbury Park, CA: Sage.

Joiner, T. E., Coyne, J. C., Blalock, J. (1999). On the interpersonal nature of depression: Overview and synthesis. In T. E. Joiner \& J. C. Coyne (Eds.), The interactional nature of depression. (pp. 3- 19). Washington, D.C.: American Psychological Association.

Kazdin, A. E. (1994). Methodology, design, and evaluation in psychotherapy research. In A. E. Bergin \& S. L. Garfield (Eds.), Handbook of psychotherapy and behavior change (4th ed.) (pp.19-71). New York: Wiley.

Kazdin, A. E. (2001). Progression of therapy research and clinical application of treatment require better understanding of the change process. Clinical Psychology: Science and Practice, 8, 143-151.

Kazdin, A. E. (2007). Mediators and mechanisms of change in psychotherapy research. Annual Review of Clinical Psychology, 3, 1-27.

Kazdin, A. E. (2008). Evidence-based treatment and practice: New opportunities to bridge clinical research and practice, enhance the knowledge base, and improve patient care. American Psychologist, 63, 146-159. 
Kazdin, A. E., Nock, M. K. (2003). Delineating mechanisms of change in child and adolescent therapy: Methodological issues and research recommendations, Journal of Child Psychology and Psychiatry 44, 1116-1129.

Kendall, P. C. (1994). Treating anxiety disorders in children: Results of a randomized clinical trial. Journal of Consulting and Clinical Psychology, 62, 200-210.

Kendall, P. C., Compton, S. N., Walkup, J. T., Birmaher, B., Albano, A. M., Sherrill, J., et al. (2010). Clinical characteristics of anxiety disordered youth. Journal of Anxiety Disorders, 24, 360-365.

Kendall, P. C., Flannery-Schroeder, E., C., Panichelli-Mindel, S. M., Southam-Gerow, M., Henin, A., \& Warman, M. (1997). Therapy for youth with anxiety disorders: A second randomized clinical trial. Journal of Consulting and Clinical Psychology, 65, 366-380.

Kendall, P. C., Hudson, J., Gosch, E., Flannery-Schroeder, E., \& Suveg, C. (2008). Child and family therapy for anxiety-disordered youth: results of a randomized clinical trial. Journal of Consulting and Clinical Psychology, 76, 282-297.

Kovacs, M. (1992). Children's Depression Inventory Manual. New York: Multi-Health Systems.

Kraemer, H. C., Wilson, T., Fairburn, C. G., \& Agras, S. (2002). Mediators and moderators of treatment effects in randomized clinical trials. Archives of General Psychiatry, 59, 877-883.

La Greca, A. M. (1999). Social anxiety scales for children and adolescents: Manual and instructions for the SASC, SASC-R, SAS-A (adolescents), and parent versions of the scales. Miami, FL: University of Miami, Department of Psychology.

La Greca, A. M., Silverman, W. K., \& Lochman, J. E. (2009). Moving beyond efficacy and effectiveness in child and adolescent intervention research. Journal of Consulting and Clinical Psychology, 77, 3, 373-382.

La Greca, A. M., \& Stone, W. L. (1993). Social anxiety scale for children-Revised: Factor structure and concurrent validity. Journal of Clinical Child Psychology, $22,17-27$.

Lahey, B. B., Applegate, B., Waldman, I. D., Loft, J. D., Hankin, B. L., Rick, J. (2004). The structure of child and adolescent psychopathology: Generating new hypotheses. Journal of Abnormal Psychology, 113, 358-385. 
Last, C. G., Perrin, S., Hersen, M., \& Kazdin, A. E. (1996). A prospective study of childhood anxiety disorders. Journal of the American Academy of Child and Adolescent Psychiatry, 35, 1502-1510.

Lewinsohn, P. M. (1974). A behavioral approach to depression. In R. M. Friedman, \& M. M. Katz (Eds.), The psychology of depression: contemporary theory and research. New York: Wiley

Liber, J. M., Van Widenfelt, B. M., Utens, E. M. W. J., Ferdinand, R. F., Van der Leeden, A. J. M., \& Van Gastel, W. (2008). No differences between group versus individual treatment of childhood anxiety disorders in a randomized clinical trial. Journal of Child Psychology and Psychiatry, 49, 886-893.

LoCastro, J. S.,Youngblood, M., Cisler, R. A., Mattson, M. E., Zweben, A., Anton, R. F. et al., (2009). Alcohol Treatment Effects on Secondary Nondrinking Outcomes and Quality of Life: The COMBINE Study. Journal of Studies on Alcohol and Drugs, 70, 186-196.

Lyneham, H. J. \& Rapee, R. M. (2011). Prevention of child and adolescent anxiety disorders. In Silverman, W. K. \& Field, A. (Eds). Anxiety disorders in children and adolescents: Research, assessment, and intervention, pp. 349-365. Cambridge, U. K.: Cambridge University Press.

MacKinnon, D. P., Lockwood, C. M., Hoffman, J. M., West, S. G., \& Sheets, V. (2002). A comparison of methods to test mediation and other intervening variable effects. Psychological Methods, 7, 83-104.

Manassis, K., Mendlowitz, S. L., Scapillato, D., Avery, D., Fiksenbaum, L., \& Freire, M. (2002). Group and individual cognitive-behavioral therapy for childhood anxiety disorders: A randomized trial. Journal of the American Academy of Child and Adolescent Psychiatry, 41, 1423-1430.

March, J. S., Parker, J., Sullivan, K., Stallings, P., \& Conners, K. (1997). The Multidimensional Anxiety Scale for Children (MASC): Factor structure, reliability and validity. Journal of the American Academy of Child and Adolescent Psychiatry, 36, 554-565.

Marin, C. E. (2010). Parental involvement and group cognitive behavioral treatment for anxiety disorders in children and adolescents: Treatment specificity and mediation effects of parent and peer variables (Doctoral dissertation, Florida International University). Retrieved from http://digitalcommons.fiu.edu/etd/256. 
Masten, A. S., Roisman, G. I., Long, J. D., Burt, K. B., Obradović, J., Riley, J. R., et al. (2005). Developmental cascades: Linking academic achievement, externalizing and internalizing symptoms over 20 years. Developmental Psychology, 41, 733746.

Mathew, A. R., Pettit, J. W., Lewinsohn, P.M., Seeley, J. R., \& Roberts, R. E. (2011). Comorbidity between major depressive disorder and anxiety disorders: Shared etiology or direct causation? Psychological Medicine. Advance online publication.

Mendolowitz, S.L., Manassis, K., Bradley, S., Scapillato, D., Miezitis, S., Shaw, B.F. (1999). Cognitive-behavioral group treatments in childhood anxiety disorders: the role of parental involvement. Journal of the American Academy of Child and Adolescent Psychiatry, 38, 1223-1229.

Motoca, L. M. (2009). Predictors of treatment outcome in cognitive behavioral therapy For anxious youth (Master's thesis, Florida International University).

Motoca, L. M., Williams, S., \& Silverman, W. K. (2012). Social Skills as a mediator between anxiety symptoms and peer interactions among children and adolescents. Journal of Clinical Child and Adolescent Psychology, 41, 329-336.

Moscovitch, D. A., Hofmann, S.G., Suvak, M. K., In-Albon T. (2005). Mediation of changes in anxiety and depression during treatment of social phobia. Journal of Consulting and Clinical Psychology, 73, 945-952.

Muthén, L. K., \& Muthén, B. O. (2007). Mplus User's Guide (Sixth Edition). Los Angeles, CA: Muthén \& Muthén.

National Institute of Mental Health. (2008). The National Institute of Mental Health strategic plan (NIH Publication No. 08-6368). Retrieved from http://www.nimh.nih.gov/about/strategic-planning-reports/index.shtml.

Nauta, M., Scholing, A., Emmelkamp, P. M. G., \& Minderaa, R. (2003). Cognitive behavioral therapy for children with anxiety disorders: No additional effect of parent training. Journal of the American Academy of Child \& Adolescent Psychiatry, 42, 1270-1278.

Ollendick, T. H., Jarrett, M. A., Grills-Taquechel, A. E., Hovey, L. D., \& Wolff, J. (2008). Comorbidity as a predictor and moderator of treatment outcome in youth with anxiety, affective, $\mathrm{AD} / \mathrm{HD}$, and oppositional/conduct disorders. Clinical Psychology Review, 28, 1447-1471. 
Ollendick, T. H., Öst, L.-G., Reuterskiöld, L., \& Costa, N. (2010). Comorbidity in youth with specific phobias: Impact of comorbidity on treatment outcome and the impact of treatment on comorbid disorders. Behaviour Research and Therapy, 48, 827-831.

Parker, J., Rubin, K. H., Erath, S., Wojslawowicz, J. C., \& Buskirk, A. A. (2006). Peer relationships and developmental psychopathology. In D. Cicchetti \& D. Cohen (Eds.), Developmental Psychopathology: Risk, Disorder, and Adaptation (2nd edition), Vol. 2. (pp. 419-493). New York: Wiley.

Pina, A. A., Silverman, W. K., Fuentes, R. M., Kurtines, W. M., \& Weems, C. F. (2003). Exposure-based cognitive-behavioral treatment for phobic and anxiety disorders: Treatment effects and maintenance for Hispanic-Latino relative to European American youth. Journal of the American Academy of Child and Adolescent Psychiatry, 42, 1179-1187.

Pine, D. S., Cohen, P., Gurley, D., Brook, J., \& Ma, Y. (1998). The risk for early adulthood anxiety and depressive disorders in adolescents with anxiety and depressive disorders. Archives of General Psychiatry, 55, 56-64.

Reynolds, C. R., \& Richmond, B. O. (1978). What I think and feel: A revised measure of children's manifest anxiety. Journal of Abnormal Child Psychology, 6, 271-280.

Saavedra, L., Silverman, W. K., Morgan-Lopez, A. A., \& Kurtines, W. M. (2010). Cognitive behavioral treatment for childhood anxiety disorders: Long-term effects on anxiety and secondary disorders in young adulthood. Journal of Child Psychology and Psychiatry, 51, 924-934.

Schniering, C.A., \& Rapee, R.M. (2002). Development and validation of a measure of children's automatic thoughts: The Children's Automatic Thoughts Scale. Behaviour Research and Therapy: Behavioral Assessment Section, 40, 10911109.

Sameroff, A. J. (2000). Developmental systems and psychopathology. Development and Psychopathology, 12, 297-312.

Silverman, W. K., \& Albano, A. M. (1996). Anxiety Disorders Interview Schedule for Children-IV (Child and Parent Versions). San Antonio, TX: Psychological Corporation.

Silverman, W. K., Kurtines, W. M., Ginsburg, G. S., Weems, C. F., Rabian, B., \& Serafini, L. T. (1999a). Contingency management, self-control, and education support in the treatment of childhood phobic disorders: A randomized clinical trial. Journal of Consulting and Clinical Psychology, 67, 675-687. 
Silverman, W. K., Kurtines, W. M., Ginsburg, G. S., Weems, C. F., Lumpkin, P. W., \& Hicks-Carmichael, D. (1999b). Treating anxiety disorders in children with group cognitive behavior therapy: A randomized clinical trial. Journal of Consulting and Clinical Psychology, 67, 995-1003.

Silverman, W. K., Kurtines, W. M., Jaccard, J., \& Pina, A. (2009). Directionality of change in youth anxiety treatment involving parents: An initial examination. Journal of Consulting and Clinical Psychology.

Silverman, W. K., \& Motoca, L. M. (2011). Treatment: Update and recommendations. In Silverman, W. K., \& Field, A. (Eds.). Anxiety disorders in children and adolescents: Research, assessment and intervention, pp. 392-418. Cambridge, U. K.: Cambridge University Press.

Silverman, W. K., Pina, A. A., \& Viswesvaran, C. (2008). Evidence-based psychosocial treatments for phobic and anxiety disorders in children and adolescents. Journal of Clinical Child and Adolescent Psychology, 37, 105-130.

Spence, S. H. (1998). A measure of anxiety among anxious children. Behaviour Research and Therapy, 36, 545-566.

Spence, S. H., Donovan, C. \& Brechman-Toussaint, M. (1999). Social skills, social outcomes and cognitive features of childhood social phobia. Journal of Abnormal Psychology, 108, 211-221

Spence, S. H., Donovan, C., \& Brechman-Toussaint, M. (2000). The treatment of childhood social phobia: The effectiveness of a social skills training-based, cognitive behavioural intervention, with and without parental involvement. Journal of Child Psychology and Psychiatry, 41, 713-726.

Spielberger, C.D. (1973). Manual for the State-Trait Anxiety Inventory for Children. Palo Alto, CA: Consulting Psychologists Press.

Stark, K. D., \& Laurent, J. (2001). Joint factor analysis of the Children's Depression Inventory and the Revised Children's Manifest Anxiety Scale. Journal of Clinical Child Psychology, 30, 552-567.

Strauss, C. C., Lahey, B. B., Frick, P., Frame, C. L., \& Hynd, G. (1988). Peer social status of children with anxiety disorders. Journal of Consulting and Clinical Psychology, 56, 137- 141.

Strauss, C. C., Lease, C. A., Kazdin, A. E., Dulcan, M. K., \& Last, C. G. (1989). Multimethod assessment of the social competence of children with anxiety disorders. Journal of Clinical Child Psychology, 18, 184-189. 
Strauss, C.C., Frame, C.L., \& Forehand, R.L. (1987). Psychosocial impairment associated with anxiety in children. Journal of Clinical Child Psychology, 16, 235-239.

Thelen, E. (1989). Self-organization in developmental processes: Can systems approaches work? In M. R. Gunnar \& E. Thelen (Eds.), Systems and development: The Minnesota Symposia on Child Psychology (Vol. 22, pp. 77117). Hillsdale, NJ: Erlbaum.

Turner, S. M., Beidel, D. C., \& Cooley-Quille, M. R. (1997). Social effectiveness therapy: A program for overcoming social anxiety and social phobia.

Toronto, Ontario, Canada: Multi-Health Systems.

U.S. Department of Commerce. (2002). Resident population estimates of the United States by sex, race and Hispanic origin. Retrieved March 15, 2012, from the World Wide Web: http://eire.census.gov/popest/archives/national/nation3/intfile31.txt.

Van Lier, P. A. C., \& Koot, H. M. (2010). Developmental cascades of peer relations and symptoms of externalizing and internalizing problems from kindergarten to fourth-grade elementary school. Development and Psychopathology, 22, 569-582

White, H. (1980). A heteroskedasticity-consistent covariance matrix estimator and a direct test for heteroskedasticity. Econometrica, 48, 817-830.

Wood, J. J., McLeod, B. D., Sigman, M., Hwang,W. C., \& Chu, B. C. (2003). Parenting and childhood anxiety: theory, empirical findings, and future directions. Journal of Child Psychology and Psychiatry, 44, 134-145.

Woodward, L.J. and Fergusson, D. M. (2001). Life course outcomes of young people with anxiety disorders in adolescence. Journal of the American Academy of Child and Adolescent Psychiatry, 40, 1086-1093.

Wood, J. J., Piacentini, J. C., Southam-Gerow, M., Chu, B.C., \& Sigman, M. (2006). Family cognitive behavioral therapy for child anxiety disorders. Journal of the American Academy of Child and Adolescent Psychiatry, 45, 314-321.

Wothke, W. (2000). Longitudinal and multi-group modeling with missing data. In T. D. Little, K. U. Schnabel, \& J. Baumert (Eds.) Modeling longitudinal and multiple group data: Practical issues, applied approaches and specific examples. Mahwah, NJ: Erlbaum. 
APPENDICES 
Table 1. Single Degree of Freedom and Interaction Contrasts: RCMAS

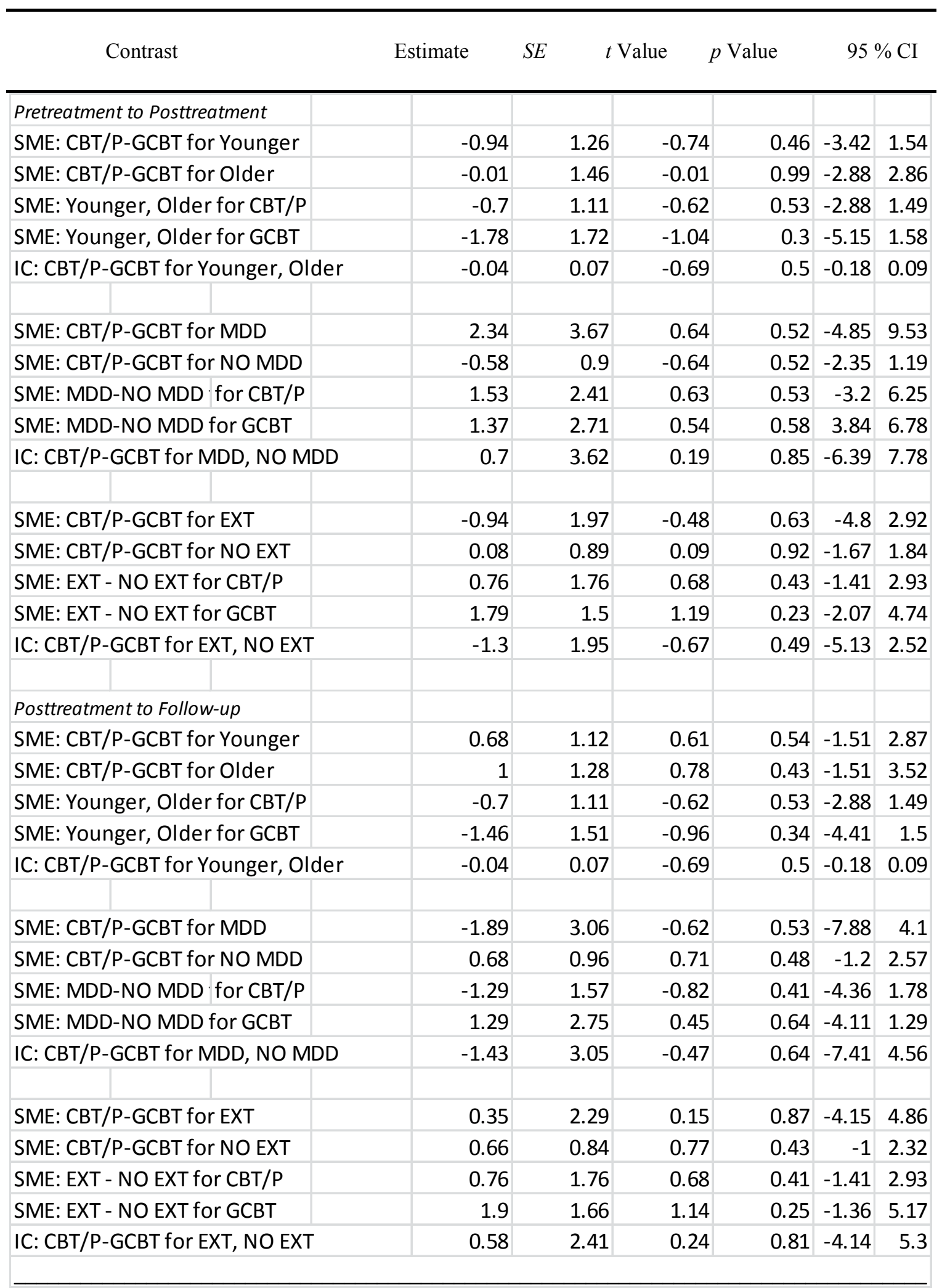


Table 2. Single Degree of Freedom and Interaction Contrasts: RCMAS/P

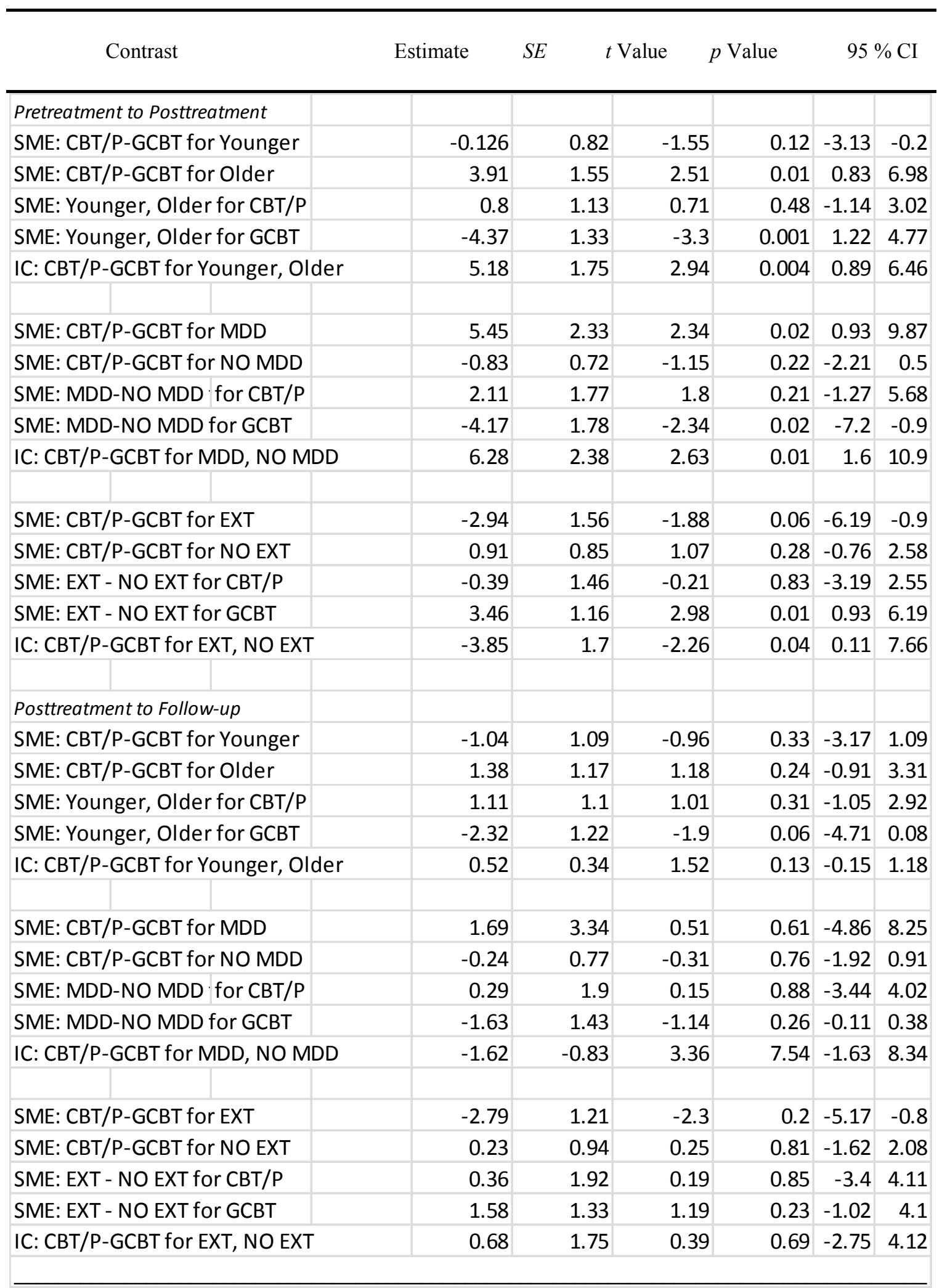


Table 3. Cell Mean Anxiety Residual Change Scores: Pre to Post

\begin{tabular}{|c|c|c|}
\hline Moderator & $\mathrm{CBT} / \mathrm{P}$ & GCBT \\
\hline \multicolumn{3}{|l|}{ RCMAS } \\
\hline \multicolumn{3}{|l|}{ Youth Age } \\
\hline Younger & 7.8 & 7.87 \\
\hline Older & 7.9 & 8.03 \\
\hline \multicolumn{3}{|c|}{ Depressive Disorders } \\
\hline MDD & 10.75 & 10.6 \\
\hline No MDD & 8.82 & 9.34 \\
\hline \multicolumn{3}{|c|}{ Externalizing Disorders } \\
\hline EXT & 5.05 & 6.38 \\
\hline No EXT & 4.12 & 4.45 \\
\hline \multicolumn{3}{|l|}{ RCMAS/P } \\
\hline \multicolumn{3}{|l|}{ Youth Age } \\
\hline Younger & 7.99 & 9.26 \\
\hline Older & 8.80 & 4.89 \\
\hline \multicolumn{3}{|c|}{ Depressive Disorders } \\
\hline MDD & 10.12 & 4.67 \\
\hline No MDD & 8.01 & 8.84 \\
\hline \multicolumn{3}{|c|}{ Externalizing Disorders } \\
\hline EXT & 7.87 & 10.82 \\
\hline No EXT & 8.26 & 7.35 \\
\hline
\end{tabular}


Table 4. Cell Mean Anxiety Residual Change Scores: Post to Follow-up

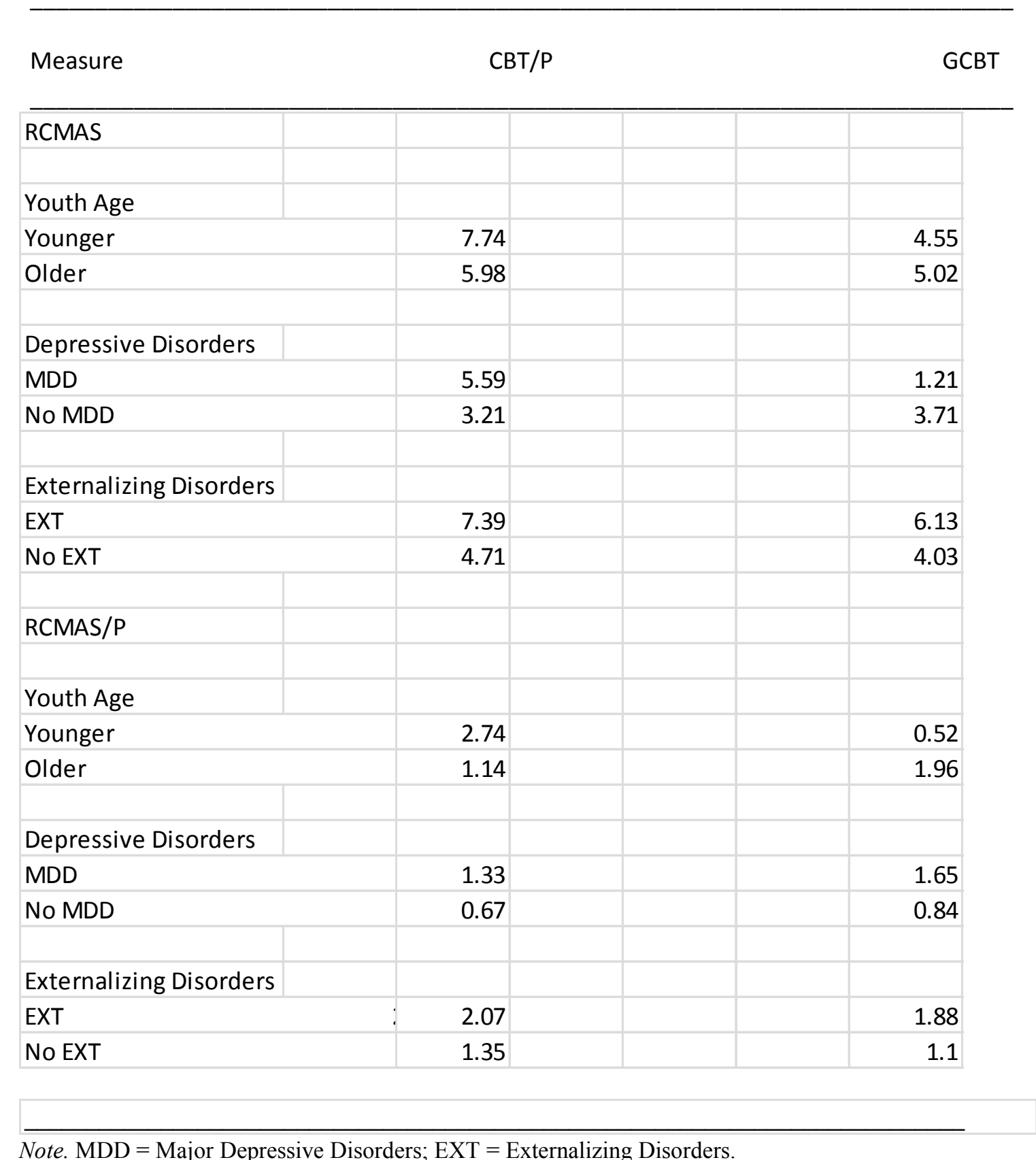


Table 5. Single Degree of Freedom Contrasts: Secondary Outcomes

\begin{tabular}{|c|c|c|c|c|c|c|}
\hline Contrast & Parameter & $S E$ & $t$ Value & $p$ Value & \multicolumn{2}{|c|}{$95 \% \mathrm{CI}$} \\
\hline \multicolumn{7}{|l|}{$C D I$} \\
\hline ME: Pre to Post & 19.09 & 3.94 & 4.85 & $<.001$ & 11.38 & 26.81 \\
\hline ME: Treatment & -0.84 & 0.08 & -1.02 & $>.05$ & -0.25 & 0.08 \\
\hline SME: Pre to Post for CBT/P & 16.85 & 4.8 & 3.51 & $<.001$ & 7.44 & 26.25 \\
\hline SME: Pre to Post for GCBT & 24.58 & 8.44 & 2.98 & $<.05$ & 8.43 & 40.73 \\
\hline ME: Post - FU & 19.98 & 4.74 & 4.74 & $<.001$ & 10.7 & 29.71 \\
\hline ME: Treatment & -0.08 & 0.07 & -1.14 & $>.05$ & -0.21 & 0.06 \\
\hline SME: Post-FU for CBT/P & 19.16 & 6.23 & 3.08 & $<.05$ & 6.96 & 31.67 \\
\hline SME: Post-FU for GCBT & 24.12 & 7.04 & 3.43 & 0.001 & 3.72 & 31.8 \\
\hline \multirow{2}{*}{\multicolumn{7}{|c|}{$C B C L-E$}} \\
\hline & & & & & & \\
\hline ME: Pre to Post & 64.9 & 7.86 & 8.24 & $<.001$ & 49.48 & 80.32 \\
\hline ME: Treatment & -1.2 & 1.36 & -0.87 & $>.05$ & -3.88 & 1.47 \\
\hline SME: Pre to Post for CBT/P & 39.01 & 9.59 & 4.06 & $<.001$ & 20.21 & 57.81 \\
\hline SME: Pre to Post for GCBT & 66.36 & 8.93 & 7.42 & $<.001$ & 48.84 & 83.88 \\
\hline ME: Post-FU & 60.2 & 11.47 & 5.24 & 0.001 & 37.72 & 82.69 \\
\hline ME: Treatment & -2.37 & 1.7 & -1.39 & $>.05$ & -5.7 & 0.95 \\
\hline SME: Post-FU for CBT/P & 25.17 & 1.79 & 1.79 & $<.05$ & -10.9 & 61.22 \\
\hline SME: Post-FU for GCBT & 43.25 & 2.8 & 2.8 & 0.01 & 12.99 & 73.51 \\
\hline
\end{tabular}

Note. $\mathrm{ME}=$ Main effects. SME = Simple Main Effects. CDI = Children's Depression Inventory. CBCL-E - Children's Behavior Checklist Externalizing Scale, CBT/P = Parent-involvement cognitive behavior treatment. GCBT $=$ Group cognitive behavior treatment. FU = Follow-Up. 
Table 6. Means for Variables at Pre, Post and Follow-Up

\begin{tabular}{lccc}
\hline Measure & Pretreatment & Posttreatment & Follow-Up \\
\hline RCMAS & 13.29 & $7.48^{\mathrm{a}}$ & $6.83^{\mathrm{b}}$ \\
RCMAS/P & 13.25 & $8.25^{\mathrm{a}}$ & $6.30^{\mathrm{b}}$ \\
MASC & 12.49 & $8.34^{\mathrm{a}}$ & $7.46^{\mathrm{b}}$ \\
CDI & 10.47 & $6.24^{\mathrm{a}}$ & $5.65^{\mathrm{b}}$ \\
CBCL-E & 53.17 & $47.51^{\mathrm{a}}$ & $46.31^{\mathrm{b}}$
\end{tabular}

Note. Posttreatment means with superscript "a" represent statistically significantly changes from pretreatment to posttreatment $(p<.05)$. Follow-up means with superscript " $b$ " represent statistically significantly changes from posttreatment to follow-up $(p<.05)$. RCMAS = Revised Children's Manifest Anxiety Scale, CDI = Children's Depression Inventory, CBCL-E - Children's Behavior Checklist Externalizing Scale, $\mathrm{P}=$ parent. 


\begin{tabular}{|c|c|c|c|c|c|}
\hline Model & Chi Square & CFI & RMSEA & $p$ Close & SRMR \\
\hline RCMAS and CDI & 10.6 & 0.99 & 0.02 & 0.91 & 0.01 \\
\hline CDI and RCMAS & 4.95 & 1 & $<.001$ & 0.77 & 0.01 \\
\hline MASC and CDI & 2.33 & 1 & $<.001$ & 0.69 & 0.01 \\
\hline CDI and MASC & 2.96 & 1 & $<.001$ & 0.93 & 0.01 \\
\hline RCMAS/P and CBCL-E & 12.81 & 1 & $<.001$ & 0.9 & 0.03 \\
\hline CBCL-E and RCMAS/P & 11.69 & 1 & $<.001$ & 0.8 & 0.03 \\
\hline \multicolumn{6}{|c|}{$\begin{array}{l}\text { Note. Chi square is goodness of fit index based on maximum likelihood criterion (model } \\
\text { degrees of freedom = 2), CFI is the Comparative Fit Index, RMSEA is the Root Mean } \\
\text { Square Error Approximation test and p Close is the p value for close fit associated with } \\
\text { the RMSEA, SRMR is the Square Root Mean Residual, RCMAS = Revised Children's } \\
\text { Manifest Anxiety Scale, CDI = Children's Depression Inventory, MASC = } \\
\text { Multidimensional Anxiety Scale for Children, CBCL-E - Children's Behavior Checklist } \\
\text { Externalizing Scale, P = parent. }\end{array}$} \\
\hline
\end{tabular}




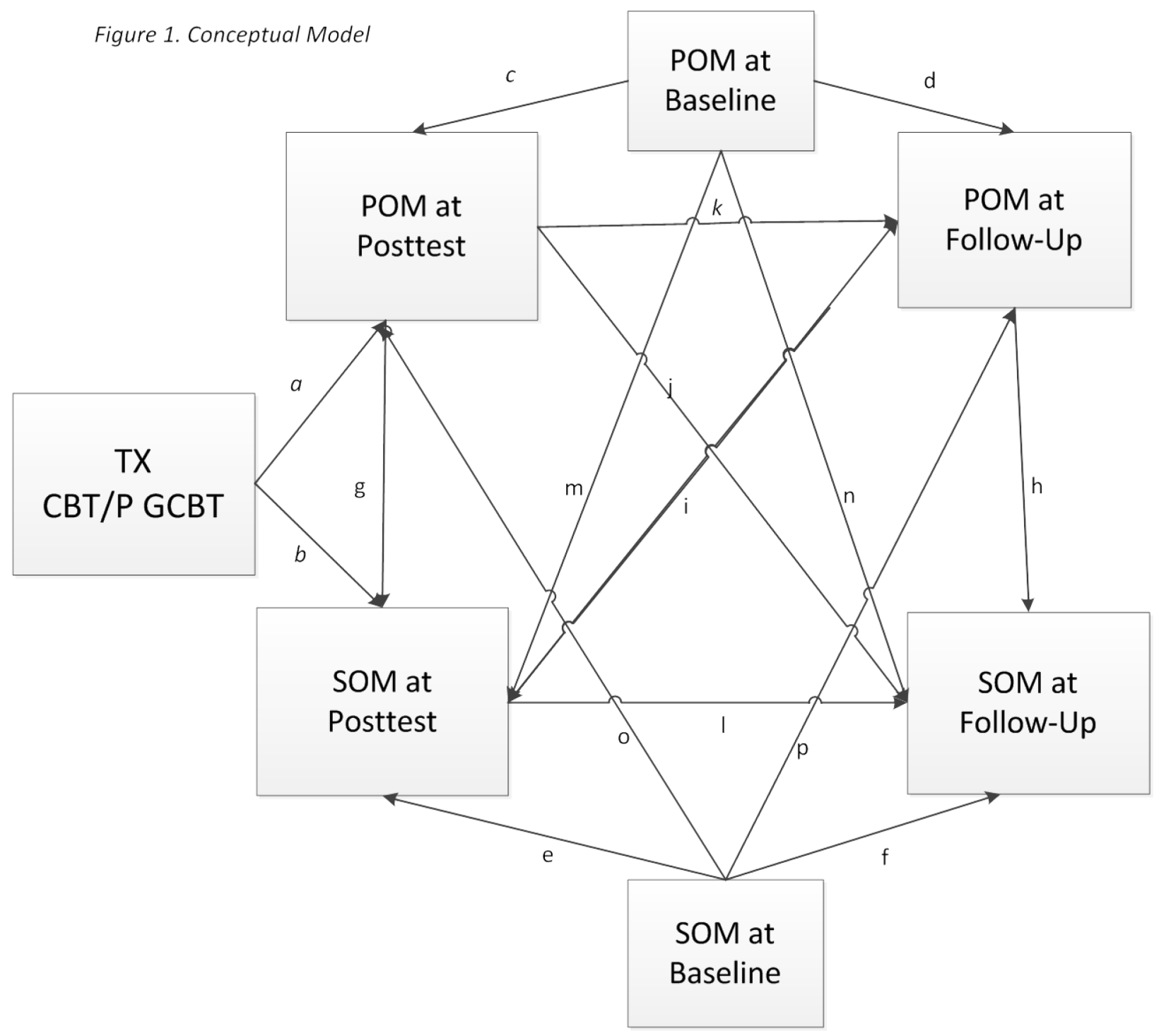

Note. $\mathrm{CBT} / \mathrm{P}=$ Parent-Involvement Cognitive Behavior Treatment. GCBT $=$ Group Cognitive Behavior Treatment. POM = Primary Outcome Measure; SOM = Secondary Outcome Measure. 


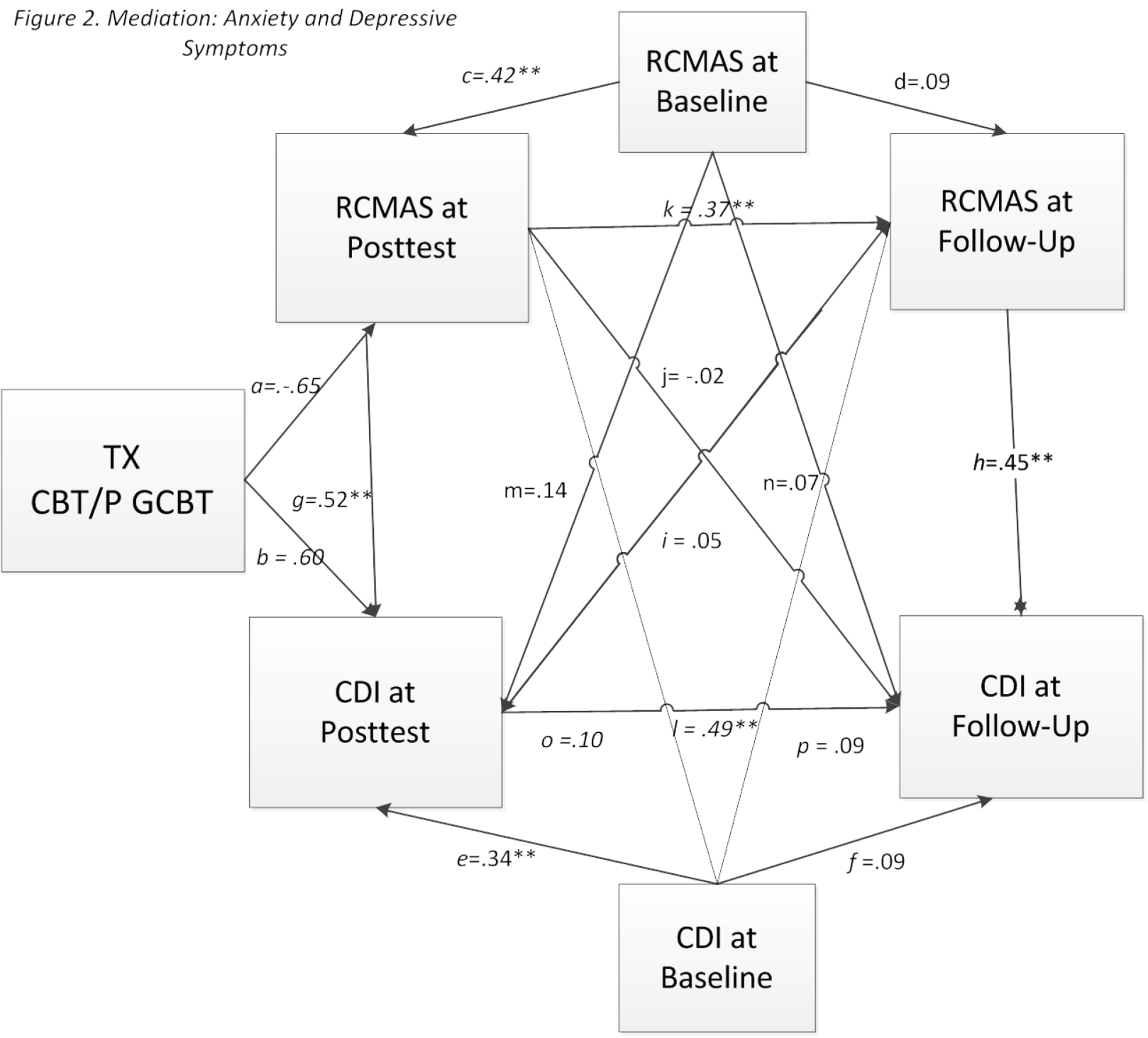

Note. $\mathrm{CBT} / \mathrm{P}=$ Parent-Involvement Cognitive Behavior Treatment. GCBT $=$ Group Cognitive Behavior Treatment. RCMAS $=$ Revised Children's Manifest Anxiety Scale, $\mathrm{CDI}=$ Children's Depression Inventory. $* *=p<.05$. 


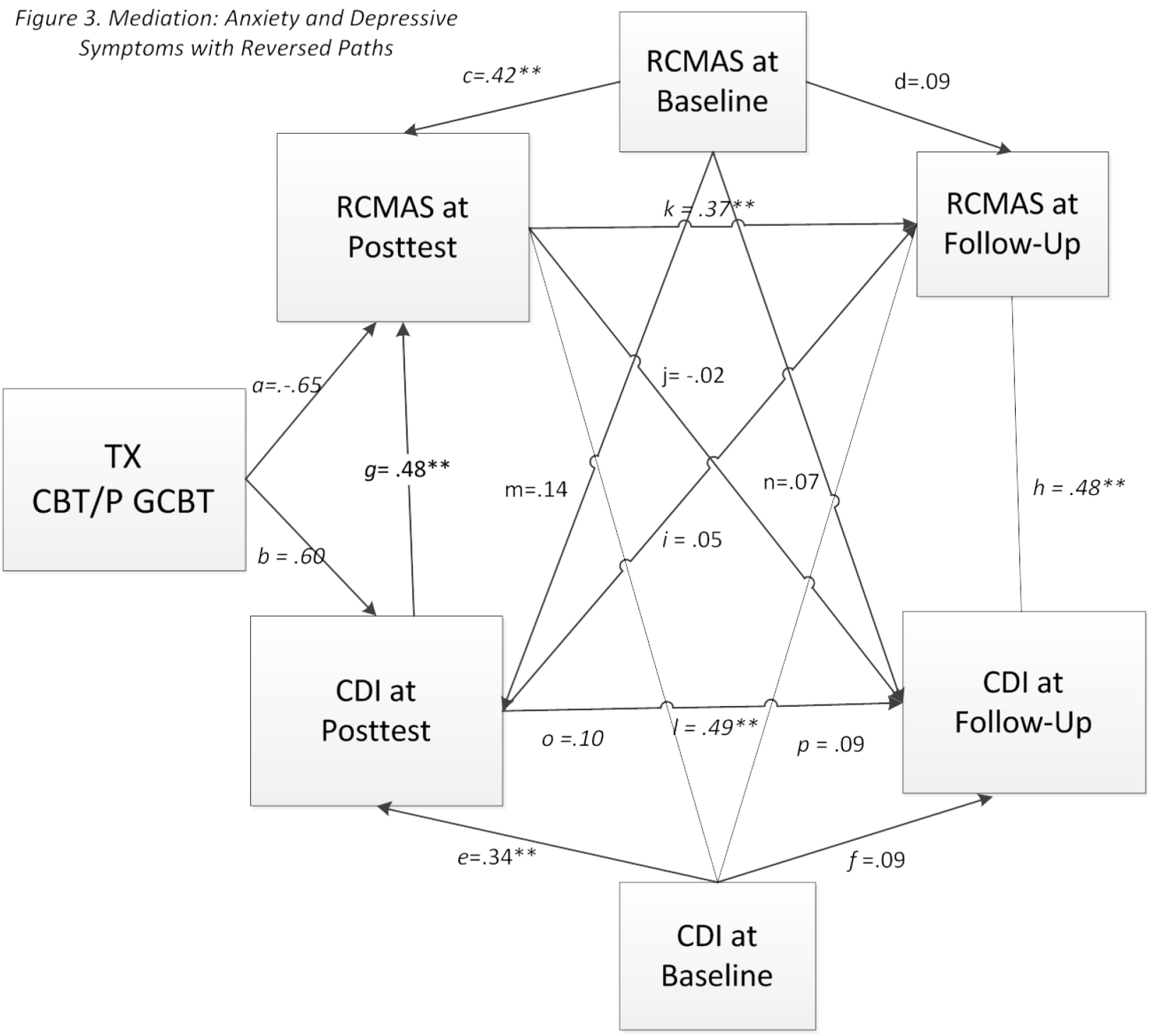

Note. $\mathrm{CBT} / \mathrm{P}=$ Parent-Involvement Cognitive Behavior Treatment. GCBT $=$ Group Cognitive Behavior Treatment. RCMAS = Revised Children's Manifest Anxiety Scale, $\mathrm{CDI}=$ Children's Depression Inventory. $* *=p<.05$. 


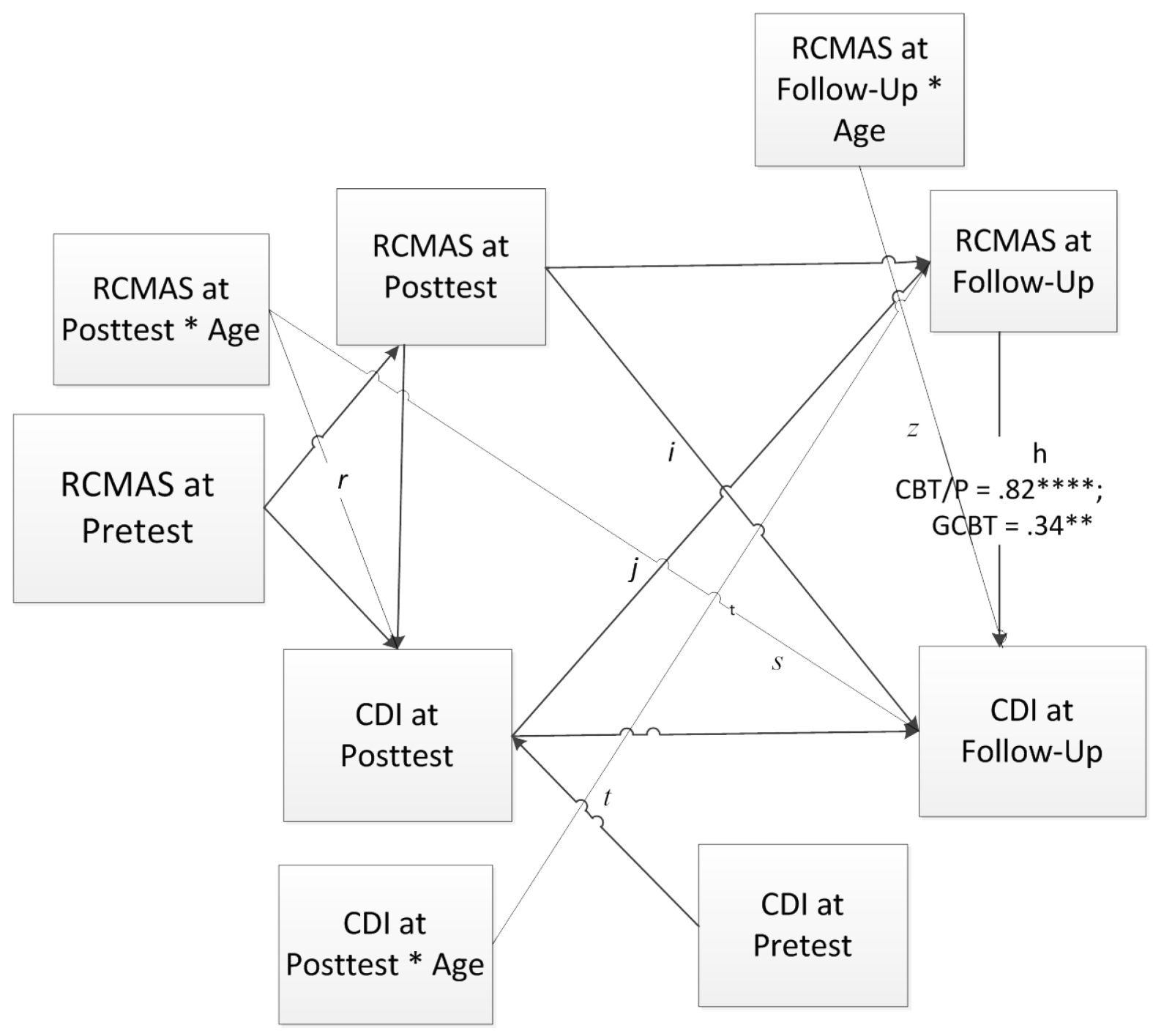

Figure 4. Moderated Mediation: Anxiety and Depressive Symptoms

Note. $\mathrm{CBT} / \mathrm{P}=$ Parent-Involvement Cognitive Behavior Treatment. GCBT $=$ Group Cognitive Behavior Treatment. RCMAS = Revised Children's Manifest Anxiety Scale, $\mathrm{CDI}=$ Children's Depression Inventory. Significant paths were moderated by treatment approach. Path coefficients for CBT/P are listed first followed by path coefficients for GCBT. $* *=p<.05 ; * * * *=$ significant interaction. 


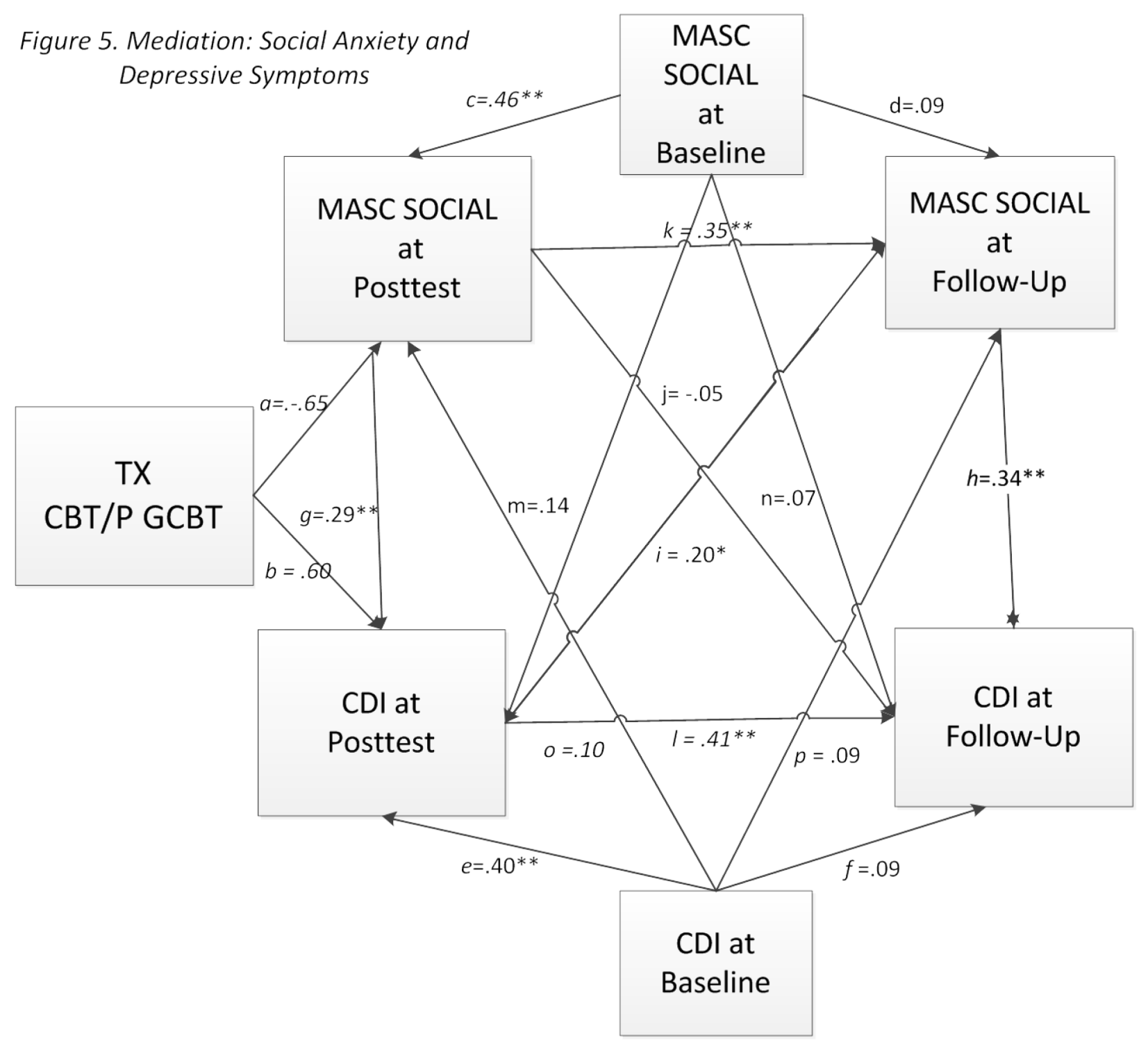

Note. $\mathrm{CBT} / \mathrm{P}=$ Parent-Involvement Cognitive Behavior Treatment. GCBT $=$ Group Cognitive Behavior Treatment. MASC $=$ Multidimensional Anxiety Scale for Children, $\mathrm{CDI}=$ Children's Depression Inventory. $* *=p<.05$. 


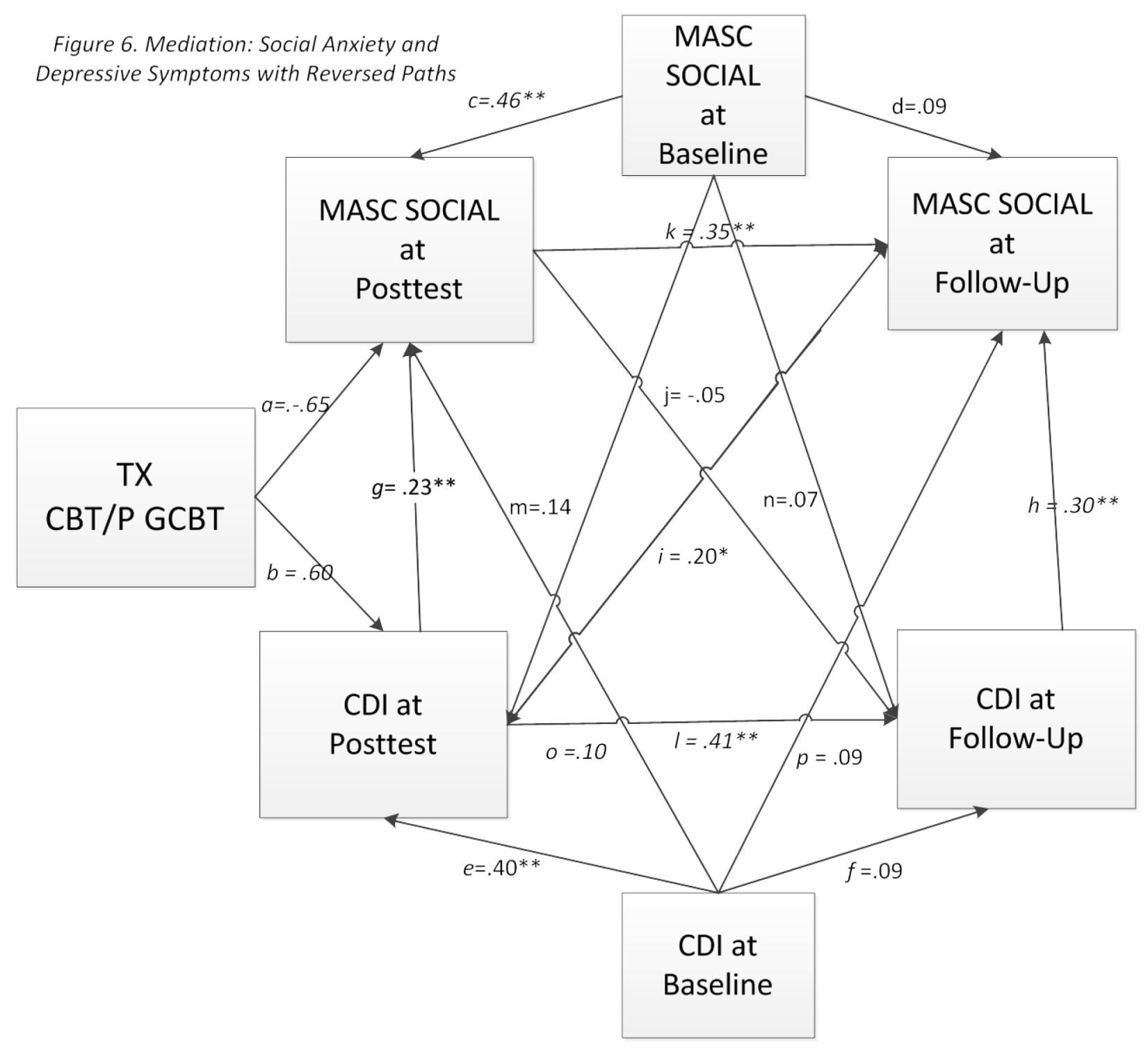

Note. $\mathrm{CBT} / \mathrm{P}=$ Parent-Involvement Cognitive Behavior Treatment. GCBT $=$ Group Cognitive Behavior Treatment. MASC $=$ Multidimensional Anxiety Scale for Children, $\mathrm{CDI}=$ Children's Depression Inventory. $* *=p<.05$ 


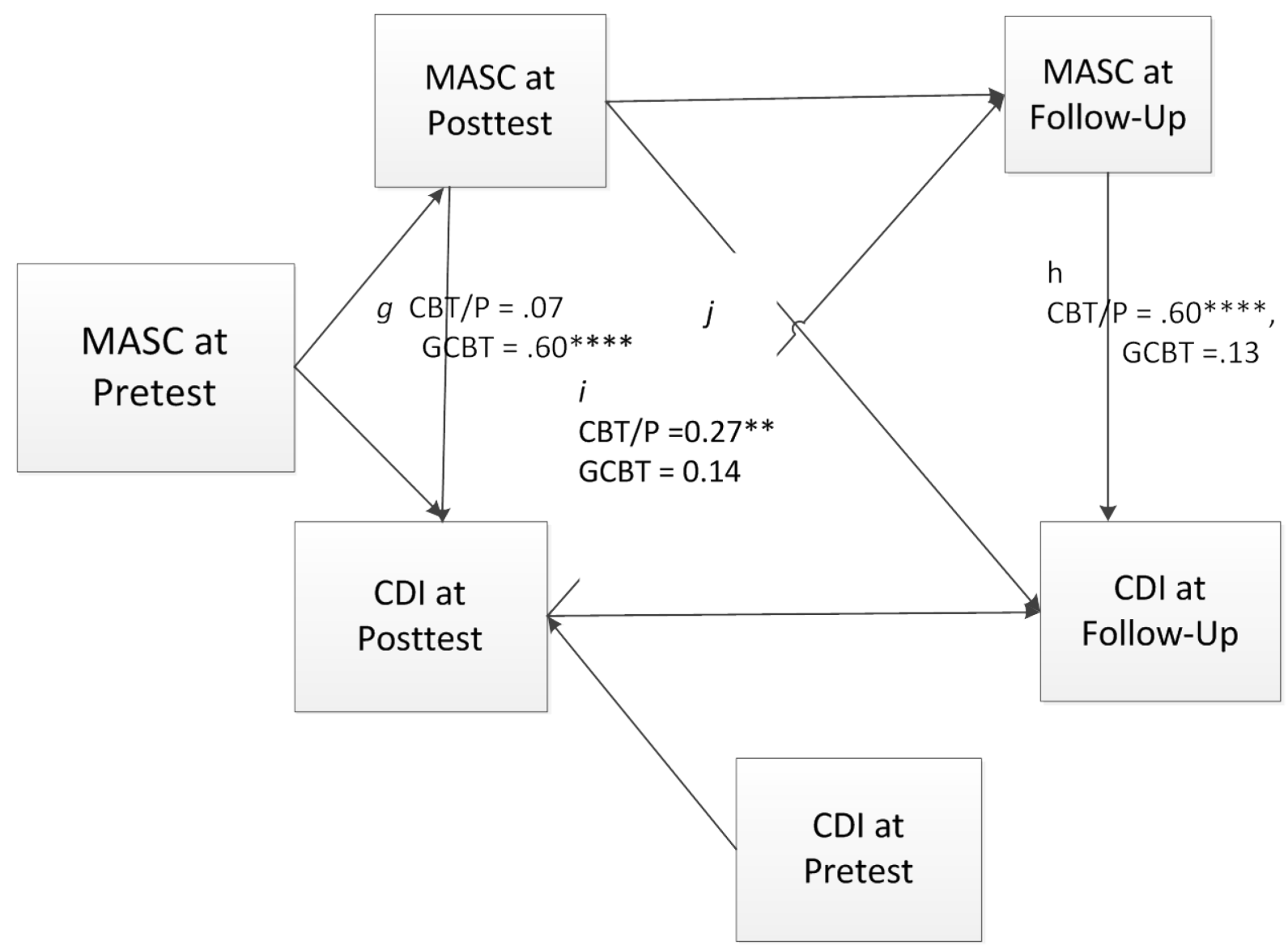

Figure 7. Moderated Mediation by Treatment: Social Anxiety and Depressive Symptoms

Note. $\mathrm{CBT} / \mathrm{P}=$ Parent-Involvement Cognitive Behavior Treatment. GCBT $=$ Group Cognitive Behavior Treatment. MASC $=$ Multidimensional Anxiety Scale for Children, $\mathrm{CDI}=$ Children's Depression Inventory. $*=p<.05 ; * * * *=$ significant interaction. 


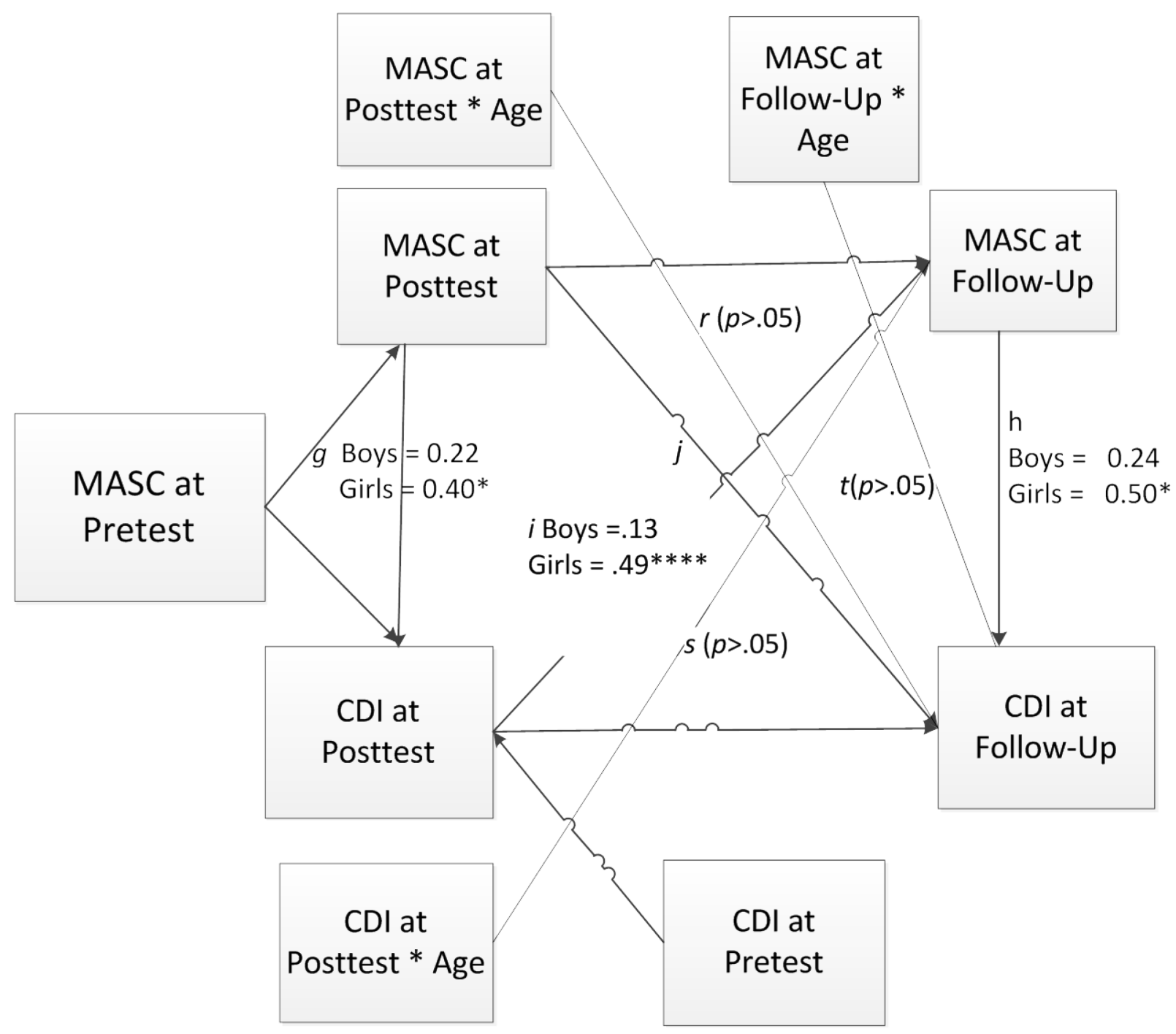

Figure 8. Moderated Mediation by Sex and Age: Social Anxiety and Depressive Symptoms

Note. $\mathrm{CBT} / \mathrm{P}=$ Parent-Involvement Cognitive Behavior Treatment. GCBT $=$ Group Cognitive Behavior Treatment. MASC $=$ Multidimensional Anxiety Scale for Children, $\mathrm{CDI}=$ Children's Depression Inventory. $* *=p<.05 ; * * * *=$ significant interaction. 


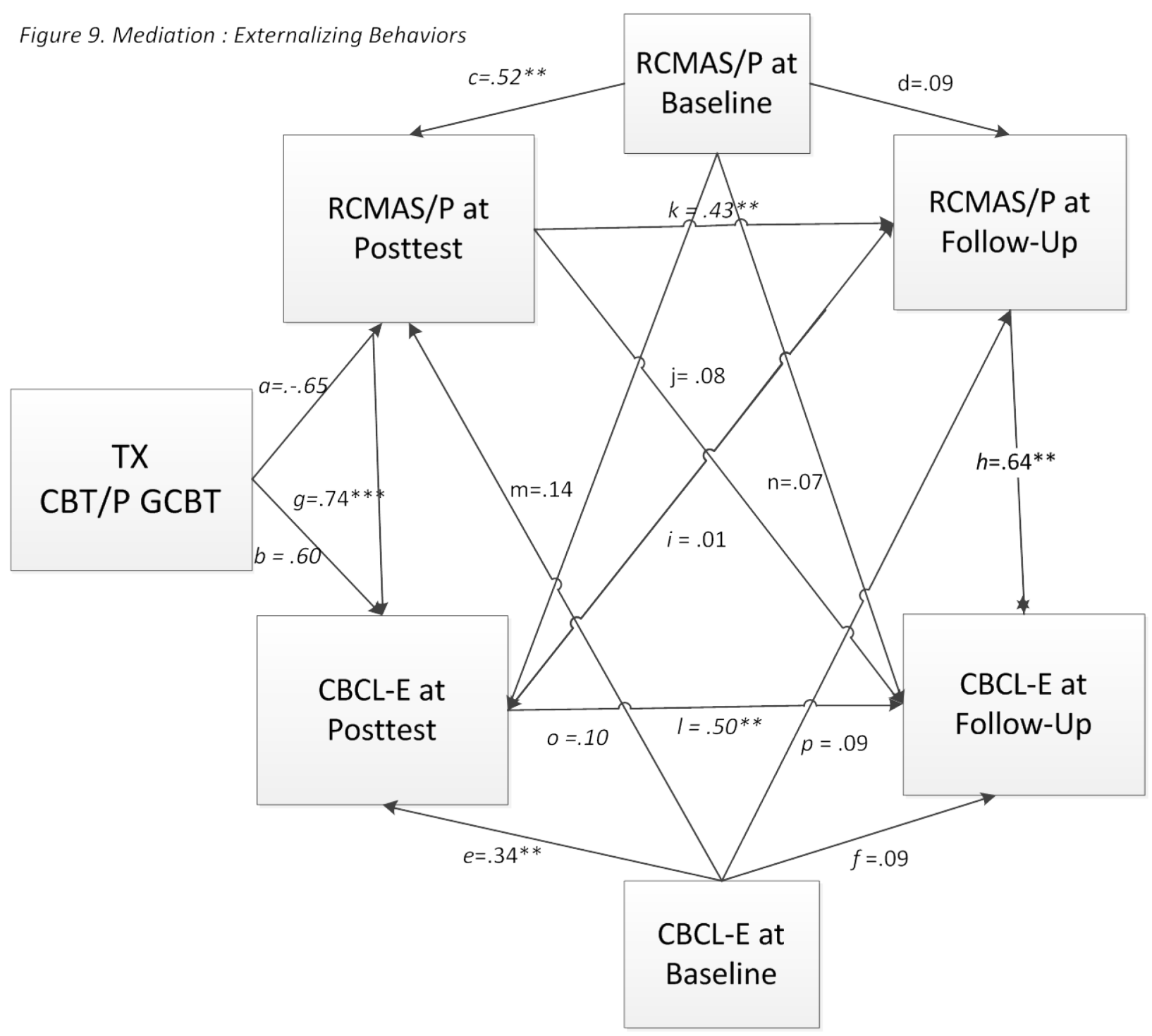

Note. $\mathrm{CBT} / \mathrm{P}=$ Parent-Involvement Cognitive Behavior Treatment. GCBT $=$ Group Cognitive Behavior Treatment. RCMAS = Revised Children's Manifest Anxiety Scale, CBCL-E - Children's Behavior Checklist Externalizing Scale, $\mathrm{P}=$ parent; $p<.05$. 


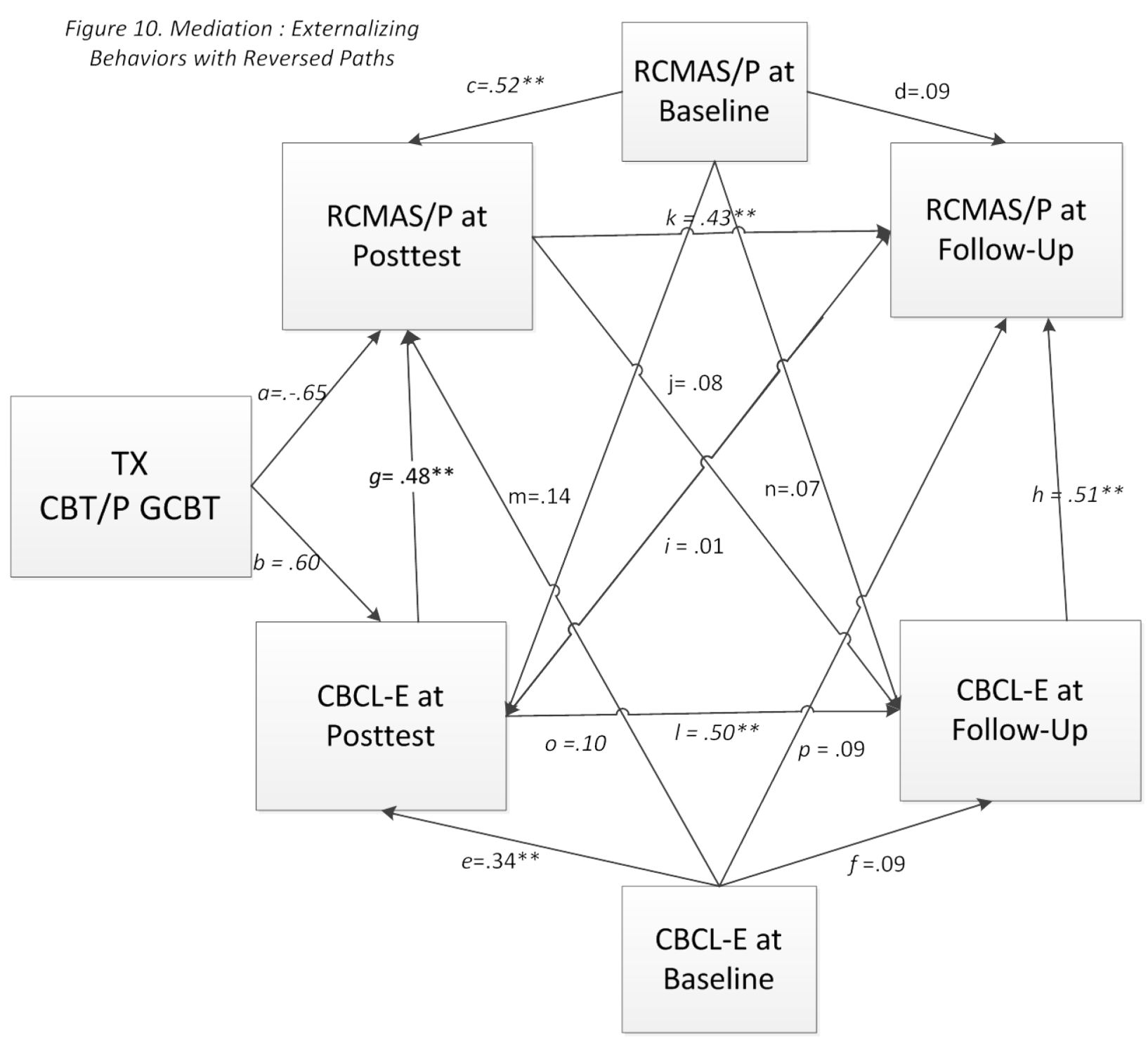

Note. $\mathrm{CBT} / \mathrm{P}=$ Parent-Involvement Cognitive Behavior Treatment. GCBT $=$ Group Cognitive Behavior Treatment. RCMAS = Revised Children's Manifest Anxiety Scale, CBCL-E - Children's Behavior Checklist Externalizing Scale, $\mathrm{P}=$ parent; $p<.05$. 


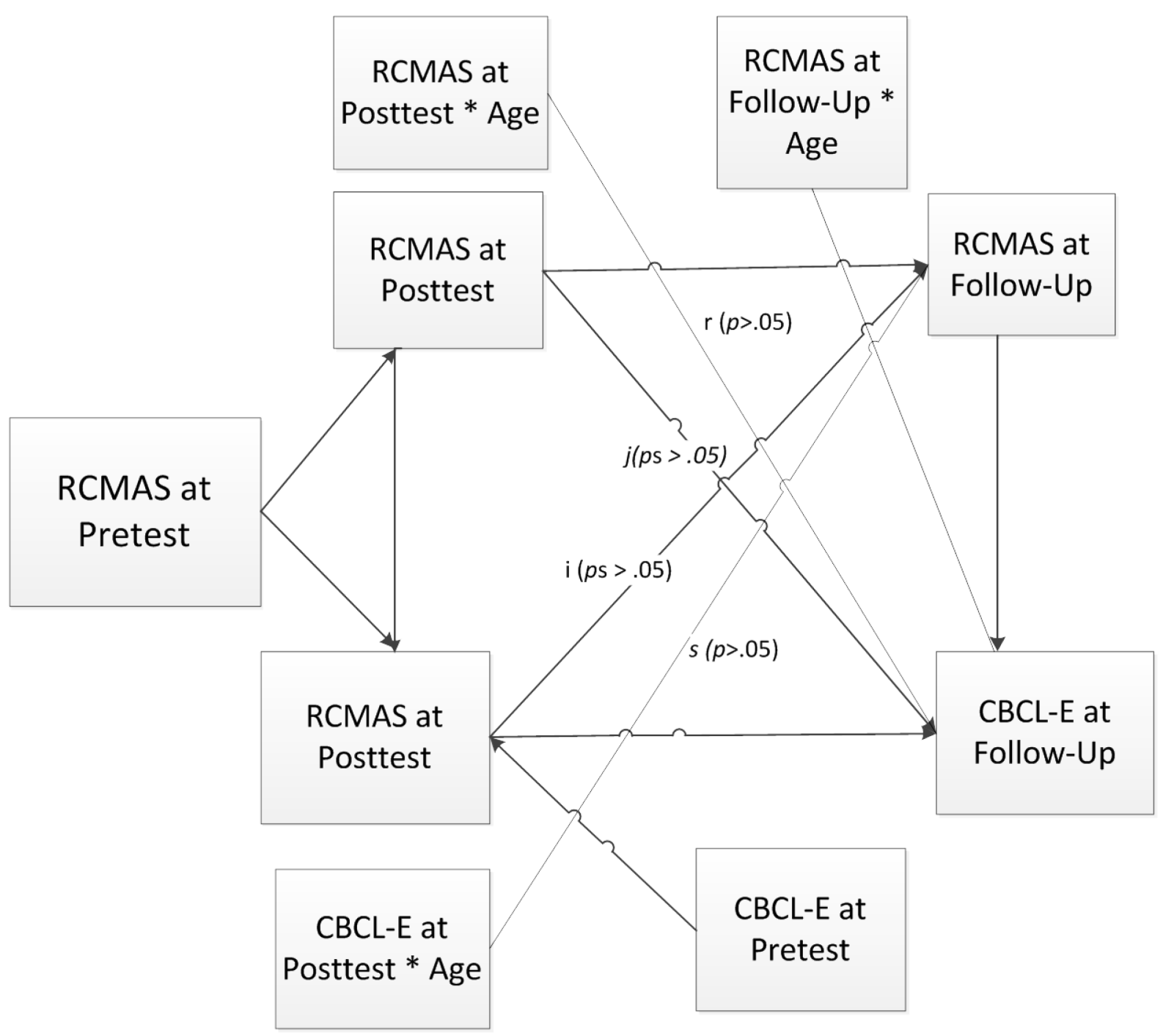

Figure 11. Moderated Mediation: Anxiety Symptoms and Externalizing Behaviors

Note. $\mathrm{CBT} / \mathrm{P}=$ Parent-Involvement Cognitive Behavior Treatment. GCBT $=$ Group Cognitive Behavior Treatment. RCMAS = Revised Children's Manifest Anxiety Scale, CBCL-E - Children's Behavior Checklist Externalizing Scale, P = parent. 


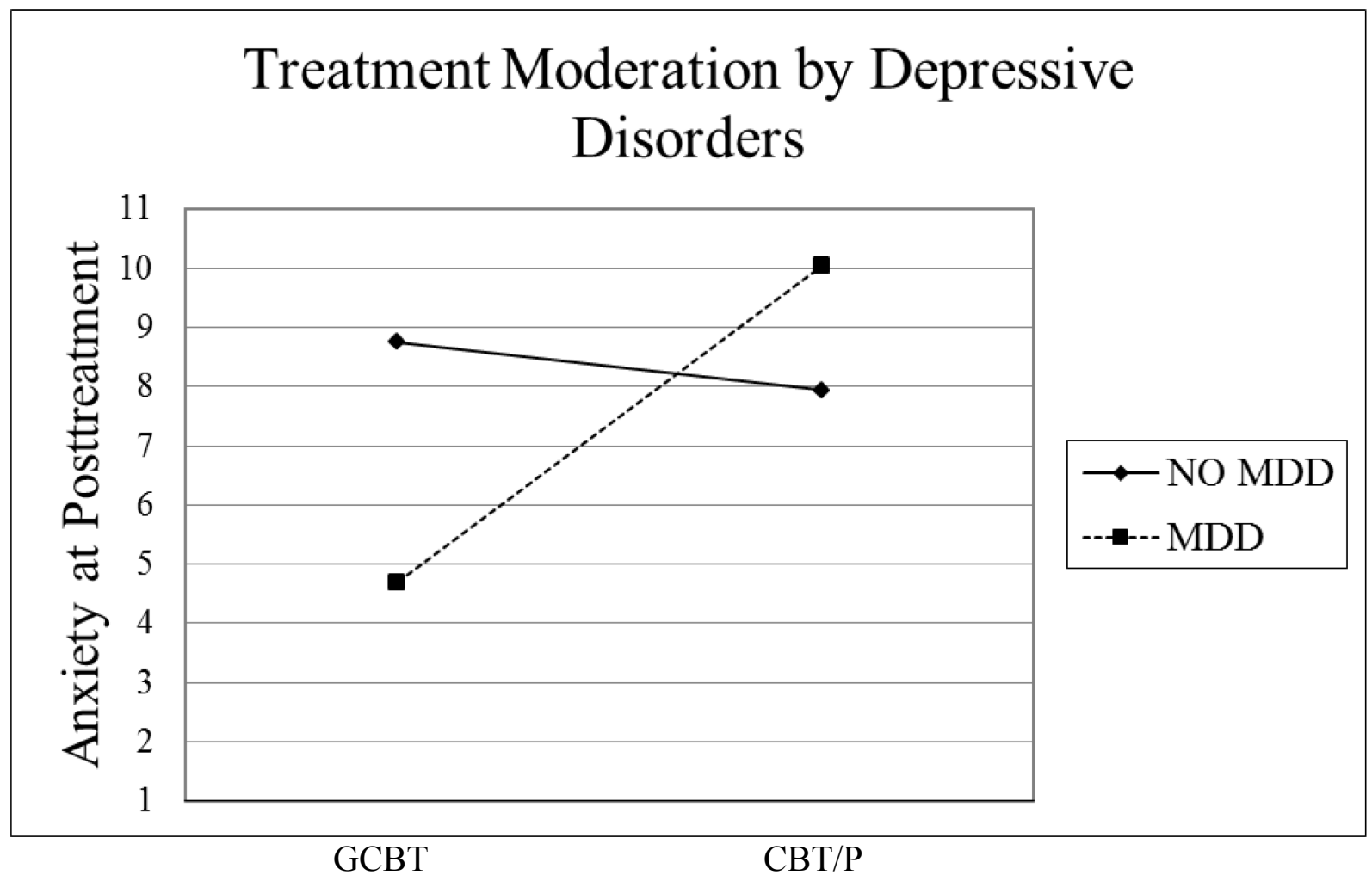

Figure 12. Treatment Moderation by Depressive Disorders 


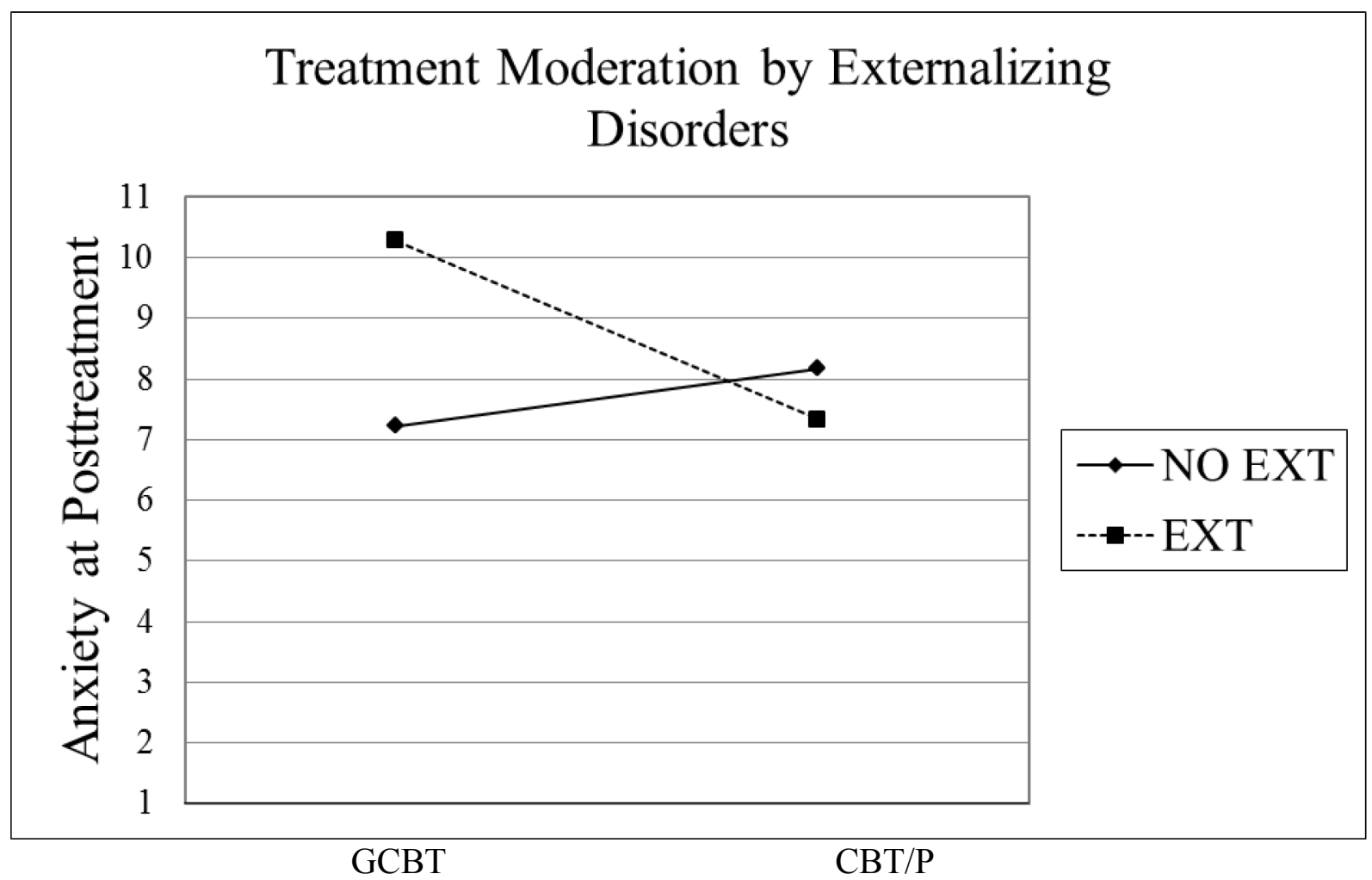

Figure 13. Treatment Moderation by Externalizing Disorders 


\section{LUCI M. MOTOCA}

2007

2009

2010 to Present

\author{
B.A., Psychology, Summa Cum Laudae \\ Temple University \\ Philadelphia, Pennsylvania \\ M.S., Developmental Psychology \\ Florida International University \\ Miami, Florida \\ Doctoral Candidate \\ Florida International University \\ Miami, Florida
}

\section{PUBLICATIONS}

Motoca, L. M., Williams, S., \& Silverman, W. K. (2012). Social Skills as a mediator between anxiety symptoms and peer interactions among children and adolescents. Journal of Clinical Child and Adolescent Psychology, 41, 329-336.

Silverman, W. K., \& Motoca, L. M. (2011). Treatment: Update and recommendations. In Silverman, W. K., \& Field, A. (Eds.). Anxiety disorders in children and adolescents: Research, assessment and intervention, pp. 392-418. Cambridge, U. K.: Cambridge University Press.

\section{PRESENTATIONS}

Motoca, L. M., Hernandez, I., Pienkowski, M., Dahan, J., \& Silverman, W. K. (2012, April). Using Behavior Observation Tasks to Assess Parenting Behaviors in a Clinic Referred Sample of Anxious Children and Adolescents. In J. Fox (Chair), Early Intervention of Internalizing Disorders: The Current State of Family-Based Approaches. Symposium conducted at the annual convention of the Anxiety Disorders Association of America (ADAA), Arlington, VA.

Motoca, L. M., Hernandez, I., Pienkowski, M., Dahan, J., \& Silverman, W. K. (2011, November). Using behavioral observation tasks to directly assess parenting behaviors in a clinic referred sample of children and adolescents. Poster presented at the annual meeting of the Association for Behavioral and Cognitive Therapies, Toronto, Canada. 
Marin, C. E., Motoca, L., M., Fredericks, I., Rey, Y., \& Silverman, W. K. (2011, November). The impact of academic transition and time of referral on symptoms severity and impairment in an anxious sample of youth. Poster presented at the annual meeting of the Association for Behavioral and Cognitive Therapies, Toronto, Canada.

Motoca, L. M., del Busto, C., Williams, S., \& Silverman, W. K. (2011, August). Clinical and social functioning of youth with and without social phobia. Poster presented at the annual meeting of the American Psychological Association, Washington, DC.

Motoca, L. M., Williams, S., \& Silverman, W. K. (2011, March). Anxiety symptoms, social skills, and peer interactions in clinic referred youth: A moderated mediation model. Poster presented at the annual meeting of the Anxiety Disorders Association of America. New Orleans, LA.

Motoca, L. M., Williams, S., Carter, R., \& Silverman, W. K. (2009, August). Predictors of treatment outcome in cognitive-behavioral therapy for anxious youth. Poster presented at the annual meeting of the American Psychological Association. Toronto, Canada.

Williams, S., Motoca, L. M., Saintil, M., Carter, R., \& Silverman, W. K. (2008, November). Child anxiety sensitivity and its relation to perceived parenting behaviors. Poster presented at the annual meeting of the Association for Behavioral and Cognitive Therapies, Orlando, FL. 